\title{
Infrared and Raman Studies of Charge Ordering in Organic Conductors, BEDT-TTF Salts with Quarter-Filled Bands
}

\section{Kyuya Yakushi}

Toyota Physical and Chemical Research Institute, 41-1, Yokomichi, Nagakute, Aichi 480-1192, Japan; E-Mail: yakushi@toyotariken.jp; Tel.: +81-561-57-9512; Fax: +81-561-63-6302

Received: 22 May 2012; in revised form: 4 July 2012 / Accepted: 20 July 2012 /

Published: 18 September 2012

\begin{abstract}
This paper reviews charge ordering in the organic conductors, $\beta$ "-(BEDT-TTF) (TCNQ), $\theta$-(BEDT-TTF $)_{2} \mathrm{X}$, and $\alpha$-(BEDT-TTF $)_{2} \mathrm{X}$. Here, BEDT-TTF and TCNQ represent bis(ethylenedithio)tetrathiafulvalene and 7,7,8,8-tetracyanoquinodimethane, respectively. These compounds, all of which have a quarter-filled band, were evaluated using infrared and Raman spectroscopy in addition to optical conductivity measurements. It was found that $\beta^{\prime \prime}$-(BEDT-TTF)(TCNQ) changes continuously from a uniform metal to a chargeordered metal with increasing temperature. Although charge disproportionation was clearly observed, long-range charge order is not realized. Among six $\theta$-type salts, four compounds with a narrow band show the metal-insulator transition. However, they maintain a large amplitude of charge order $(\Delta \rho \sim 0.6)$ in both metallic and insulating phases. In the $\mathrm{X}=\mathrm{CsZn}(\mathrm{SCN})_{4}$ salt with intermediate bandwidth, the amplitude of charge order is very small $(\Delta \rho<0.07)$ over the whole temperature range. However, fluctuation of charge order is indicated in the Raman spectrum and optical conductivity. No indication of the fluctuation of charge order is found in the wide band $X=I_{3}$ salt. In $\alpha$-(BEDT-TTF) ${ }_{2} I_{3}$ the amplitude of charge order changes discontinuously from small amplitude at high temperature to large amplitude $\left(\Delta \rho_{\max } \sim 0.6\right)$ at low temperature. The long-range chargeordered state shows ferroelectric polarization with fast optical response. The fluctuation of multiple stripes occurs in the high-temperature metallic phase. Among $\alpha$-(BEDT$\mathrm{TTF})_{2} \mathrm{MHg}(\mathrm{SCN})_{4}\left(\mathrm{X}=\mathrm{NH}_{4}, \mathrm{~K}, \mathrm{Rb}, \mathrm{Tl}\right)$, the fluctuation of charge order is indicated only in the $\mathrm{X}=\mathrm{NH}_{4}$ salt. $\alpha^{\prime}$-(BEDT-TTF) $)_{2} \mathrm{IBr}_{2}$ shows successive phase transitions to the ferroelectric state keeping a large amplitude of charge order $\left(\Delta \rho_{\max } \sim 0.8\right)$ over the whole temperature range. It was found that the amplitude and fluctuation of charge order in these compounds is enhanced as the kinetic energy (bandwidth) decreases.
\end{abstract}


Keywords: organic conductor; BEDT-TTF; charge order; infrared and Raman spectroscopy; vibrational spectroscopy; optical conductivity

\section{Introduction}

Vibrational spectroscopy is a powerful tool to detect the valence of molecules in organic charge-transfer salts. Among the normal modes of molecular vibrations, several vibrational modes change their eigenfrequencies depending on the valence of the molecule in question. These vibrational modes are called charge-sensitive modes. For example, the $\mathrm{C}=\mathrm{C}$ stretching mode is utilized as a probe for detecting the valence. As the chemical bond is usually much stronger than intermolecular interactions, the vibrational frequency and symmetry of a free molecule is approximately preserved in the solid state except in the case where a strong vibronic interaction occurs between a vibrational mode and a low-energy electronic state (electron molecular vibration coupling). A vibrational mode that strongly interacts with the electronic state is called structure-sensitive mode. Knowledge of the normal mode of a free molecule is essential, because a deviation from this mode in the solid state tells us which interaction is occurring. In this sense, reliable assignments of vibrational bands in the solid state are the most critical and indispensable. The charge-sensitive mode was applied for the first time to the distribution of charges in the unit cell of $\mathrm{Cs}_{2} \mathrm{TCNQ}_{3}$, where TCNQ represents 7,7,8,8-tetracyanoquinodimethane, wherein the infrared-active external ring $\mathrm{C}=\mathrm{C}$ stretching modes $\left(v_{4}\right)$ of $\mathrm{TCNQ}^{0}$ and $\mathrm{TCNQ}^{-}$were separately observed [1]. A detailed normal mode analysis of $\mathrm{TCNQ}^{0}$ and $\mathrm{TCNQ}^{-}$was reported by Bozio et al. [2]. Matsuzaki et al., extended the application to the TCNQ salt with fractional charge [3]. Subsequently, vibrational spectroscopy was applied to mixed-valence compounds of TCNQ [4], where one of the CN stretching modes of TCNQ was utilized as the probe.

The existence of Wigner-type charge order (CO) in organic conductors had been argued based on the finding of $4 \mathrm{k}_{\mathrm{F}}$ modulation in quasi-one-dimensional tetrathiafulvalene-7,7,8,8-tetracyanoquinodimethane (TTF-TCNQ) [5-8] because the wavelength of the $4 \mathrm{k}_{\mathrm{F}}$ modulation corresponds to the distance between localized electrons (holes) which are arranged with equal distance. However, the $4 k_{\mathrm{F}}$ modulation found in TTF-TCNQ was related to the $4 k_{\mathrm{F}}$ Peierls instability with on-site Coulomb repulsion. Since the amplitude of the charge density wave (CDW) is small, the charge-sensitive mode shows almost no linewidth broadening. The long-range $\mathrm{CO}$ of the Wigner-type was reported for quasi-one-dimensional (DI-DCNQI) ${ }_{2} \mathrm{Ag}$, where DI-DCNQI corresponds to 2,5-diiodo-dicyanoquinodiimine, by Hiraki and Kanoda using ${ }^{13} \mathrm{C}$-NMR [9]. They showed that the amplitude of CO $(\Delta \rho=0.5)$ is much larger than that of an incommensurate CDW. At about the same time, Seo theoretically predicted the $\mathrm{CO}$ ground state for $(\mathrm{TMTTF})_{2} \mathrm{X}(\mathrm{X}=\mathrm{Br}, \mathrm{SCN})$, where TMTTF corresponds to tetramethyltetrathiafulvalene, and (DIDCNQI $)_{2} \mathrm{Ag}$ using mean-field theory [10]. Later, Chow reported a similar charge order for $(\mathrm{TMTTF})_{2} \mathrm{X}\left(\mathrm{X}=\mathrm{PF}_{6}, \mathrm{AsF}_{6}\right)$ using ${ }^{13} \mathrm{C}-\mathrm{NMR}$ [11]. In the case of a two-dimensional organic compound, Moldenhauer et al., reported that localization of charge occurs in one of the stacks of $\alpha$ (BEDT-TTF) $)_{2} \mathrm{I}_{3}$ (BEDT-TTF corresponds to bis(ethylenedithio) tetrathiafulvalene) [12]. After the theoretical prediction of the $\mathrm{CO}$ ground state for $\alpha-(\mathrm{BEDT}-\mathrm{TTF})_{2} \mathrm{I}_{3}$ by Kino and Fukuyama [13], $\mathrm{CO}$ in two-dimensional organic compounds was suggested in $\alpha$-(BEDT-TTF $)_{2} \mathrm{I}_{3}$ [14], and more clearly shown 
in $\theta$-(BEDT-TTF $)_{2} \operatorname{RbZn}(\mathrm{SCN})_{4}$ [15]. Stimulated by the experimental findings of $\mathrm{CO}$, theoretical studies were conducted on the role of intersite Coulomb interaction in CO [16-18], lattice distortion accompanied by CO [19-21], the relationship between CO fluctuation and superconductivity (SC) [22], and quantum criticality at the edge of CO [23]. Subsequently, several experimental and theoretical studies have been conducted [24-27]. Some of these will be introduced from Section 3 on, in relation to the experimental results. Charge ordering continues to attract experimental and theoretical interest and attention. Some compounds in the CO state are regarded as unconventional ferroelectrics [28]. However, the ferroelectric properties have not been well investigated so far due to experimental difficulties. SC paring mediated by CO fluctuation was proposed by Merino and McKenzie [22]. Spectroscopic studies which may support the theoretical prediction for the SC mechanism have been reported [29-34]. It was also predicted that spin fluctuation mediates the SC pairing in a $\mathrm{CO}$ system [35]. Recently, it was pointed out that interaction with anions plays an essential role in the $\mathrm{CO}$ phase transition [36,37]. These subjects will be further investigated in future.

Along with NMR and x-ray diffraction, vibrational spectroscopy is a powerful tool to investigate the CO state, since the amplitude of CO is large. Raman spectroscopy was first applied to the charge ordering phase transition of $\theta-(\mathrm{BDT}-\mathrm{TTP})_{2} \mathrm{Cu}(\mathrm{NCS})_{2}$, for which the spectral features drastically changed [38,39]. Although the charge-sensitive mode is expected to show a simple splitting in a CO state, the Raman spectrum showed complicated splitting due to the additional splitting of the structure-sensitive mode. The fluctuation of charge order was obviously detected in the metallic phase. These characteristics were for the most part commonly observed in the compounds discussed here. In this paper, the author reviews infrared, Raman, and optical conductivity measurements of BEDT-TTF salts, which, apart from $\beta^{\prime \prime}$-(BEDT-TTF)(TCNQ), have a herringbone arrangement. This latter compound is included in this paper, because its unique behavior is necessary for understanding the metallic phase of $\theta$ - and $\alpha$-type BEDT-TTF salts.

The paper is organized as follows. Section 2 briefly describes the relation between the site charge and band structure, fluctuation of charge order and time correlation function, charge- and structure-sensitive modes of BEDT-TTF, electron-molecular vibration coupling, and the line shape when the site charge fluctuates. Section 3 describes charge disproportionation in $\beta$ "-(BEDT-TTF)(TCNQ), wherein a continuous change of the electronic state is discussed combined with optical conductivity. Section 4 describes the charge-ordering phase transition $\theta$-type BEDT-TTF salts; first, how the chargeand structure-sensitive modes split in the CO state; second, the metallic state with large CO amplitude is discussed; third, the metallic state with small CO amplitude is discussed. Section 5 introduces a spectroscopic investigation of $\alpha$-type BEDT-TTF. Here, the splitting of charge- and structure-sensitive modes in $\mathrm{CO}$ phase and ferroelectric properties are described first. Next, the splitting of the chargesensitive mode and fluctuation of $\mathrm{CO}$ in the metallic phase are discussed. Third, the non-uniform site charge distribution in the metallic phase under ambient pressure and hydrostatic pressure is compared with band calculations. Fourth, the optical conductivity is reviewed along with the collective $\mathrm{CO}$ excitation Finally, the successive ferroelectric transition of $\alpha^{\prime}-(\mathrm{BEDT}-\mathrm{TTF})_{2} \mathrm{IBr}_{2}$ is briefly described. The final section 6 presents the kinetic energy, which is associated with the bandwidth, of these compounds, and summarizes their electronic states correlated with kinetic energy. 


\section{Detection and Analysis of Charge Order}

\subsection{Site Charge and Fluctuation}

In solid organic charge-transfer salts, valence electrons are delocalized depending upon the magnitude of the overlap integral. Therefore, the valence of the molecule becomes a non-integer value, when the number of valence electrons does not equal the number of molecules. The fractional valence or fractional charge at the $j$ th site (site charge) in the unit cell, $\rho_{j}$, is given by the following equation:

$$
\rho_{j}=\sum_{k a, k b} \sum_{m}^{n_{M}}\left\langle k m\left|n_{j}\right| k m\right\rangle f(\varepsilon)
$$

where $|k m\rangle$ is the Bloch function of the $m$ th band, $n_{M}$ is the number of molecules in a unit cell, $n_{\mathrm{j}}$ is the number operator at the $j$ th site, and $f(\varepsilon)$ is the Fermi distribution function for a hole. The site charge is the integrated valence electron at each site below a Fermi level. Therefore, the site-charge distribution is sometimes non-uniform even in a metal, if the site in a unit cell is crystallographically non-equivalent. If the amplitude of charge order $\Delta \rho$ is small, a small portion of the valence electrons near the Fermi level contribute to the charge disproportionation, while in the case of large $\Delta \rho$, most valence electrons participate in the charge disproportionation as the band structure entirely changes

When the site charge dynamically fluctuates as $\rho(t)$, the correlation time $\tau$ is defined by the following equation,

$$
\tau=\int \frac{\left\langle\Delta \rho\left(t^{\prime}\right) \Delta \rho\left(t^{\prime}+t\right)\right\rangle}{\Delta^{2}} d t
$$

where $\Delta \rho\left(t^{\prime}\right)=\rho\left(t^{\prime}\right)-<\rho\left(t^{\prime}\right) \geq \rho\left(t^{\prime}\right)-1 / 2$ in the case of quarter filling, $<\Delta \rho\left(t^{\prime}\right) \Delta \rho\left(t^{\prime}+t\right)>$ is the time correlation function of $\rho$, and $\Delta^{2}=<\Delta \rho\left(t^{\prime}\right) \Delta \rho\left(t^{\prime}\right)>$ is the amplitude (variance) of fluctuation. As explained in the preceding paragraph, only the valence electrons near the Fermi level fluctuate, when the fluctuation amplitude is small. The charge fluctuation in a quarter-filled-band system was theoretically discussed in refs. [40,41] using the charge correlation function $\mathrm{C}(\boldsymbol{q}, \omega)$. If the system has CO instability, the charge correlation function has a strong peak at $\boldsymbol{q}_{0}$ and $\omega_{0} \sim 0$. The wave vector $\boldsymbol{q}_{0}$ specifies a charge-order pattern such as checkerboard or stripe. The peak frequency $\omega_{0}$ is the energy of the excited state which contributes to produce a $\mathrm{CO}$ wave in a uniform metal, and it may be related to the amplitude of $\mathrm{CO}$ fluctuation. The peak height and width are respectively associated with correlation length and correlation time. The time correlation function of the specified $\boldsymbol{q}_{0}$ is the Fourier transform of $\mathrm{C}\left(\boldsymbol{q}_{0}, \omega\right)$.

\subsection{Charge-Sensitive Mode of BEDT-TTF}

A normal mode analysis of BEDT-TTF ${ }^{0}$ and ${\mathrm{BEDT}-\mathrm{TTF}^{1+}}^{+}$was reported by Kozlov et al. $[42,43]$. The normal modes most sensitive to the charge (valence) of the BEDT-TTF molecule are the in-phase ( $v_{2}$, Raman active) and out-of-phase ( $v_{27}$ infrared active) stretching modes of the ring $\mathrm{C}=\mathrm{C}$ bonds, and the stretching mode of the $\mathrm{C}=\mathrm{C}$ bond bridging two five member rings ( $v_{3}$, Raman active). Kozlov et al., analyzed the normal mode assuming the $D_{2 h}$ symmetry, and their mode numbering is different from the subsequent numbering using more exact $D_{2}$ symmetry [44]. In this paper, Kozlov's numbering shown 
in Figure 1 will be used to avoid complication. In the solid state, site symmetry is usually lower than $\mathrm{D}_{2 \mathrm{~h}}$ or $\mathrm{D}_{2}$. However, the crystal field in organic conductors is much weaker than the covalent energy within a molecule. Therefore, the symmetry of the free molecule is approximately preserved in a crystal.

Figure 1. $C=C$ stretching modes of BEDT-TTF. The $v_{2}$ and $v_{27}$ modes are charge-sensitive, while the $v_{3}$ mode depends upon the network of transfer integrals, and thus it is structure-sensitive.
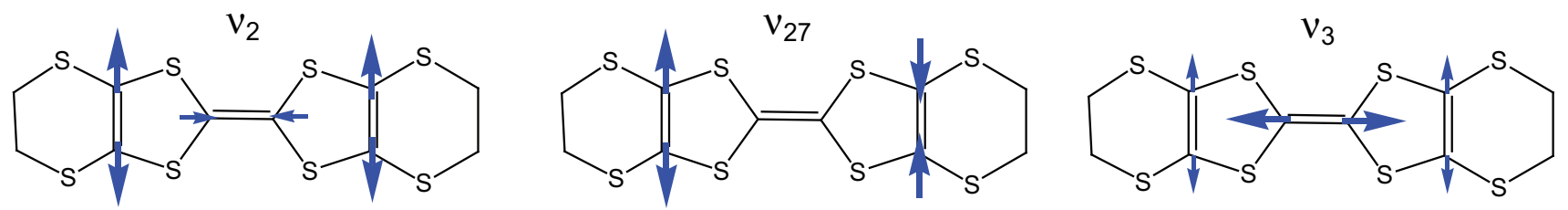

An attempt to examine the linear relationship between the frequency and fractional charge of BEDT-TTF was made by Wang et al., for the Raman-active $v_{2}$ and $v_{3}$ modes [45]. However, the frequency data were scattered about the linear relationship. In addition, they did not consider the effect of electron-molecular vibration interaction, which will be discussed in the next subsection. The linear relationship for the infrared-active $v_{27}$ was first utilized by Moldenhauer et al., for estimating the iconicity of BEDT-TTF [12]. However, this relationship is given based on the incorrect assignment of BEDT-TTF $^{+}$[46]. Subsequently, the relationship was not examined for long time, because this mode is usually hidden in a huge electronic absorption band when it is measured on the conducting plane or powdered samples. Recently, Yamamoto et al., have examined the assignment of Kozlov et al., and they reassigned $v_{2}, v_{3}$, and $v_{27}$ of $\mathrm{BEDT}_{-\mathrm{TTF}^{+}}$with the aid of ${ }^{13} \mathrm{C}$ and deuterium substituted compounds [46]. First they examined $v_{27}$ employing the polarization perpendicular to the conducting layer on the side face of single crystals. They found that the frequency of BEDT-TTF ${ }^{0}$ deviated from the linear relation, because it has a non-planar structure. They calculated the frequency of $v_{2}$ and $v_{27}$ assuming $D_{2}$ symmetry employing a DFT method, and found that the frequency of the flat structure BEDT-TTF ${ }^{0}$ satisfies the linear relationship. They presented an empirical relationship for the infrared active $v_{27}$ mode, $v_{27}(\rho)=1398+140(1-\rho)$. Next they examined the Raman-active $v_{2}$ and $v_{3}$ modes. The $v_{3}$ mode is more strongly perturbed by the electron-molecular vibration (EMV) interaction than the effect of charge. As we will explain in the next section, the highest-frequency $v_{3}$ mode does not shift, even if the charge disproportionation occurs, whereas the $v_{2}$ mode at charge-rich site $\left(v_{2 R}\right)$ shifts toward lower frequency. Consequently, the line $v_{2 R}(\rho)$ crosses the line $v_{3}(\rho)$, at $\rho \sim 0.9$ (see Figure 2). As the result of the interaction between $v_{2 R}$ and $v_{3}$, the frequency of $v_{2 R}$ deviates from the linear relationship. They presented the linear relationship for the infrared-active $v_{2}$ as $v_{2}(\rho)=1447+120(1-\rho)$, which is available only in the range of $0<\rho<0.8$. The $\rho$ dependence of the frequency is ascribed to the $\rho$ dependent force constant $F(\rho)$. That is, the linear relationship requires $(d F / d \rho)_{\rho=0}=(d F / d \rho)_{\rho=1}[47]$. However, the frequency of BEDT-TTF ${ }^{0.5+}$ deviates significantly from the linear relationship toward the low-frequency side, which means that $\left|(d F / d \rho)_{\rho=0}\right|>\left|(d F / d \rho)_{\rho=1}\right|$ (See Figure 7 of ref. [46]). Other nonlinear relationship at $\rho \sim 0.5$ will be discussed in Section 5.3.1. 
Figure 2. (a) Frequency of the vibronic modes plotted against the site charge of chargerich site $\left(\rho_{\mathrm{P}}+\rho_{\mathrm{R}}=1\right)$. The transfer integral is $t=0.2 \mathrm{eV}$, EMV coupling constants for $v_{2}$ and $v_{3}$ are $g_{2}=0.02 \mathrm{eV}$ and $\mathrm{g}_{3}=0.1 \mathrm{eV}$, respectively. The dashed line shows the sitecharge dependence when $\mathrm{g}_{2}=\mathrm{g}_{3}=0$, assuming a linear relationship against site charge $\rho$. The frequencies at $\rho=0$ and 1 are $v_{2}(0)=1567 \mathrm{~cm}^{-1}, v_{2}(1)=1447 \mathrm{~cm}^{-1}, v_{3}(0)=1520 \mathrm{~cm}^{-1}$, and $v_{3}(1)=1410 \mathrm{~cm}^{-1}$; (b) Contribution of the intermolecular excitation to the Raman tensor of the vibronic modes, which are plotted against the site charge of the charge-rich site; (c) Oscillator strength of the vibronic modes plotted against the site charge of the charge-rich site.
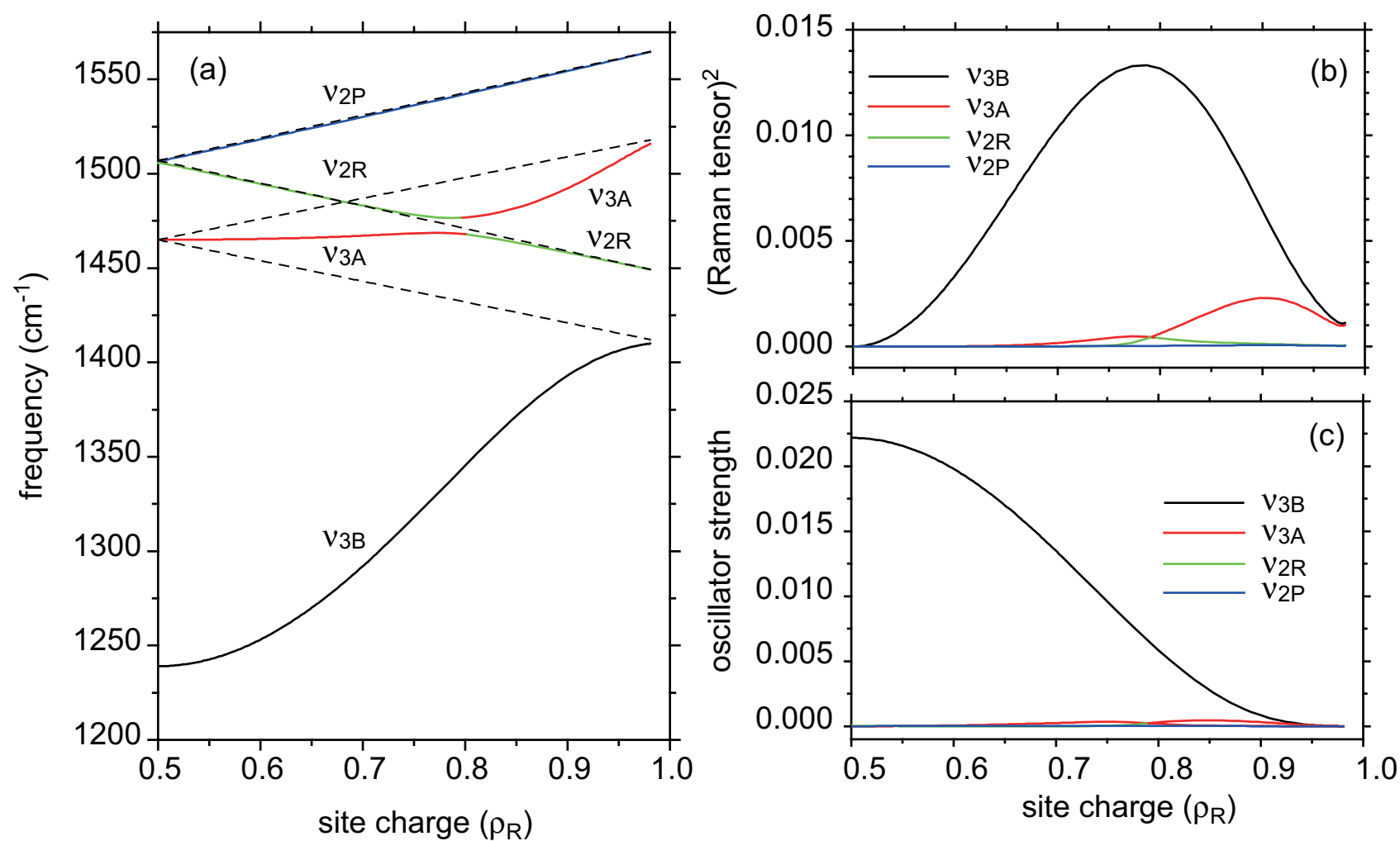

\subsection{Electron-Molecular Vibration (EMV) Coupling in BEDT-TTF}

The EMV interaction was first studied to interpret the strong infrared signal in the reflectivity polarized along the stacking axis in K-TCNQ [48]. Rice et al, , reported a symmetric dimer model and presented a comprehensive explanation for the EMV interaction [49]. According to the dimer model, the $a_{\mathrm{g}}$ mode of each molecule splits into in-phase and out-of-phase modes depending upon the magnitude of the coupling constant and transfer integral. The EMV interaction works only for the out-of-phase mode, and the coupling constant is estimated from the frequency difference between the Raman-active in-phase mode and the infrared-active out-of-phase mode. The EMV effect on the Raman-active mode was first pointed out by Girlando et al., in TTF-CA [50].

Based on the formulation by Painelli and Girlando [51], Yamamoto and Yakushi extended this idea to a disproportionate (asymmetric) dimer with average charge $\rho=0.5$, in which each site has a non-equivalent charge like $(B E D T-T T F)^{\rho+}(B E D T-T T F)^{(1-\rho)+}$ [52]. Figure 2 shows the numerical calculation of the frequency and intensity of vibronic modes plotted as a function of the site charge of 
the charge-rich site $(\rho>0.5)$. In this calculation, the transfer integral between two molecules is taken as $t=0.2 \mathrm{eV}$, and the coupling constants of $v_{2}$ and $v_{3}$ mode are taken as $g_{2}=0.02 \mathrm{eV}$ and $g_{3}=0.1 \mathrm{eV}$. $v_{2 \mathrm{P}}$ and $v_{2 \mathrm{R}}$, denote the $v_{2}$ mode of the charge-poor and charge-rich site (molecule), respectively, and $v_{3 \mathrm{~A}}$, and $v_{3 \mathrm{~B}}$ denote the $v_{3}$ mode for the in-phase and out-of-phase oscillation, respectively. The coupling constant in BEDT-TTF is estimated in refs. $[43,53]$. The coupling constants of $v_{2}$ and $v_{3}$ were respectively estimated to be $g_{2}=0.043$ and $g_{3}=0.071 \mathrm{eV}$ from an analysis of the infrared spectrum of the isolated dimer (BEDT-TTF $)_{2}{ }^{2+}[53]$. The parameters estimated from the infrared spectra were very scattered $\left(g_{2} \sim 0.007-0.039 \mathrm{eV}\right.$ and $g_{3}=0.007-0.081 \mathrm{eV}$ ) depending upon the compounds [54]. A more recent estimation gives $g_{3}=0.076 \mathrm{eV}$ from the analysis of the infrared spectrum of $\kappa-(\mathrm{BEDT}-\mathrm{TTF})_{2} \mathrm{Cu}\left[\mathrm{N}(\mathrm{CN})_{2}\right]-\mathrm{Br}_{0.85} \mathrm{Cl}_{0.15}$ [55]. Using the same data, Girlando interpreted this as $g_{2}=0.075 \mathrm{eV}$ [56]. The estimation of the coupling constants of $v_{2}$ and $v_{3}$ from the infrared spectrum seems to be unfixed still now. The coupling constants used for the model calculation (Figure 2) are qualitatively consistent with ref. [55] and with the interpretation of the Raman spectra of various compounds appearing in this paper.

As shown in Figure 2a, the weakly coupled modes, $v_{2 P}$ and $v_{2 R}$, approximately conform to a linear relationship. However, the strongly coupled modes, $v_{3 \mathrm{~A}}$ and $v_{3 \mathrm{~B}}$, deviate remarkably from the linear relation, although the frequency of $v_{3}$ of the free molecule follows a linear relationship as shown by the dashed lines in Figure 2a [56]. The $v_{3 \mathrm{~A}}$ and $v_{3 \mathrm{~B}}$ modes are respectively the in-phase and out-of-phase vibrations at $\rho=0.5$. The noteworthy point here is that the $v_{3 \mathrm{~A}}$ mode that is Raman-active keeps the frequency corresponding to $\rho=0.5$ in the range of $0.2<\rho<0.8$. This is because the in-phase vibrations at two sites are not perturbed by the EMV interaction [49]. Actually, the frequencies of $v_{3}$ of the 2:1 salts are nearly equal to those of the 3:2 salts in the work by Wang et al. [45] although they claim a linear relationship for $v_{3}$ in their paper. Near $\rho \sim 0.8$, mixing between $v_{2 R}$ and $v_{3 \mathrm{~A}}$ occurs, and the assignment becomes meaningless. This situation is sometimes seen in the charge-ordered state (See Sections 4.1, 5.1, and 5.3.3). This result means that Raman spectroscopy cannot determine the fractional charge when the molecular vibrations strongly interact with each other through the EMV mechanism. Fortunately, in the case of BEDT-TTF, the $v_{2}$ mode is available to determine the fractional charge, because the EMV coupling is weak enough. However, this mode is available only in the range of $0<\rho<0.8$. In this sense, the infrared-active mode, for example, $v_{27}$ of BEDT-TTF is the best probe to determine the site charge in a charge-ordered system. From the viewpoint of experimental technique, it is extremely difficult to observe $v_{27}$ in the conducting plane, because the phonon mode is screened by the strong electronic absorption band. Usually the crystal face parallel to a conducting plane is most developed. Therefore, the best way to detect $v_{27}$ is to measure the reflectivity on the thin side face of the crystal with the polarization perpendicular to the conducting plane.

In the infrared spectrum, we can observe only the out-of-phase mode $v_{3 B}$, which shows a large downshift due to the large EMV coupling constant, as shown in Figure 2c. As the out-of-phase mode dynamically modulates the orbital energy of the highest occupied molecular orbital (HOMO) of BEDT-TTF in the dimer, it transfers charge back and forth inducing an oscillating dipole along the direction connecting the centers of the molecules [49]. Accordingly, the out-of-phase $v_{3}$ mode is usually found very strongly in the optical conductivity of the conducting plane (See Sections 4 and 5). The downshift of this mode is sensitive to the transfer integrals between BEDT-TTF molecules (see ref. [54] for example). Yamamoto et al., discussed the relation between the downshift of $v_{3 \mathrm{~B}}$ and 
transfer integrals comparing the $v_{3}$ modes among several $\theta$-type of BEDT-TTF salts. [57] Utilizing this property, the symmetry can be determined, for example, by the presence of a glide plane (See Section 4.1) and breaking of inversion symmetry (See Section 5.1). The Raman intensity of the same vibronic mode $v_{3 B}$ is calculated to be strong in the disproportionate dimer (See Figure $2 b$ ). In this model, only the intermolecular excitation is considered for the calculation of the Raman tensor in the off-resonance condition. Actually, intramolecular excitations contribute more to the Raman tensor, and the resonance effect is sometimes quite large in the charge-transfer salts of BEDT-TTF. This calculation shows the additional contribution of the intermolecular excitation to the Raman tensor. As shown in Figure $2 b$, the contribution of this intermolecular excitation is largest in the vibronic $v_{3}$ mode that has a large EMV coupling constant. Actually, the vibronic $v_{3}$ mode is frequently found in the Raman spectrum of charge-ordered system (See Sections 4 and 5).

\subsection{The Line Shape of Charge-Sensitive Mode}

The frequency of the charge-sensitive mode strongly depends upon the site charge, for example, $\Delta \omega / \Delta \rho \approx 140 \mathrm{~cm}^{-1} / e$ or $\Delta \omega / \Delta \rho \approx-140 \mathrm{~cm}^{-1} / h$ for the $v_{27}$ mode. Let us consider a system in which the unit cell involves two sites (molecules) and one hole. If every site is equivalent, then every site has a uniform site charge (hole) $\rho=0.5$. When the system undergoes a charge-ordering transition, the site charge is disproportioned into $\rho_{\mathrm{R}}$ and $\rho_{\mathrm{P}}$. Therefore, the charge-sensitive mode splits into two vibrational bands from a single band. If the site charge fluctuates around $\rho=1 / 2$, the frequency also fluctuates around $v(\rho)$, and the linewidth is broadened depending on the fluctuation rate. This relation is illustrated in Figure 3.

Figure 3. Linear relationship between the site charge and frequency of $v_{27}$ [46]. The schematic sketch of the spectrum shows that the charge-sensitive mode of uniform site charge (green) is split into two (blue) by charge disproportionation, and the linewidth is broadened by the fluctuation of the site charge.

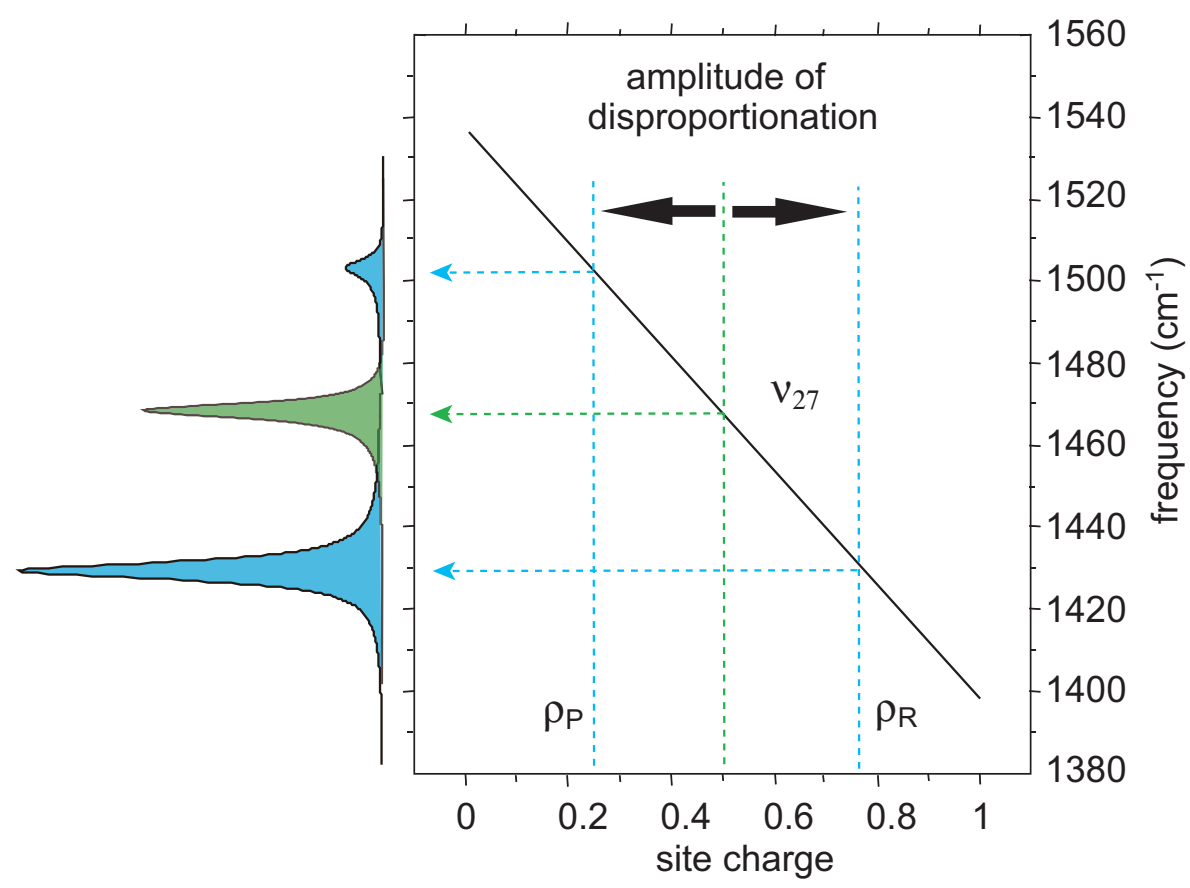


Figure 4. (a) Two-states-jump model calculated by Equation (3) with $\omega_{1 / 2}=0, \Gamma / \Delta=0.04$, $a_{\mathrm{R}}=10$, and $a_{\mathrm{P}}=0.3$; (b) Gaussian model calculated using Equation (4). $\tau$ and $\Delta$ are the correlation time and amplitude of fluctuation, respectively.
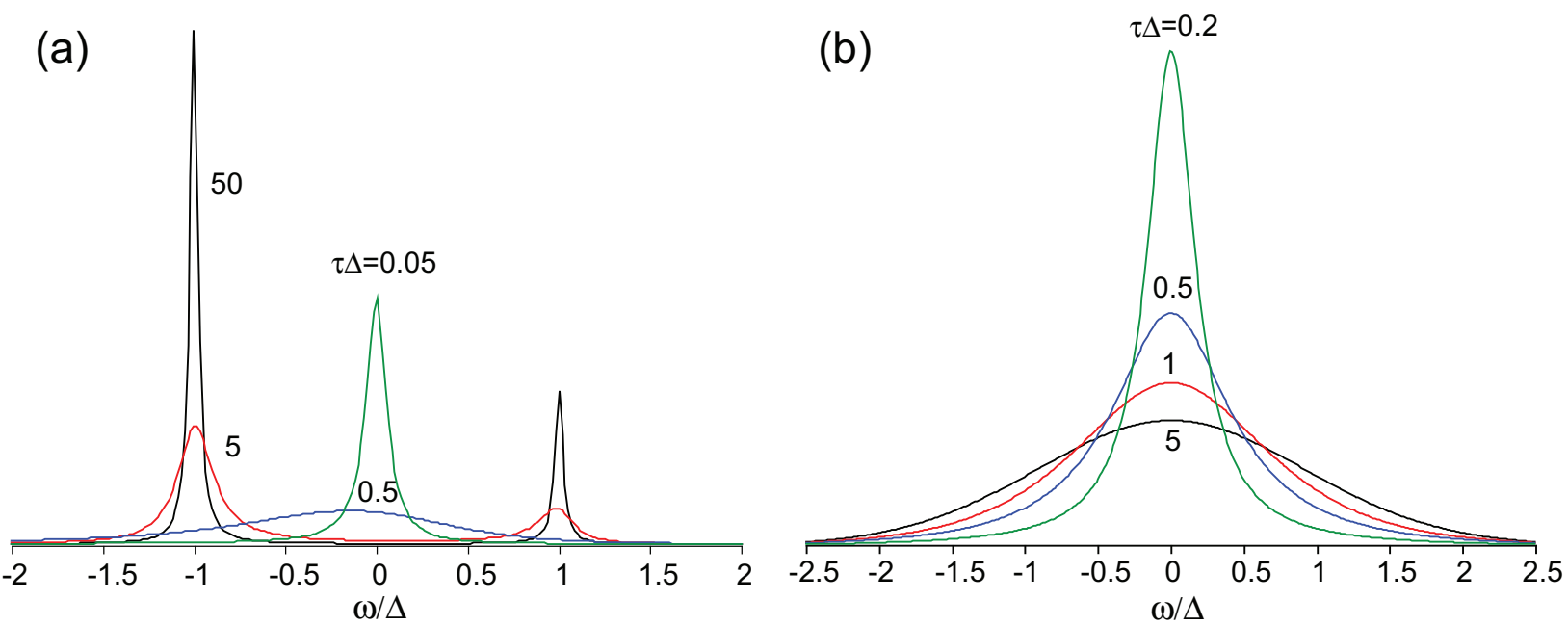

The fluctuation of the site charge is characterized by its amplitude (or variance $\Delta_{c}=\left\langle\Delta \rho^{2}\right\rangle^{1 / 2}$ ) and correlation time $\tau=\int_{0}^{\infty}\left(\langle\Delta \rho(0) \Delta \rho(t)\rangle / \Delta_{c}{ }^{2} d t\right.$, where $\Delta \rho=\rho(\mathrm{t})-1 / 2$ is the time-dependent site-charge variation from the average site charge $1 / 2$. This charge fluctuation modulates the frequencies of $v_{27}$ and $v_{2}$ with an amplitude $\Delta_{f}$ and correlation time $\tau$. When the site charge fluctuates stochastically between two values such as $\rho_{R}$ and $\rho_{P}$ and thus the frequency fluctuates between $\omega_{R}$ and $\omega_{P}$ with a transition rate $\gamma$, then the line shape of the charge-sensitive mode is described by the following equation [58].

$$
\left.I(\omega) \propto \operatorname{Re}\left[\begin{array}{ll}
a_{R} & a_{P}
\end{array}\right)\left(\begin{array}{cc}
\left(i \left(\omega-\left(\omega_{1 / 2}-\Delta\right)+\gamma / 2+\Gamma / 2\right.\right. & -\gamma / 2 \\
-\gamma / 2 & i\left(\omega-\left(\omega_{1 / 2}+\Delta\right)+\gamma / 2+\Gamma / 2\right.
\end{array}\right)^{-1}\left(\begin{array}{l}
a_{R} \\
a_{P}
\end{array}\right)\right]
$$

here $\omega_{1 / 2}$ is the frequency for $\rho=1 / 2, \Delta=\left(\omega_{\mathrm{P}}-\omega_{\mathrm{R}}\right) / 2, \Gamma$ is the natural width in the solid state, and $a_{\mathrm{R}}$ and $a_{\mathrm{P}}$ are parameters proportional to the transition dipole moment or Raman tensor of the charge-sensitive mode at charge-rich and charge-poor sites, respectively. In this case, the correlation time is equal to the staying time at a site which is given by $\tau=1 / \gamma$. When $\tau \Delta<<1$ or $\tau \Delta>>1$, the lineshape approaches a Lorentzian.

When the site charge fluctuates stochastically as a Gaussian process, the correlation function has an exponential decay as, $\langle\Delta \rho(0) \Delta \rho(t)\rangle=\Delta_{c}^{2} \exp (-\gamma t)$, where $\Delta_{c}$ is the amplitude of the fluctuation and $\gamma$ is the decay rate. In this case, the modulation of the site charge is transformed to a modulation of the frequency of the charge-sensitive mode, and the line shape of the charge-sensitive mode is given by the following equation $[59,60]$,

$$
I(\omega) \propto \operatorname{Re} \int_{0}^{\infty} \exp \left\{-\frac{\Delta^{2}}{\gamma^{2}}\left(e^{-\gamma t}-1+\gamma t\right)-i \omega t\right\} d t
$$

where $\Delta$ is the amplitude (variance) of the modulation frequency of the charge-sensitive mode. In this case the correlation time is equal to the inverse of the decay rate, $\tau=1 / \gamma$. When $\tau \Delta>>1$ and $\tau \Delta<<1$, the lineshape approaches a Gaussian and a Lorentzian, respectively. Figure 4 shows the spectral line 
shape of these two cases plotted as a function of $\omega \Delta$ for various $\tau \Delta$ with $\omega_{1 / 2}=0$. In both cases, the line shape is determined by the parameter $\tau \Delta$. When both $\tau$ and $\Delta$ vary with temperature, it is difficult to determine them from an analysis of the line shape.

\section{3. $\beta^{\prime \prime}-(B E D T-T T F)(T C N Q)$}

\subsection{Crystal Structure and Superlattice}

$\beta^{\prime \prime}$-(BEDT-TTF)(TCNQ) has a segregated stack structure, in which BEDT-TTF and TCNQ are regularly stacked along the $c$ axis. The degree of the charge transfer of (BEDT-TTF) ${ }^{+\rho}(\mathrm{TCNQ})^{-\rho}$ is estimated to be $\rho=0.5$ from the frequency shift of $v_{21}, v_{34}$, and $v_{4}$ of TCNQ [61]. Therefore, this compound is regarded as a quarter-filled system. BEDT-TTF has the largest transfer integral along the side-by-side direction ( $a$ axis) and TCNQ has the largest transfer integral along the stacking direction [62]. The hopping interaction between BEDT-TTF and TCNQ along the $b$ axis is negligibly small. These theoretical predictions are qualitatively supported by the anisotropic optical conductivity [61]. Very weak superlattice spots are reported in ref. [61]. The observation of vibronic bands of TCNQ only in the E $\| c$ optical conductivity suggests that TCNQ is dimerized whereas BEDT-TTF is not. Based on these spectroscopic results and assuming the space group $P \overline{1}$, the proposed model of this superstructure is shown in Figure 5. TCNQ is connected by a center of symmetry ( $4 k_{\mathrm{F}}$ modulation) and BEDT-TTF is located on the center of symmetry. Therefore, the two TCNQs in the unit cell are crystallographically equivalent, whereas the two BEDT-TTFs are non-equivalent. These superlattice spots disappear at around $170 \mathrm{~K}$. However, the vibronic bands of TCNQ still remain after the superlattice spots vanish. This result implies that the short-range ordered $4 k_{\mathrm{F}}$ modulation remains in the TCNQ stack or another superlattice forms. According to the X-ray diffraction study by Nogami, a new superlattice spot for $2 a \times 2 c$ was found below $170 \mathrm{~K}$. [63]. In any case, the valence electron in the TCNQ stack is localized within the dimer, and the valence electron in the pseudo-one-dimensional BEDT-TTF band is responsible for the charge carriers.

$\beta^{\prime \prime}$-(BEDT-TTF)(TCNQ) is metal-like down to $1.8 \mathrm{~K}$ with three anomalies at $\sim 170 \mathrm{~K}, \sim 80 \mathrm{~K}$, and $\sim 20 \mathrm{~K}[62,64]$. The magneto-resistance below $20 \mathrm{~K}$ was extensively studied by Yasuzuka et al. They found five distinct small Fermi surfaces through the Shubnikov-de-Haas oscillations [65-67]. A quasi-one-dimensional periodic orbit resonance was found through the magneto-optical measurement $[68,69]$. The magneto-resistance and magneto-optical experiments demonstrate the existence of the Fermi surface below $20 \mathrm{~K}$, which implies that the ground state is a Fermi liquid metal. On the other hand, charge disproportionation with an amplitude of $\Delta \rho \sim 0.6$ was reported at room temperature based on the observation of the splitting of charge-sensitive $v_{27}$ mode of BEDT-TTF [70]. Such a large amplitude at high-temperature indicates a localized nature, so suggesting a phase change between the high- and low-temperature regions. 
Figure 5. Arrangement of molecules in $\beta^{\prime \prime}$-(BEDT-TTF)(TCNQ). Average lattice (dotted line; crystal axes are $\boldsymbol{a}, \boldsymbol{b}$, and $\boldsymbol{c}$ ), superlattice above $170 \mathrm{~K}$ (solid line; crystal axes are $\boldsymbol{a}^{\prime}$, $\boldsymbol{b}^{\prime}$, and $\boldsymbol{c}^{\prime}$ ), and superlattice $2 a \times 2 c$ below $170 \mathrm{~K}$ (dash dot line; crystal axes are $\boldsymbol{a}^{\prime \prime}, \boldsymbol{b}^{\prime \prime}$, and $\boldsymbol{c}^{\prime \prime}$ ). Arrangement in (a) BEDT-TTF (ET) and TCNQ; (b) BEDT-TTF; and (c) TCNQ.

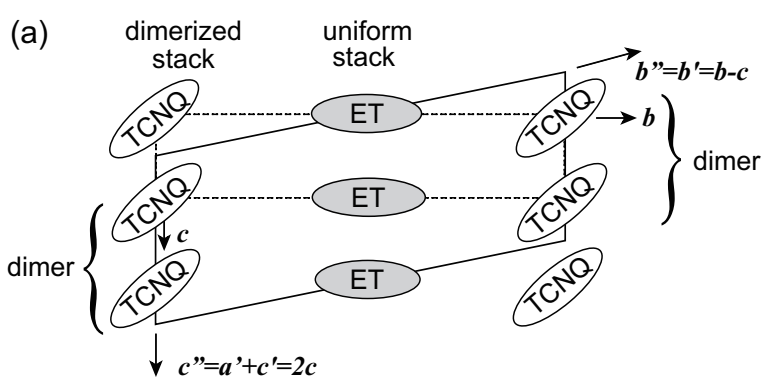

(b)

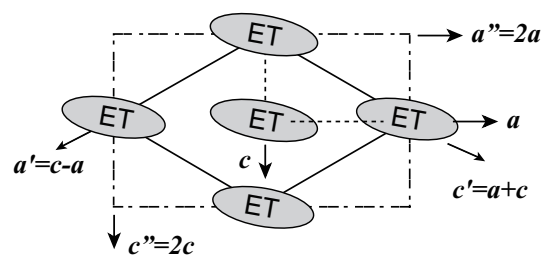

(c)

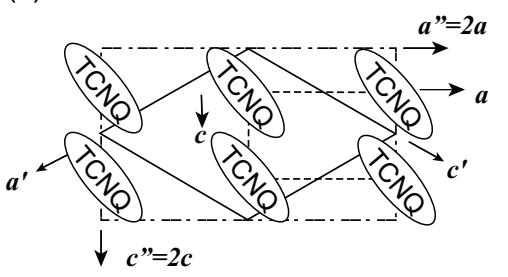

\subsection{Crossover from Uniform Metal to Charge-Ordered Metal}

Uruichi et al., have discussed the infrared, Raman, and optical conductivity of $\beta$ "-(BEDT-TTF)(TCNQ) [61]. Figure 6 shows the infrared-active $v_{27}$ mode and Raman active $v_{2}$ mode of BEDT-TTF along with the electrical resistivity. Both charge-sensitive modes, $v_{27}$ and $v_{2}$, appear as a single band at $\rho \sim 0.5$ below $100 \mathrm{~K}$. Upon increasing the temperature, they split into high-frequency and low-frequency modes. This splitting is comparable with $v_{27}$ and $v_{2}$ in the CO state of $\theta$-(BEDT-TTF) $)_{2}$ RbZn $[71,72]$. This splitting clearly shows the charge disproportionation from uniform site charge with $\Delta \rho \sim 0$ to two different site charges with an amplitude of $\Delta \rho \sim 0.6$. Above $150 \mathrm{~K}$, these two sites appear to correspond to the two non-equivalent sites in the superlattice (center and corner sites in Figure 5b). However, the intensity of the superlattice reflection is extremely weak compared with Bragg reflections. This observation means that the two independent sites are approximately equivalent. Therefore, this charge disproportionation seems not to be long-range ordered, and probably the short-range ordered site charges are dynamically fluctuating. The correlation time was estimated to be $\tau>\sim 0.3$ ps at room temperature assuming a two-states-jump model [61]. This will be further discussed below along with the optical conductivity. On the other hand, the charge-sensitive modes $v_{21}, v_{34}$, and $v_{4}$ of TCNQ show no change. This observation is consistent with the speculation that the valence electrons of TCNQ are localized within the TCNQ dimer over the whole temperature range.

Figure 7 shows the optical conductivity polarized along the $a$ axis, which is the direction of the maximum transfer integral between BEDT-TTF. The temperature dependence is peculiar in that the spectral weight shifts to the low-frequency region on decreasing temperature. At the same time the resistivity also decreases. This characteristic spectral change seems to be interpreted by the theoretical model of Merino et al. [40]. They computed the optical conductivity for a quarter-filled square lattice with various $V / t$ values under the condition of $U / t=20$, where $U, V$, ant $t$ are the on-site Coulomb 
energy, intersite Coulomb energy, and intersite hopping energy, respectively. When $V / t$ exceeds the critical value $(V / t)_{\mathrm{c}}$, a $\mathrm{CO}$ state is more stable than a metallic state. According to their theory, the density of states in a metallic phase $\left(V / t<(V / t)_{\mathrm{c}}\right)$ is triply peaked at around the Fermi energy (See the inset of Figure 7). From a low energy, these peaks correspond to the incoherent band associated with the lower Hubbard band, quasiparticle band, and upper incoherent band. When $V / t$ increases, the quasiparticle peak in the density of states decreases, and finally vanishes in the CO state $\left(V / t>(V / t)_{c}\right)$. Consequently the optical conductivity consists of three terms, $\sigma(\omega)=\sigma_{\mathrm{D}}(\omega)+\sigma_{\mathrm{IQ}}(\omega)+\sigma_{\mathrm{II}}(\omega)$, where $\sigma_{\mathrm{D}}(\omega)$ is the Drude term that peaks at $\omega=0$ which corresponds to the excitation within the quasiparticle band, $\sigma_{\mathrm{IQ}}(\omega)$ is the optical transition between the incoherent bands and the quasiparticle band, and $\sigma_{\text {II }}(\omega)$ is the optical transition between the incoherent bands (transition between the neighbor sites in real space description) [73]. In the CO state, the gap is open, so that $\sigma_{\mathrm{D}}(\omega)$ and $\sigma_{\mathrm{IQ}}(\omega)$ vanish. When $V / t$ decreases, the CO state is melted, and $\sigma_{\mathrm{D}}(\omega)$ and $\sigma_{\mathrm{IQ}}(\omega)$ gain the intensity and $\sigma_{\mathrm{II}}(\omega)$ loses the intensity. At the same time, the peaks of $\sigma_{\mathrm{IQ}}(\omega)$ and $\sigma_{\mathrm{II}}(\omega)$ shift toward lower energy. The observation of $\sigma_{\mathrm{D}}(\omega)$ is a sign of the coherent quasiparticle band and the intensity decrease and the low-energy shift of $\sigma_{\mathrm{IQ}}(\omega)$ and $\sigma_{\mathrm{II}}(\omega)$ corresponds to growth of the coherent band of the quasiparticle. This trend of $\sigma_{\mathrm{IQ}}(\omega)$ and $\sigma_{\mathrm{II}}(\omega)$ is seen in Figure $8 \mathrm{~b}$ with decreasing temperature. The Drude term $\sigma_{\mathrm{D}}(\omega)$ appearing below $600 \mathrm{~cm}^{-1}$ has not been measured in this compound. Probably $\sigma_{\mathrm{D}}(\omega)$ will quickly grows below $20 \mathrm{~K}$, as the dc conductivity increases remarkably below $20 \mathrm{~K}$ as shown in Figure 7.

Figure 6. Temperature dependences of the (a) $v_{27}$ mode (blue); (b) $v_{2}$ (blue) and $v_{3}$ (green) modes; and (c) electrical resistivity of BEDT-TTF in $\beta^{\prime \prime}-($ BEDT-TTF)(TCNQ). Note that the charge-sensitive modes merge into a single band on decreasing the temperature. This figure is modified from Figures 3,4,7 in ref. [61].
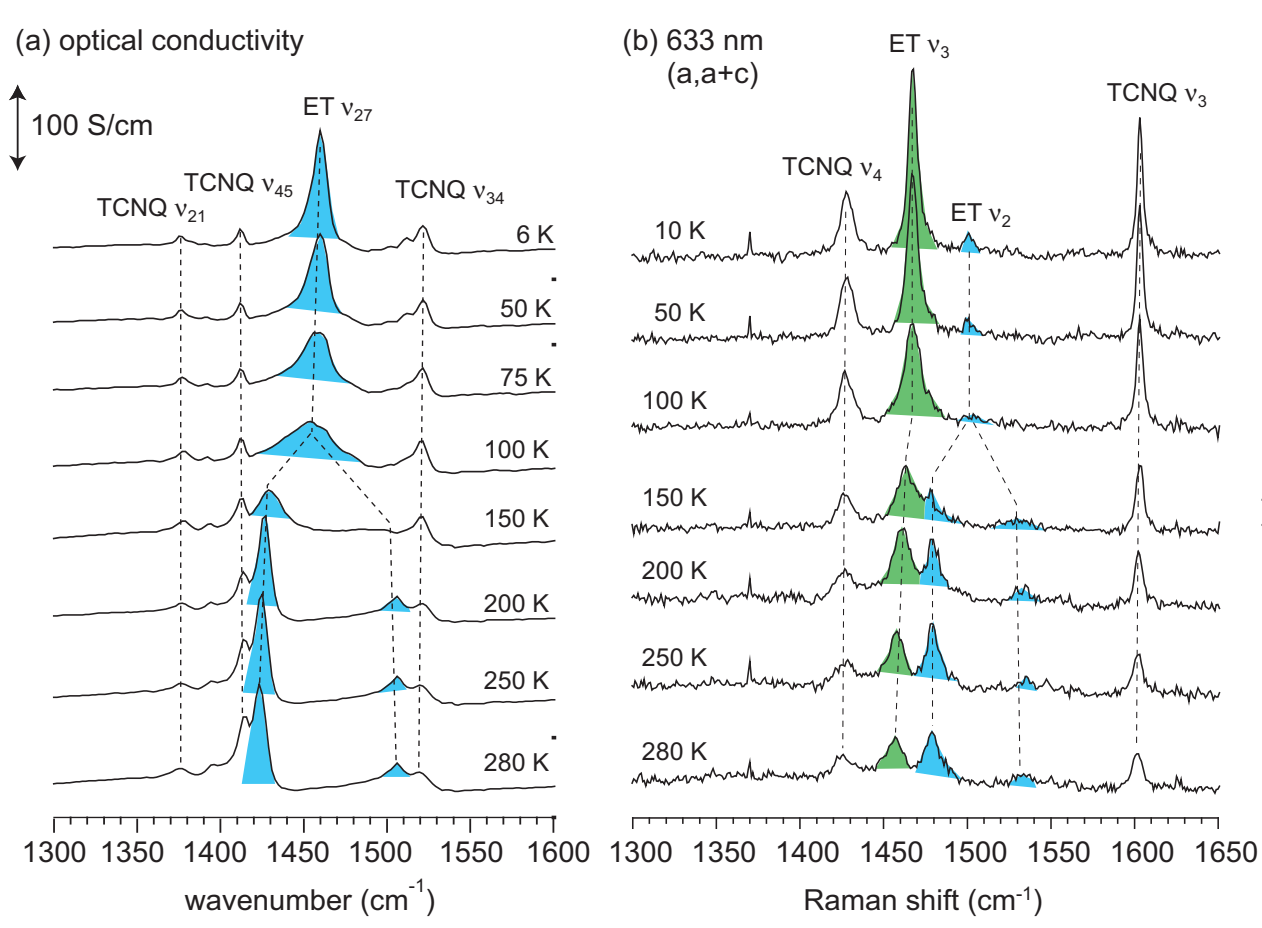

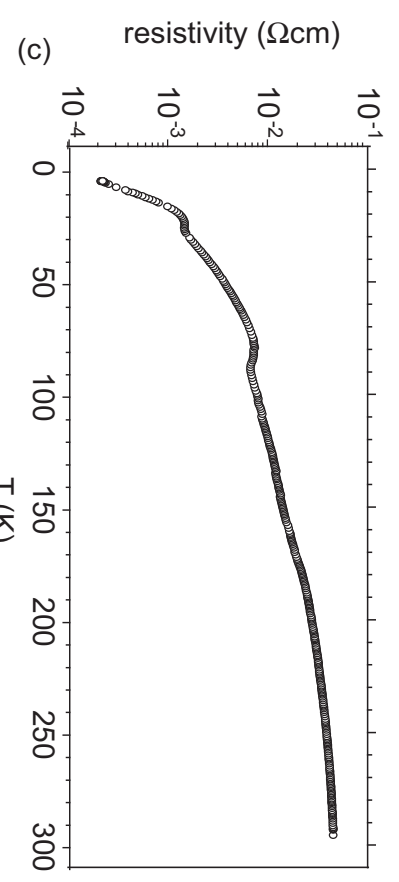


Figure 7. Temperature dependence of the E\|a optical conductivity of $\beta$ "-(BEDT-TTF) (TCNQ). Circles at zero wavenumber denote the dc conductivity at several temperatures. The inset shows the schematic figure of density of states in the quarter-filled band system. See the text for the meaning of the electronic transitions, $\sigma_{\mathrm{D}}(\omega), \sigma_{\mathrm{IQ}}(\omega)$, and $\sigma_{\mathrm{II}}(\omega)$.

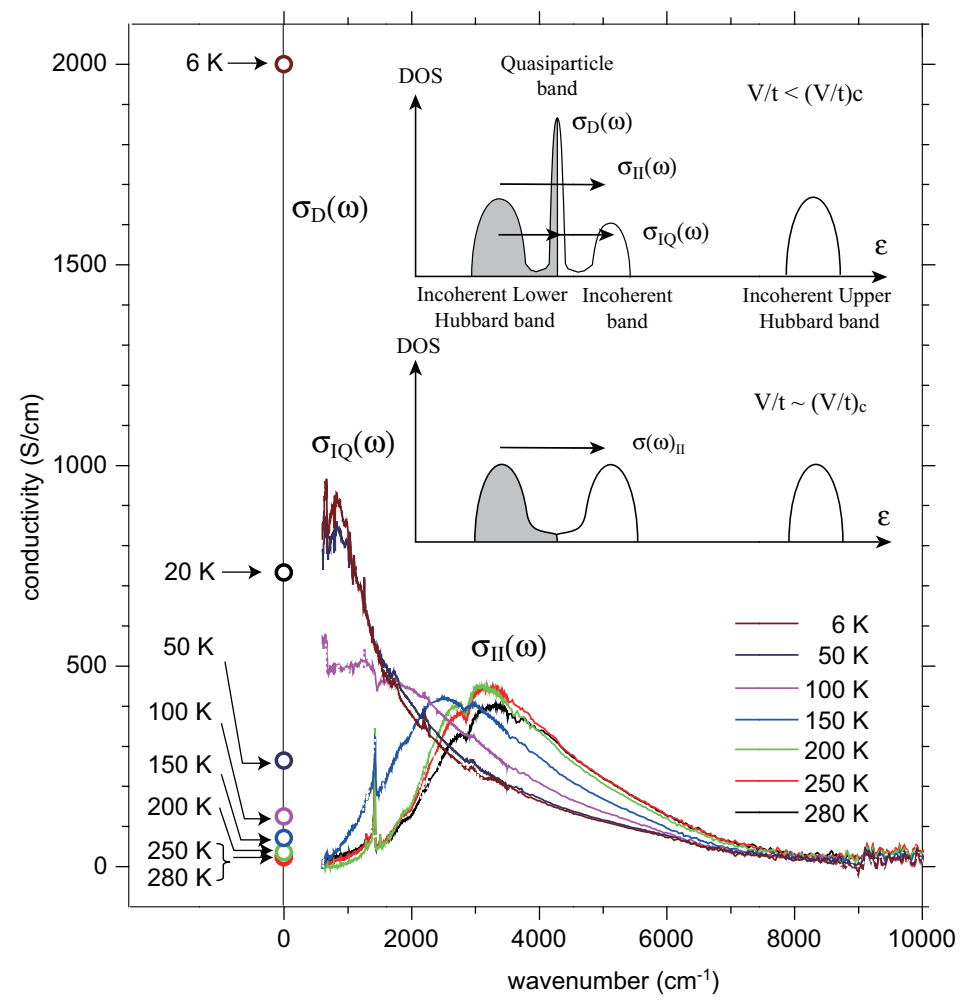

Figure 8. Temperature dependences of the following quantities of $\beta^{\prime \prime}$-(BEDT-TTF)(TCNQ). (a) electrical resistivity; (b) peak frequency of electronic transition; (c) partial effective hole number; (d) peak frequency of $v_{27}$, where squares and triangles are the data of independent experiment; and (e) product of correlation time and splitting width of $v_{27}$. Lines are included as guide to the eye. The inset of Figure 8 a shows that the resistivity below $20 \mathrm{~K}$ follows the relation for a Fermi liquid, $\rho=\rho_{0}+\mathrm{AT}_{2}$. Figure 8a,b are taken from Figure 7 of ref. [61].

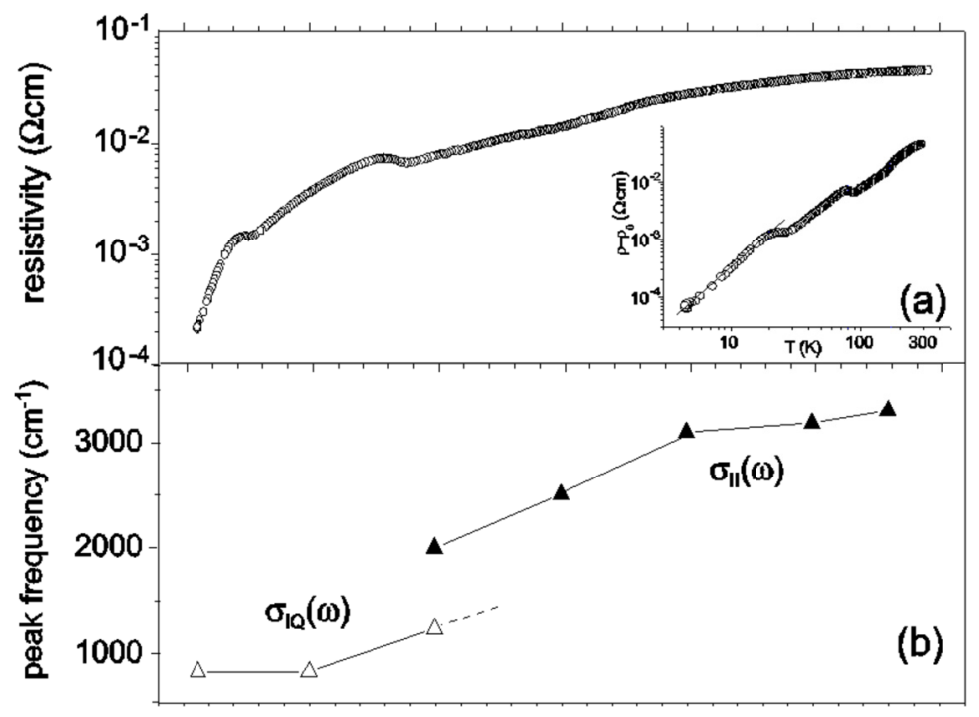


Figure 8. Cont.

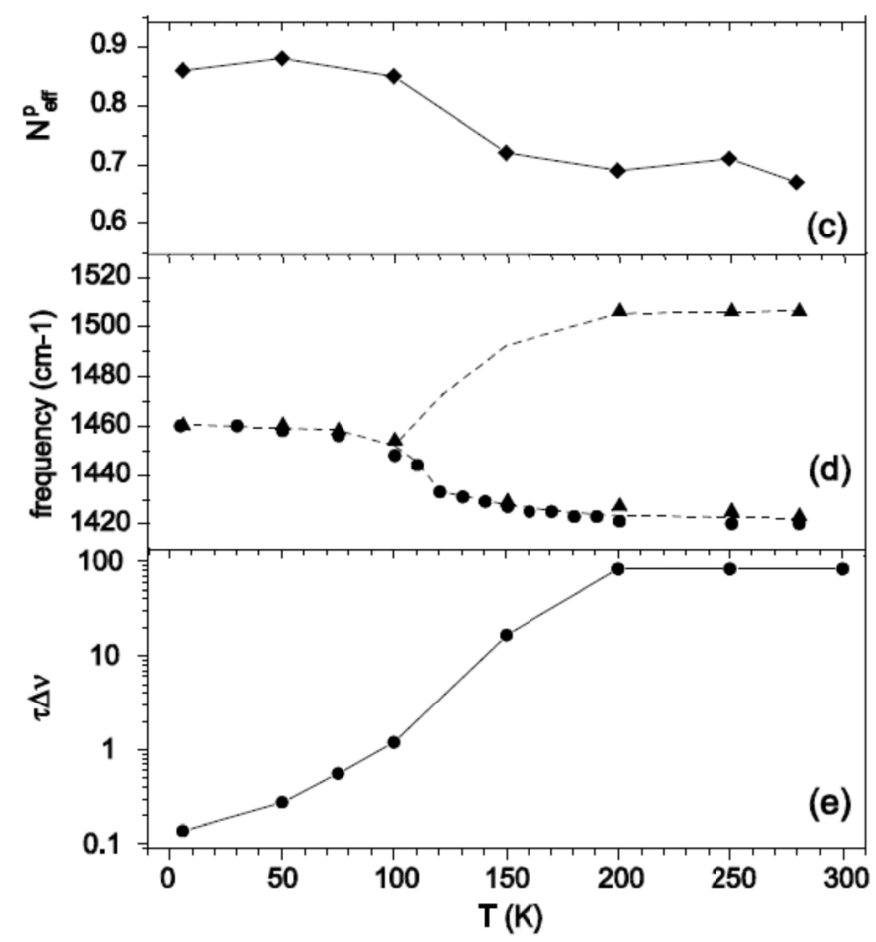

According to the charge correlation function $C(\boldsymbol{q}, \omega)$ near $\mathrm{CO}$ calculated by Merino et al., the excitation energy $\omega$ approaches zero and the correlation length diverges [40,41], when the parameters $V / t$ approach the critical value for long-range CO. Accordingly, the amplitudle of CO fluctuation $\Delta \rho$ increases and the correlation time $\tau$ is expected to diverge. This trend is seen in Figure 8e on increasing the temperature, where the product $\tau \Delta v$ is estimated from the two-states-jump model as explained in Section 2.4. Therefore, together with the optical conductivity, $\beta{ }^{\prime \prime}$-(BEDT-TTF)(TCNQ) approaches the long-range $\mathrm{CO}$ state with increasing temperature. Above $200 \mathrm{~K}$ the increase in the product $\tau \Delta v$ levels off, and the optical conductivity appears to have a gap. If this compound attains a long range order above $200 \mathrm{~K}$, the charge-rich and charge-poor molecules are fixed at crystallographically independent sites. In spite of the large amplitude of $\mathrm{CO}$, the two independent sites are approximately equivalent, as described in the first paragraph of this section. This result suggests that short-range charge order fluctuates, and it is not long-range ordered above $200 \mathrm{~K}$. The absence of long-range order as well as the sizable dc conductivity with $\mathrm{d} \rho / \mathrm{dT}>0$ in this temperature range, as shown in Figure 8a, suggests the existence of a finite density of states at the Fermi level as schematically drawn in the inset in Figure 7. The apparent optical gap is regarded as a pseudogap. Therefore the high-temperature state is considered to be a charge-ordered metal [74]. The amplitude $\Delta \rho \sim 0.6$ and correlation time $\tau>\sim 0.3$ ps may characterize the charge-ordered metal of this compound. The optical conductivity below $600 \mathrm{~cm}^{-1}$ is necessary to clarify the finite density of state near the Fermi level.

In contrast, at low temperatures, the $\mathrm{CO}$ fluctuation is significantly suppressed, as $\tau \Delta v$ is decreased by three orders of magnitude. Merino predicted a transition between a uniform metal and a charge-ordered metal [74]. As shown in Figure 8d, the spectral variation of $v_{27}$ looks to be continuous at around $100 \mathrm{~K}$. Therefore, this compound shows a crossover from a charge-ordered metal to a uniform metal upon 
lowering the temperature. Comparing with the theoretical model, it turns out that decreasing temperature corresponds to a decrease of $V / t$. Although the lattice contraction increases both $V$ and $t$, the latter is more sensitive to an intermolecular distance. Therefore, lattice contraction is responsible for this crossover behavior. Actually, the increase in $t$ is indicated by an increase in the partial effective number, which is defined by the following equation,

$$
N_{e f f}^{p}=\frac{2 m}{\pi e^{2} N} \int_{\omega_{c 1}}^{\omega_{c 2}} \sigma(\omega) d \omega
$$

where $m$ and $e$ are the electron mass and electron charge respectively, $N$ is the hole density of BEDT-TTF, $\omega_{\mathrm{c} 1}=600 \mathrm{~cm}^{-1}$ and $\omega_{\mathrm{c} 2}=9000 \mathrm{~cm}^{-1}$ are the cut-off frequencies, and $\sigma(\omega)$ are the cut-off frequencies, $N^{\mathrm{p}}$ eff increases by about $30 \%$ from $280 \mathrm{~K}$ to $6 \mathrm{~K}$ as shown in Figure $8 \mathrm{c}$, though the spectral weight below $600 \mathrm{~cm}^{-1}$ is not taken into account. The role of lattice contraction on the electronic state is also supported by high pressure experiment. When a hydrostatic pressure is applied to this compound at room temperature, the Raman spectrum shows a similar variation as a decrease in the temperature. The split $v_{2}$ modes merge at about 2 kbar [61], that is, the charge-ordered metal changes into a uniform metal due to lattice contraction. Possibly, a quasiparticle band is formed below $20 \mathrm{~K}$ because of the high conductivity and Fermi liquid behavior of transport properties [65-69]. To confirm this, the Drude response should be examined in the optical conductivity below $600 \mathrm{~cm}^{-1}$. Finally, the structural change in the superlattice at $\sim 170 \mathrm{~K}$ and anomalies in resistivity at $\sim 80 \mathrm{~K}$ and $\sim 20 \mathrm{~K}$ seem to have no influence on the optical conductivity and the line shape of the charge-sensitive mode. More extensive investigation is necessary for the understanding of this compound.

\section{4. $\theta-(B E D T-T T F){ }_{2} X$}

The $\theta$-type BEDT-TTF salts, $\theta$-(BEDT-TTF) ${ }_{2} \mathrm{X}$, have a herringbone arrangement of BEDT-TTF, forming a two-dimensional quarter-filled system. This family includes metallic compounds and several compounds which undergo a charge-ordering (CO) phase transition. The electronic phase diagram of this family has been composed employing the dihedral angle $\varphi$ between the adjacent BEDT-TTF molecules (See Figure 9), which is related to the bandwidth [74]. The dihedral angle was subsequently linked to $t_{c} / t_{p}$ to explain the phase diagram, where $t_{c}$ and $t_{p}$ are the transfer integrals along the $c$ (stacking) direction and $\boldsymbol{a} \pm \boldsymbol{c}$ (diagonal) directions forming a triangular lattice [75-78]. Watanabe discussed this phase diagram using $t_{c} / t_{p}$ as follows [78]. As the dihedral angle $\varphi$ decreases, the bandwidth increases [79], and the ground state is stabilized in the order of a stripe $\mathrm{CO}$, frustrating $\mathrm{CO}$ between the stripe and the threefold lattice, and uniform metal. Accordingly, the temperature range of the highly conductive phase expands to the low-temperature region, as $\varphi$ is decreased. In the small $\varphi$ region, $\mathrm{X}=\mathrm{I}_{3}\left(\varphi=100^{\circ}\right)[80]$ and $\mathrm{X}=\mathrm{CsZn}(\mathrm{SCN})_{4}\left(\varphi=104^{\circ}\right)$ [81] salts, the high-temperature phase is metallic, with $\mathrm{d} \rho / \mathrm{dT}>0$, whereas it is non-metallic, with $\mathrm{d} \rho / \mathrm{dT}<0$ above the $\mathrm{CO}$ transition temperature in the large $\varphi$ region. The activation energy increases with increasing $\varphi$ as follows: $0.02 \mathrm{eV}$ for $\mathrm{X}=\mathrm{RbZn}(\mathrm{SCN})_{4}\left(\varphi=111^{\circ}\right)$ [81], $0.08 \mathrm{eV}$ for orthorhombic $\operatorname{TlZn}(\mathrm{SCN})_{4}\left(\varphi=117^{\circ}\right)[81]$, $0.11 \mathrm{eV}$ for monoclinic $\operatorname{TlZn}(\mathrm{SCN})_{4}\left(\varphi=121^{\circ}\right)$ [81], and $0.17-0.19 \mathrm{eV}$ for $\mathrm{Cu}_{2}(\mathrm{CN})\left[\mathrm{N}(\mathrm{CN})_{2}\right]_{2}$ $\left(\varphi=132^{\circ}\right)[82]$. Hereafter, $\theta-(\mathrm{BEDT}-\mathrm{TTF})_{2} \mathrm{MM}^{\prime}(\mathrm{SCN})_{4}$ and $\theta-(\mathrm{BEDT}-\mathrm{TTF})_{2} \mathrm{X}$ are abbreviated as $\theta-\mathrm{MM}^{\prime}$ and $\theta-\mathrm{X}$, respectively. 
Figure 9. Phase diagram of $\theta-(\mathrm{BEDT}-\mathrm{TTF})_{2} \mathrm{X}$ [79]. The squares and the circle represent the charge-ordering phase transition and the superconducting phase transition, respectively.

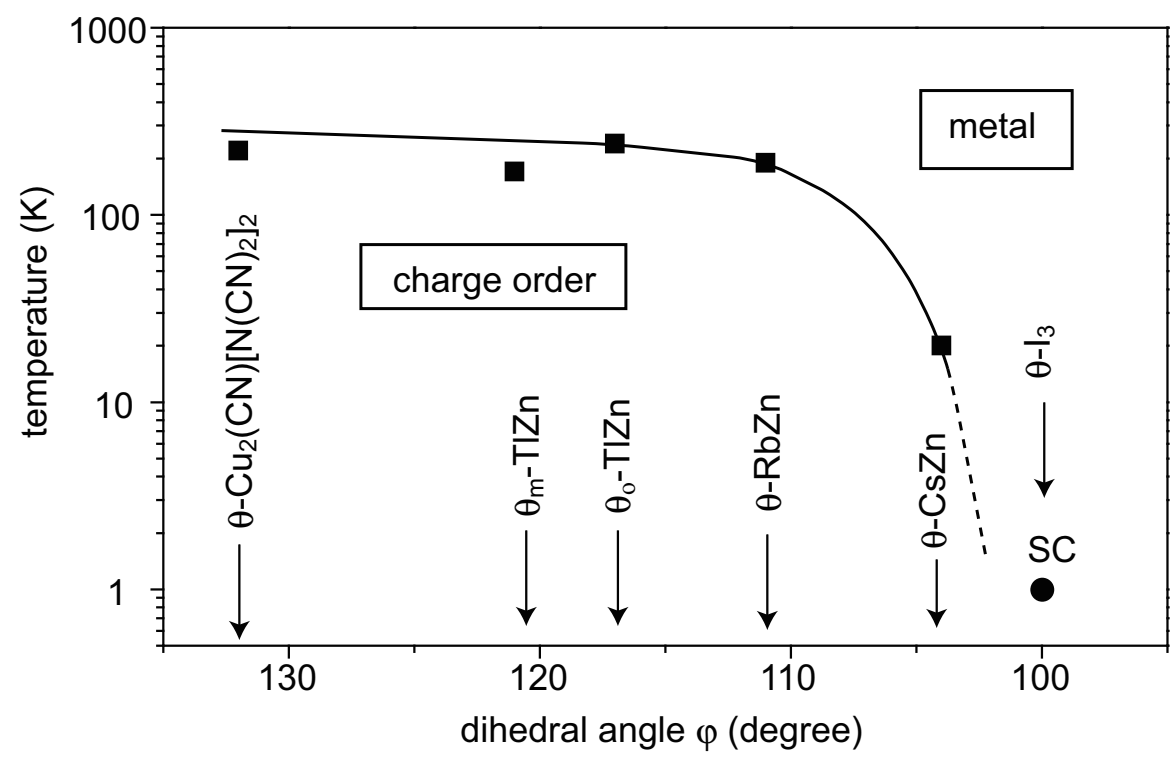

dihedral angle

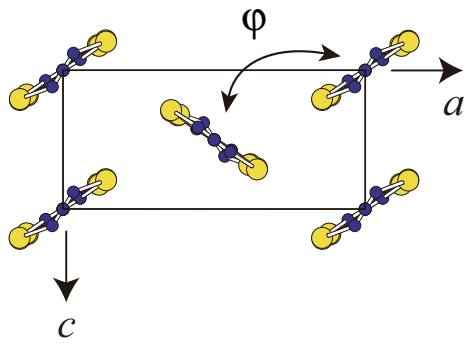

\subsection{Charge-Ordered Phase of $X=R b Z n(S C N)_{4}, \operatorname{TlZn}(S C N)_{4}, C u_{2}(C N)\left[N(C N)_{2}\right]_{2}$}

$\theta$-RbZn undergoes the $\mathrm{CO}$ phase transition accompanying a structural change, and this compound has been very extensively studied. As shown in the left panel of Figure 10, Raman-active $v_{2}$ and $v_{3}$ modes show remarkable change in the $\mathrm{CO}$ transition. Yamamoto et al., analyzed the spectra comparing them with the spectra for the ${ }^{13} \mathrm{C}$-substituted compound, in which the carbon atoms in the central bridge $\mathrm{C}=\mathrm{C}$ bond are substituted by ${ }^{13} \mathrm{C}[72]$. Since the $v_{2}$ mode is mainly the $\mathrm{C}=\mathrm{C}$ stretching vibration in the five-member rings and the $v_{3}$ mode is mainly the $\mathrm{C}=\mathrm{C}$ stretching vibration of the central bridge $\mathrm{C}=\mathrm{C}$ bond, (See Figure 1) the isotope shift of the latter mode is expected to be significantly larger than the former. Utilizing this property, they assigned all the observed bands as shown in Figure 10. Actually, the isotope shifts of $v_{3 \mathrm{~A}}, v_{3 \mathrm{~B}}, v_{3 \mathrm{~B}}$, and $v_{3 \mathrm{~A}}$, are much larger than those of $v_{2 \mathrm{P}}$ and $v_{2 \mathrm{R}}$, while $v_{4}$, and $v_{5}$ show no isotope shift [72]. It becomes clear from this assignment that the $v_{2}$ mode splits into two and the $v_{3}$ mode split into four [83]. As we explained in Section 2.3, the $v_{2}$ mode splits owing to the charge difference and the $v_{3}$ mode splits owing to the EMV mechanism. From the splitting of $v_{2}$, the site-charge difference or amplitude of $\mathrm{CO}$ is estimated to be $\Delta \rho \sim 0.6$. This large amplitude is approximately consistent with the site-charge ratio $\rho_{\mathrm{P}}: \rho_{\mathrm{R}}=1: 6$ estimated from ${ }^{13} \mathrm{C}$-NMR experiment $[15]$. From the splitting of $v_{3}$, it is concluded that the single conducting layer in the unit cell contains four molecules. This means that the unit cell is doubled below the CO phase transition.

As shown in the right panel of Figure 10, the four $v_{3}$ modes are classified into two groups by the polarization of the incident and scattered light. The two $v_{3 \mathrm{~A}}$ modes and two $v_{3 \mathrm{~B}}$ modes, respectively, have the character of species $\mathrm{A}$ and $\mathrm{B}$ in the $C_{2}$ symmetry. This means that the four molecules in a conducting layer are classified into two groups, and in each group, two equivalent molecules are connected by a screw axis. These groups correspond to charge-rich and charge-poor molecules. Therefore, the charge-rich (charge-poor) molecules are arranged to form a horizontal stripe. This conclusion is consistent with the space group $P 2{ }_{1} 2_{1} 2_{1}$ which was determined by the low-temperature X-ray diffraction measurements [84]. The factor group of $P 2_{1} 2_{1} 2_{1}$ is $D_{2}$, which is reduced to $C_{2}$ for a 
single conducting layer [85]. The horizontal stripe in the $\theta-\mathrm{RbZn}$ salt is claimed by Tajima et al., through the analysis of the mid-infrared band in the optical conductivity [86]. Based on the mode of the horizontal stripe, Suzuki et al. [85] and Yamamoto et al. [57] conducted a numerical calculation of the splitting of $v_{2}$ and $v_{3}$. According to their analysis, the four vibronic modes are expressed by the following symmetry coordinates,

$$
\begin{aligned}
& Q_{A 1}=\left\{a\left(Q_{P 1}+Q_{P 2}\right)+\sqrt{1-a^{2}}\left(Q_{R 1}+Q_{R 2}\right)\right\} / \sqrt{2} \\
& Q_{A 2}=\left\{\sqrt{1-a^{2}}\left(Q_{P 1}+Q_{P 2}\right)-a\left(Q_{R 1}+Q_{R 2}\right)\right\} / \sqrt{2} \\
& Q_{B 1}=\left\{b\left(Q_{P 1}-Q_{P 2}\right)+\sqrt{1-b^{2}}\left(Q_{R 1}-Q_{R 2}\right)\right\} / \sqrt{2} \\
& Q_{B 2}=\left\{\sqrt{1-b^{2}}\left(Q_{P 1}-Q_{P 2}\right)-b\left(Q_{R 1}-Q_{R 2}\right)\right\} / \sqrt{2}
\end{aligned}
$$

where $Q_{\mathrm{P} 1}$ and $Q_{\mathrm{P} 2}\left(Q_{\mathrm{R} 1}\right.$ and $\left.Q_{\mathrm{R} 2}\right)$ are the normal coordinates of charge-poor (charge-rich) site. The different character between $v_{2}$ and $v_{3}$ is ascribed to the difference in EMV coupling constants, which are small for $v_{2}$ and large for $v_{3}$ compared to transfer integrals. When the normal coordinate has $a_{\mathrm{g}}$ symmetry in a free molecule, $Q_{\mathrm{A} 1}$ and $Q_{\mathrm{A} 2}$ have A symmetry and $Q_{\mathrm{B} 1}$ and $Q_{\mathrm{B} 2}$ have B symmetry. In the case of $v_{2}$ mode, $a \approx 1$ and $b \approx 1$, since the coupling constant is small, in other words, the interaction between charge-rich and charge-poor site is small. Therefore, both $v_{2 \mathrm{P}}\left(v_{\mathrm{A} 1}, v_{\mathrm{B} 1}\right)$ and $v_{2 \mathrm{R}}\left(v_{\mathrm{A} 2}, v_{\mathrm{B} 2}\right)$ are doubly degenerate. On the other hand, the four $v_{3}$ modes, $v_{3 \mathrm{~A}}\left(v_{\mathrm{A} 1}, v_{\mathrm{A} 2}\right)$ and $v_{3 \mathrm{~B}}\left(v_{\mathrm{B} 1}, v_{\mathrm{B} 2}\right)$ are split through the strong EMV interaction.

Figure 10. (left panel) Temperature dependence of the Raman spectrum and the electrical resistivity of $\theta-(\mathrm{BEDT}-\mathrm{TTF})_{2} \mathrm{RbZn}(\mathrm{SCN})_{4}$. The $v_{2}$ and $v_{3}$ modes are shown by blue and green colors. The inset shows the herringbone arrangement of BEDT-TTF with respect to the crystallographic axes; (right panel) Polarized Raman spectrum of $\theta-\mathrm{RbZn}$ measured at $20 \mathrm{~K}$. The four $v_{3}$ modes (green) in the top panel are classified into two groups by polarization. The Raman spectra are modified from Figures 14,15 of ref. [24].
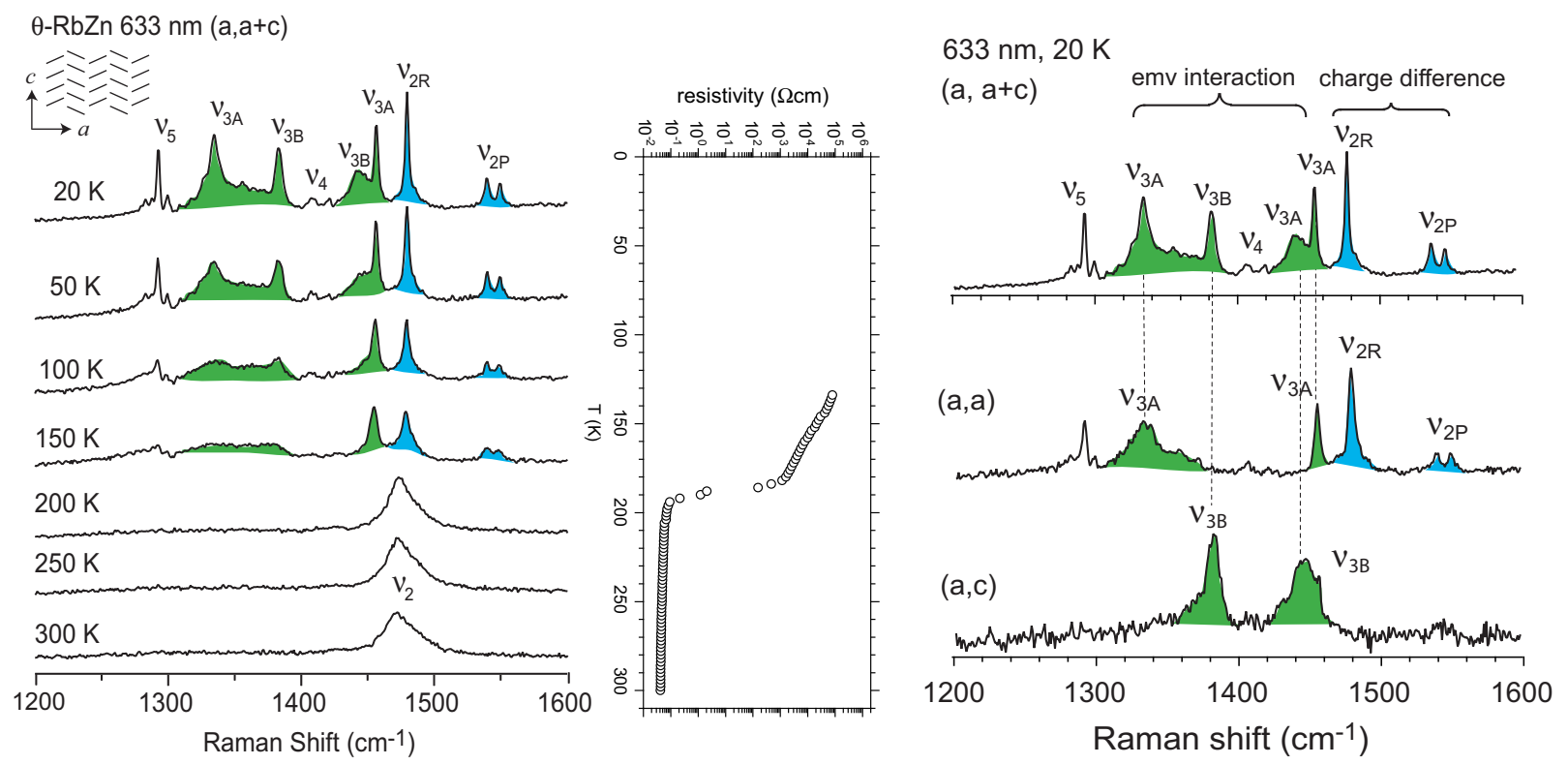
Similar analyses were conducted for $\theta_{\mathrm{o}}-\mathrm{TlZn}$ [85], $\theta_{\mathrm{m}}-\mathrm{TlZn}$ [85], and $\theta-\mathrm{Cu}_{2}(\mathrm{CN})\left[\mathrm{N}(\mathrm{CN})_{2}\right]_{2}$ [57] salts. Since $\theta_{0}-\mathrm{TlZn}$ and $\theta-\mathrm{Cu}_{2}(\mathrm{CN})\left[\mathrm{N}(\mathrm{CN})_{2}\right]_{2}$ are isostructural to $\theta-\mathrm{RbZn}$, the Raman spectra below $T_{\mathrm{CO}}$ are exactly the same as that of $\theta-\mathrm{RbZn}$ as shown in Figure 11 . However, $\theta_{\mathrm{m}}$-TlZn belongs to monoclinic system with space group $C 2$. Reflecting this structural difference, the Raman spectrum and electrical resistivity shown in Figure 12 are different from those of the orthorhombic salts [85]. The spectrum at $60 \mathrm{~K}$ is interpreted assuming pseudo inversion symmetry in the following way. The $v_{2}$ mode is split into the charge-rich and charge-poor sites, and two in-phase modes are observed among four $v_{3}$ modes. Based on this interpretation a diagonal stripe is claimed to be the CO pattern [85]. A diagonal stripe is theoretically predicted to be almost degenerate with a horizontal stripe. It is theoretically considered that the lattice distortion below $T_{\mathrm{CO}}$ stabilizes the horizontal stripe in $\theta-\mathrm{RbZn}$ [87]. If $\theta_{\mathrm{m}}$-TlZn takes a diagonal stripe, the low-temperature structure is expected to be distorted differently from $\theta-\mathrm{RbZn}$.

Figure 11. Temperature dependence of the Raman spectrum of $\theta_{0}-(\mathrm{BEDT}-\mathrm{TTF})_{2} \mathrm{TlZn}(\mathrm{SCN})_{4}$ along with the electrical resistivity. The inset shows the herringbone arrangement of BEDTTTF with respect to the crystallographic axes. The spectral change is almost exactly the same as that of $\theta-(\mathrm{BEDT}-\mathrm{TTF})_{2} \mathrm{RbZn}(\mathrm{SCN})_{4}$. The Raman spectrum is modified from Figure 4 of ref. [85].
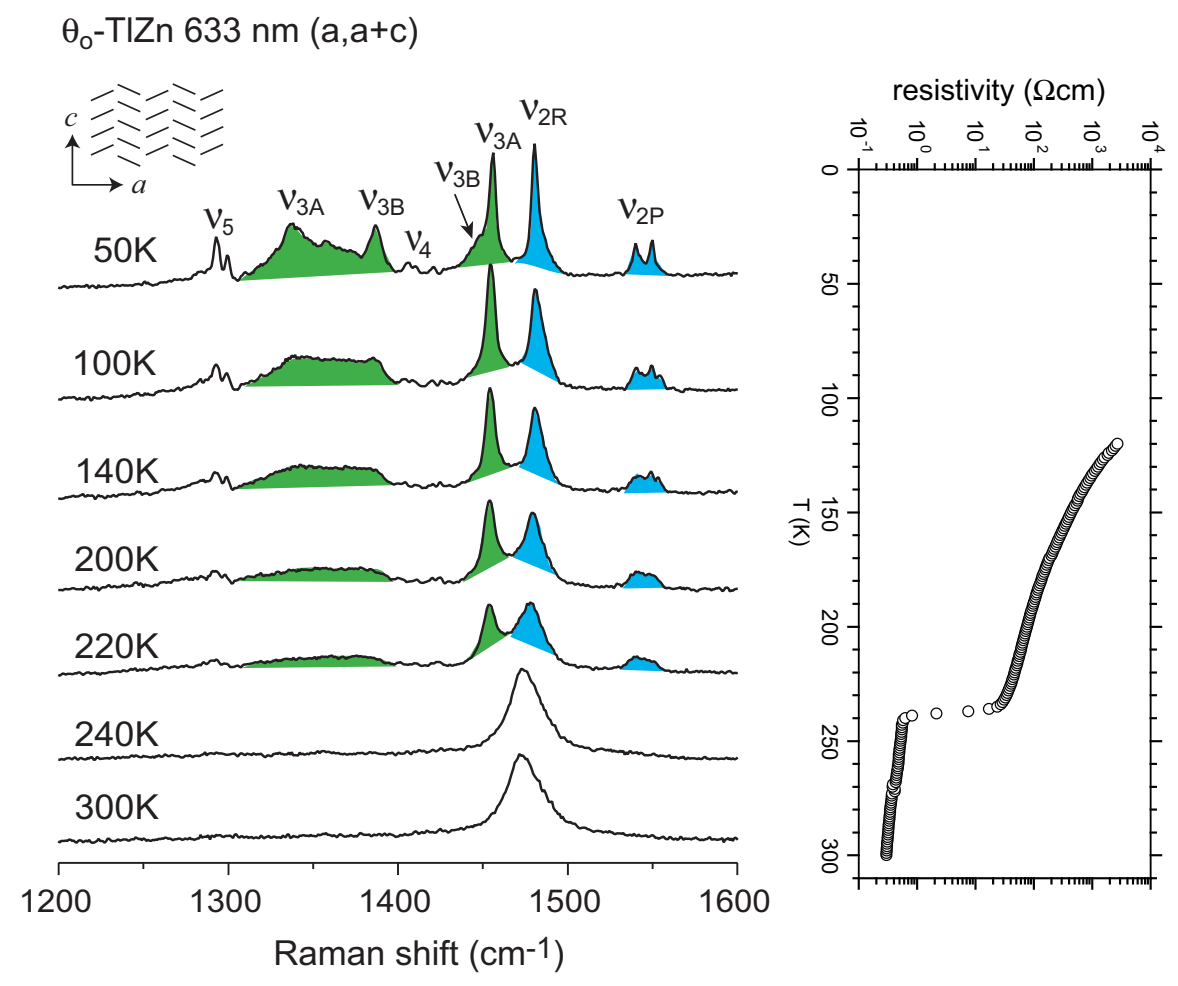
Figure 12. Temperature dependence of the Raman spectrum of $\theta$-(BEDT-TTF $)_{2}$ $\mathrm{Cu}(\mathrm{CN})\left[\mathrm{N}(\mathrm{CN})_{2}\right]_{2}$ (left) and $\theta_{\mathrm{m}}$-(BEDT-TTF $)_{2} \mathrm{TlZn}(\mathrm{SCN})_{4}$ (middle) along with the electrical resistivity. The inset shows the herringbone arrangement of BEDT-TTF with respect to the crystallographic axes. The spectral change and resistivity are rather continuous. The left spectrum is modified from Figure 5 of ref. [57]. The right spectrum is modified from Figure 7 of ref. [85].
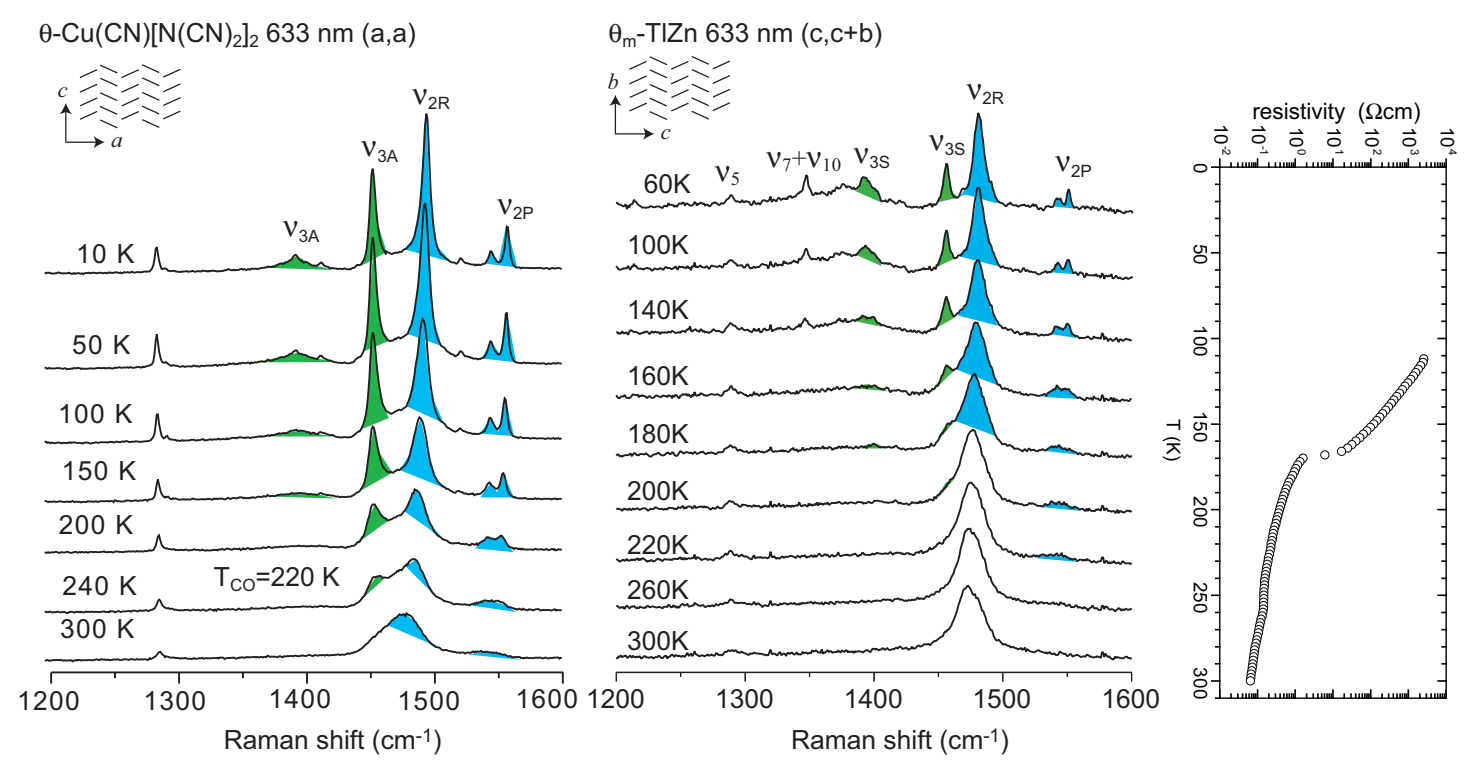

\subsection{Metallic Phase of $X=R b Z n(S C N)_{4}, T l Z n(S C N)_{4}, C u_{2} C N\left[N(C N)_{2}\right]_{2}$}

The high-temperature phase of $\theta-\mathrm{RbZ}$ is regarded as an unconventional metal. There is a finite density of states at the Fermi level, though the temperature derivative of resistivity is negative. This unusual behavior in the high-temperature phase of $\theta$ - RbZn was reported in ${ }^{13} \mathrm{C}$-NMR experiment. The linewidth shows a broadening as the temperature approaches $T_{\mathrm{CO}}$ [15]. Chiba analyzed this behavior, and reported the following results [88]. Above $T_{\mathrm{CO}}$, fluctuation of $\mathrm{CO}$ arises in space (the fractional charge is distributed from +0.3 to +0.7 ) and in time (extremely slow on the time scale of ${ }^{13} \mathrm{C}-\mathrm{NMR}$ ). This short-range ordered $\mathrm{CO}$ is related to the x-ray diffuse scattering at $\boldsymbol{q}_{1} \approx(1 / 4, k, 1 / 3)$, which corresponds to the short-range ordered $4 a \times 3 c$ superlattice (short-range ordered threefold CO) [84]. When the sample is rapidly cooled, the phase transition is suppressed [89]. This rapid-cooling state is regarded as a frozen state of the metal, and it is in a glassy state [90]. In this frozen state, another diffuse scattering at $\boldsymbol{q}_{2}=(0, k, 1 / 2)$ is found in addition to weak $\boldsymbol{q}_{1}$ [91]. The diffuse scattering at $\boldsymbol{q}_{2}$ corresponds to the short-range ordered $2 c$ superlattice (short-range ordered stripe $\mathrm{CO}$ ). As this metastable state relaxes, the volume fraction of the $2 c$ superlattice decreases, whereas the $4 a \times 3 c$ superlattice becomes dominant [92]. This result means that threefold $\mathrm{CO}$ and stripe $\mathrm{CO}$ are competing in the glassy state.

The Raman spectrum shows a single broad band above $T_{\mathrm{CO}}$ as shown in Figure 10 . This broad band is assigned to the $v_{2}$ mode based on the isotope shift of the ${ }^{13} \mathrm{C}$-substituted compound [93]. If the high-temperature phase is a metal, the $v_{2}$ mode should be observed between the $v_{2 \mathrm{P}}$ and $v_{2 \mathrm{R}}$ bands. However, the broad $v_{2}$ band above $T_{\mathrm{CO}}$ is located at the $v_{2 \mathrm{R}}$ band, and thus $v_{2 \mathrm{R}}$ is regarded as being hidden in the background. This result as well as the ${ }^{13} \mathrm{C}-\mathrm{NMR}$ data is direct evidence for the 
fluctuation of charge order in the metallic phase. The large splitting, $\Delta v_{2}=v_{2 \mathrm{P}}-v_{2 \mathrm{R}}$, means a large amplitude $(\Delta \rho \sim 0.6)$ of fluctuation. If the correlation time is very long on the time scale of vibrational spectroscopy as claimed by NMR [88], the line shape is regarded as the case of $\tau \Delta v_{2}>1$ (See Figure $4 a, \Delta$ $=\Delta v_{2} / 2$ ), that is, motional narrowing does not work. Therefore, Raman spectroscopy yields a snap shot of the slow fluctuation, and thus the broad linewidth reflects spatially inhomogeneous charge distribution. Accordingly, the line shape of the $v_{2}$ mode in the rapid-cooling state is almost exactly the same as that of the high-temperature spectrum [24,94]. The strong inhomogeneity probably comes from the short-range ordered $3 a \times 4 c$ superlattice, which involves 48 sites in the unit cell. On the other hand, in the case of ${ }^{13} \mathrm{C}-\mathrm{NMR}$, the line broadening due to the spatial inhomogenity is wiped out at higher temperature due to the motional narrowing, because the time scale of ${ }^{13} \mathrm{C}$-NMR is slower than that for vibrational spectroscopy. The line broadening comes back at low temperature due to the slower dynamics.

The optical conductivity of $\theta-\mathrm{RbZn}$ has been reported by Wang et al., down to $50 \mathrm{~cm}^{-1}$ [95]. As shown in Figure 13f,g, the optical conductivity above $200 \mathrm{~K}$ consists of a broad peak at around $2000 \mathrm{~cm}^{-1}$ with no Drude response. Theoretically, the $3 a \times 4 c$ superlattice is metallic with Fermi surfaces [76]. Nishimoto et al., calculated the optical conductivity of threefold lattice. They show that the Drude weight is significantly suppressed, and the spectral weight shifts to high-frequency excitation (See Figure 13a-e) [96]. The resultant small Drude response may be overdamped in the short-range ordered $3 a \times 4 c$ superlattice, which results in the pseudogap like optical conductivity. The optical conductivity in the metallic phase exhibits strong vibronic band of $v_{3}$ at $\sim 1300 \mathrm{~cm}^{-1}[72,95]$. As the vibronic band is forbidden in the structure of the metallic phase, this vibronic band is activated due to the local lattice distortion by the fluctuation of threefold and stripe $\mathrm{CO}$. In other words, the appearance of the vibronic band in $\theta$-type BEDT-TTF salts indicates the fluctuation of $\mathrm{CO}$ in the metallic phase.

Figure 13. Reflectivity and optical conductivity of $\theta-(\mathrm{BEDT}-\mathrm{TTF})_{2} \mathrm{RbZn}(\mathrm{SCN})_{4}$. and theoretical calculation of the optical conductivity of a triangular lattice. The arrows in (c), (d), and (e) denote the Drude peaks, which appear at finite energy because the open-end boundary condition is applied for a finite size cluster of $L_{a}=8$ and $L_{c}=6$ [96]. Figure 13a-e are taken from ref. [96], and Figure 13f,g are taken from ref. [95].
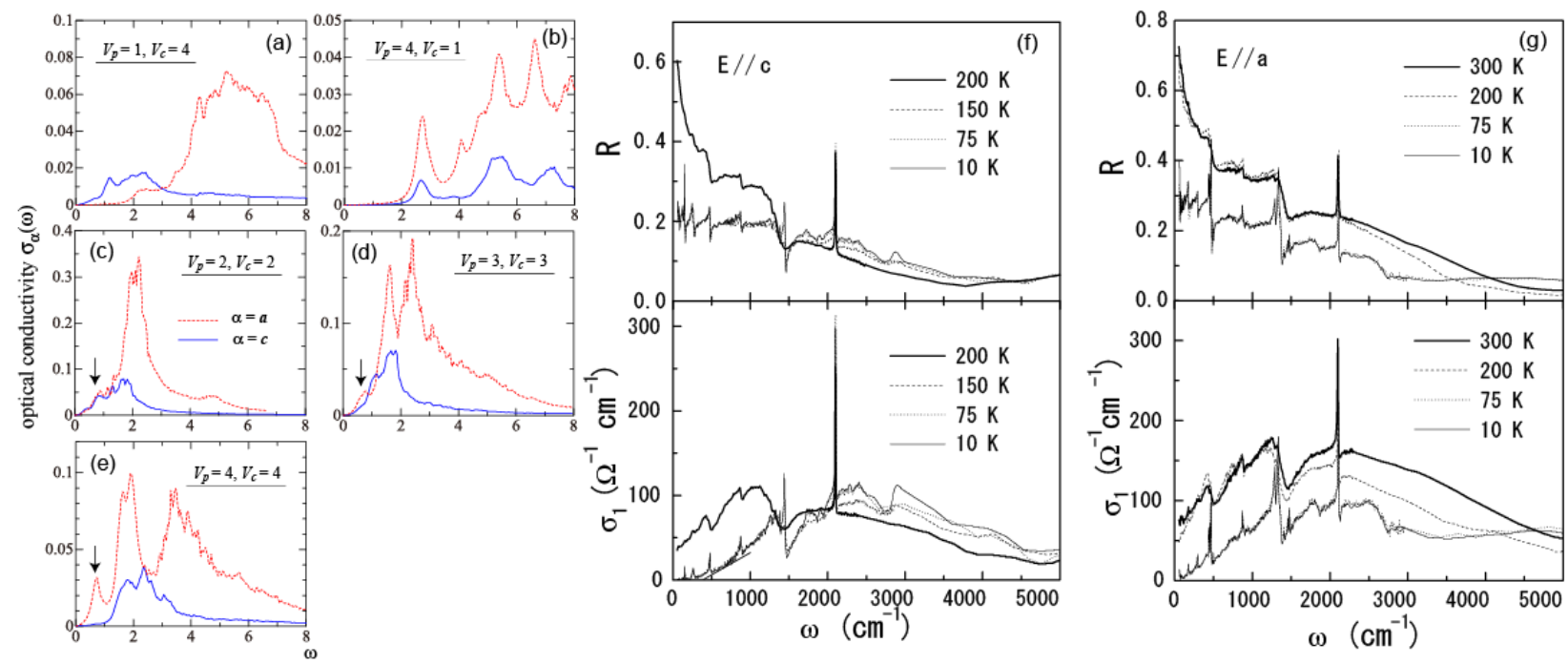
Other compounds with $\varphi>111^{\circ}$ also show similar $\tau \Delta v_{2}$ » 1 spectra with a broad linewidth as shown in Figure 14b,c [97]. As the dihedral angle increases, the $v_{2 \mathrm{P}}$ mode, the counterpart of $v_{2 \mathrm{R}}$, appears more clearly. In addition, $v_{3}$ mode in the low-frequency region of the broad $v_{2 R}$ is more enhanced above $T_{\mathrm{CO}}$ (See Figure 14b). In particular, in $\theta-\mathrm{Cu}_{2}(\mathrm{CN})\left[\mathrm{N}(\mathrm{CN})_{2}\right]_{2}$, another broad $v_{3}$ mode is found at $\sim 1390 \mathrm{~cm}^{-1}$ (not shown) above $T_{\mathrm{CO}}$, when a $780 \mathrm{~nm}$ excitation laser is used [57]. The observation of the precursor band $v_{3}$ means that short-range ordered horizontal and diagonal stripes grow more extensively toward $T_{\mathrm{CO}}$. Actually, x-ray diffuse scattering is observed at $\boldsymbol{q}_{2}=1 / 2 c^{*}$ above $T_{\mathrm{CO}}=220 \mathrm{~K}$ in $\theta-\mathrm{Cu}_{2}(\mathrm{CN})\left[\mathrm{N}(\mathrm{CN})_{2}\right]_{2}$ [57]. Although no diffuse scattering has been found in $\theta_{\mathrm{m}}-\mathrm{TlZn}$, the vibronic band of $v_{3}$ is clearly found in the optical conductivity as shown in Figure 15a. Differently from $\theta-\operatorname{RbZn}$, the fluctuation of the threefold lattice is absent, whereas the short-range $2 c$ superlattice is dominant. Watanabe explained this trend theoretically as the result of $t_{d} / t_{p}$ [78], which decreases from positive value $(\sim 0.5)$ to a negative value $(-0.5)$ according to the increase in the dihedral angle in the phase diagram of the $\theta$-type BEDT-TTF salts $[98,99]$. In these two compounds, the frustration with threefold $\mathrm{CO}$ seems to be reduced more than in $\theta-\mathrm{RbZn}$ and $\theta_{\mathrm{o}}-\mathrm{TlZn}$. This may be related to the continuous transformation of the Raman and optical conductivity spectra and the resistivity of $\theta_{\mathrm{m}}$-TlZn and $\theta$ $\mathrm{Cu}_{2}(\mathrm{CN})\left[\mathrm{N}(\mathrm{CN})_{2}\right]_{2}$ in contrast to $\theta-\mathrm{RbZn}$ and $\theta_{\mathrm{o}}-\mathrm{TlZn}$ (See Figures 12,15).

Figure 14. Comparison of the Raman spectra of (a) the CO state of $\theta$-(BEDT-TTF $)_{2}$ $\mathrm{RbZn}(\mathrm{SCN})_{4}$; (b) high-temperature phase of $\theta-(\mathrm{BEDT}-\mathrm{TTF})_{2} \mathrm{Cu}_{2} \mathrm{CN}\left[\mathrm{N}(\mathrm{CN})_{2}\right]_{2}$; and the metallic phase of (c) $\theta_{\mathrm{m}}-(\mathrm{BEDT}-\mathrm{TTF})_{2} \mathrm{TlZn}(\mathrm{SCN})_{4} ;$; d $\theta-(\mathrm{BEDT}-\mathrm{TTF})_{2} \mathrm{RbZn}(\mathrm{SCN})_{4}$; (e) $\theta$-(BEDT-TTF $)_{2} \operatorname{CsZn}(\mathrm{SCN})_{4}$; and (f) $\theta$-(BEDT-TTF $)_{2} \mathrm{I}_{3}$. Note that the $v_{2}$ mode is split in (a), (b), (c), and (d), while $v_{2}$ is merged in (e) and (f). The assignments in $\theta$-(BEDT-TTF) $)_{2}$ $\mathrm{MZn}(\mathrm{SCN})_{4}(\mathrm{M}=\mathrm{Rb}, \mathrm{Cs})$ in the metallic phase are conducted with the aid of ${ }^{13} \mathrm{C}$-substituted compounds [94].

(a) $\theta-\operatorname{RbZn} 20 \mathrm{~K}$

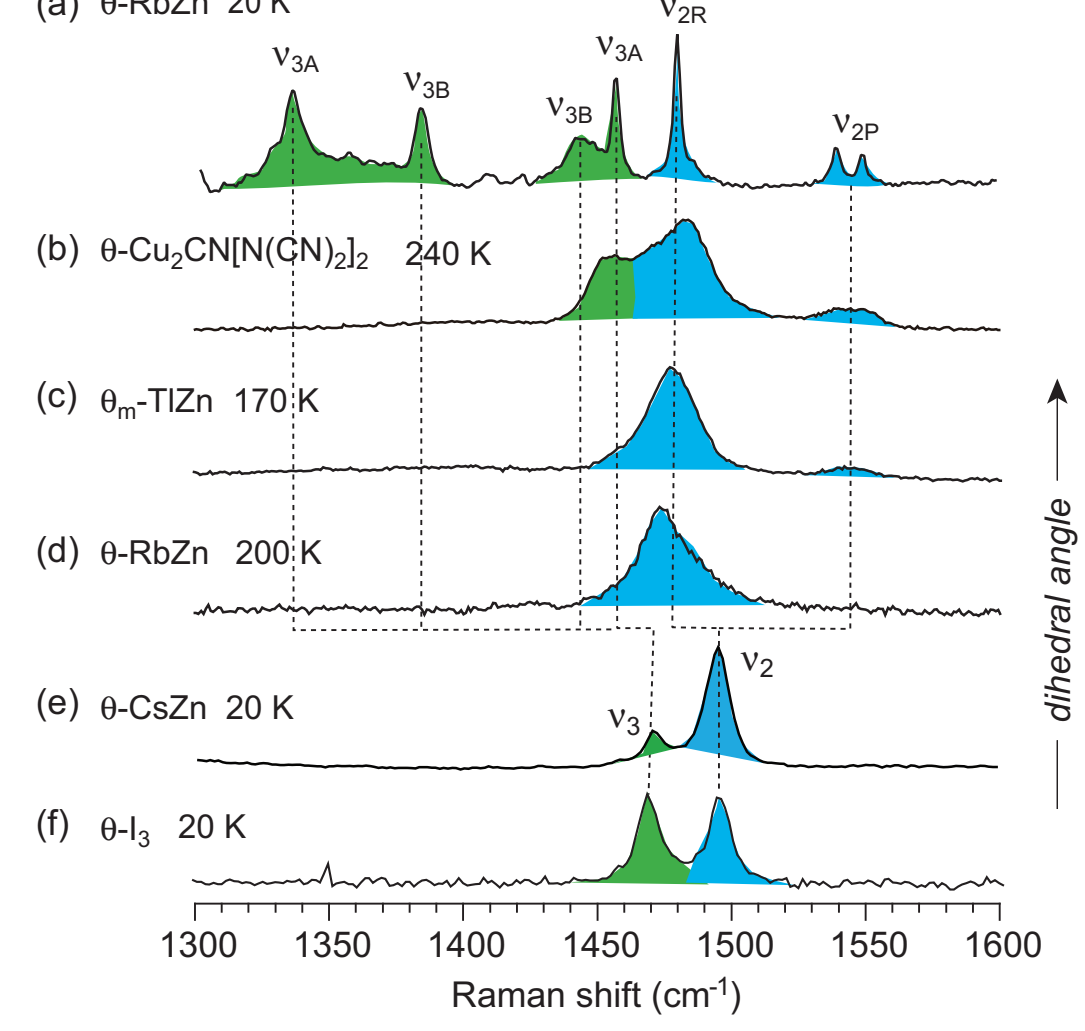


Figure 15. Optical conductivity of (a) $\theta_{\mathrm{m}}-(\mathrm{BEDT}-\mathrm{TTF})_{2} \mathrm{TlZn}(\mathrm{SCN})_{4}$ and (b) $\theta_{\mathrm{o}}-(\mathrm{BEDT}-$ $\mathrm{TTF})_{2} \mathrm{TlZn}(\mathrm{SCN})_{4}$. Note that the spectral variation is discontinuous in $\theta_{\mathrm{o}}-(\mathrm{BEDT}-\mathrm{TTF})_{2}$ $\mathrm{TlZn}(\mathrm{SCN})_{4}$, whereas it is continuous in $\theta_{\mathrm{m}}-(\mathrm{BEDT}-\mathrm{TTF})_{2} \mathrm{TlZn}(\mathrm{SCN})_{4}$. The arrow denotes the vibronic band of $v_{3}$.
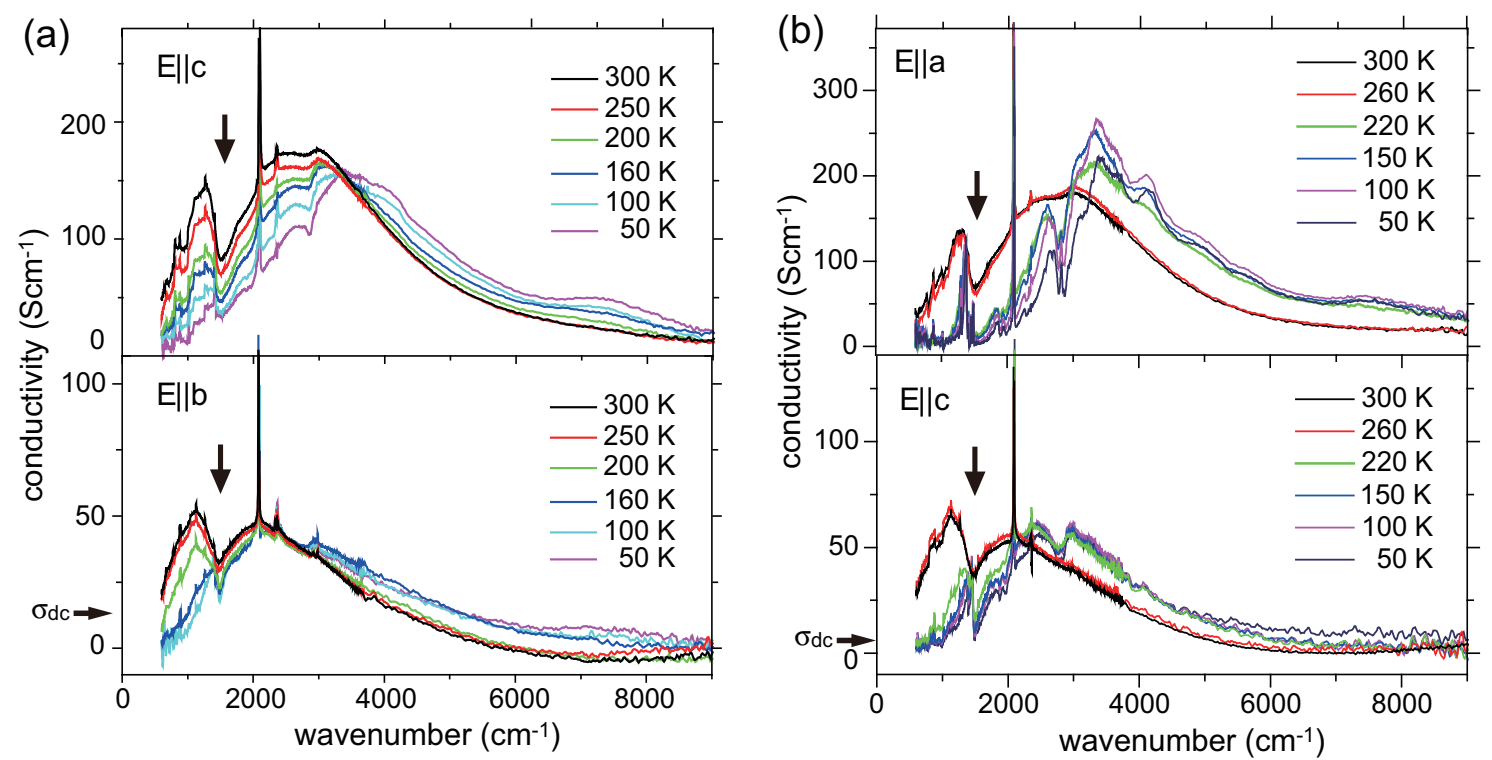

\section{3. $\theta-(B E D T-T T F)_{2} X\left(X=C s Z n(S C N)_{4}, I_{3}\right)$}

The $\theta$-CsZn salt is located at the boundary between the metallic $\theta-\mathrm{I}_{3}$ salt and the large $\varphi$ salts that undergo CO transition. The x-ray diffuse rods are found at $\boldsymbol{q}_{1}=(2 / 3, k, 1 / 3)$ and $\boldsymbol{q}_{2}=(0, k, 1 / 2)$ below $120 \mathrm{~K}[100,101]$. This finding implies that a short-range ordered $3 a \times 3 c$ superlattice (fluctuation of threefold $\mathrm{CO}$ ) coexists with the $2 c$ superlattice (fluctuation of stripe $\mathrm{CO}$ ). The $2 c$ lattice is regarded as the fluctuation of the horizontal stripe which is found in $\theta-\mathrm{RbZn}$ below $T_{\mathrm{CO}}$. On lowering the temperature, the intensity of $\boldsymbol{q}_{2}$ increases as the resistivity increases from about $140 \mathrm{~K}$. Although the correlation length of the $2 c$ lattice grows up on lowering the temperature, it does not attain a long-range order. Interestingly, an external current suppresses the $\boldsymbol{q}_{2}$ domain at $12 \mathrm{~K}$ keeping the $\boldsymbol{q}_{1}$ domain intact [100]. This finding is related to the nonlinear conduction [102] and the thyristor effect [103]. The ${ }^{13} \mathrm{C}$-NMR study argued the following points. (1) Remarkable charge disproportionation with extremely slow fluctuations is observed in the temperature range of $294 \mathrm{~K}-140 \mathrm{~K}$; (2) The short-range $\mathrm{CO}$ with the amplitude of $\Delta \rho \sim 0.4-0.6$ becomes almost static in the region of $140 \mathrm{~K}-50 \mathrm{~K}$; (3) The amplitude of the CO fluctuation becomes very much reduced below $30 \mathrm{~K}$ [104]. The dielectric permittivity study proposes a glass-like state at low-temperature which consists of conglomerate domains of short-range ordered threefold and stripe CO [105]. Several theoretical studies have been conducted to explain the coexistent and/or frustrating $\mathrm{CO}$ fluctuation using a triangular lattice model $[76,78,96,106]$. The stripes and non-stripe threefold $\mathrm{CO}$ are competing near an isotropic triangular lattice $\left(V_{c} \sim V_{p}\right)$, forming a charge-ordered metal $[78,96]$. The origin of the threefold $\mathrm{CO}$ is the nesting of the Fermi surface, while the origin of the stripe $\mathrm{CO}$ is the off-site Coulomb interaction [106], or both instabilities come from the Fermi surface nesting [76]. In all cases, there is a wide metallic 
region due to the frustration between the stripe and threefold $\mathrm{CO}$ fluctuation near the isotropic triangular lattice.

Figure 16 shows the Raman spectra of $\theta-C s Z n$ and $\theta-\mathrm{I}_{3}$ along with the electrical resistivity of $\theta$-CsZn [94]. $\theta-(\mathrm{ET})_{2} \mathrm{I}_{3}$ is metallic down to low temperature and undergoes a superconducting transition at $3.6 \mathrm{~K}[107,108]$. In both compounds, $v_{2}$ appears as a single peak near the frequency of $\rho=0.5$ [46] over the whole temperature range. Different from $\theta-\mathrm{I}_{3}$, a very broad vibronic mode is observed at $1200-1300 \mathrm{~cm}^{-1}$ in $\theta-C s Z n$ as shown in Figure 16. The Raman cross section of this vibronic mode is enhanced when charge disproportionation is enlarged as shown in Figure $2 b$ (Section 2). This observation suggests that the correlation length of the CO fluctuation grows upon decreasing the temperature, since the intensity of the vibronic band increases. From the polarization dependence, $v_{3 \mathrm{~A}}$ and $v_{3 B}$ are respectively classified into $A$ and $B$ species in the factor group $C_{2}$ [94]. This result means that the short-range CO domain has a screw axis symmetry. Therefore, the $2 c$ superlattice with the horizontal stripe seems to contribute to this vibronic mode [109]. This observation is consistent with $\mathrm{x}$-ray diffuse scattering that shows the evolution of the short-range ordered $2 c$ superlattice. Although threefold CO fluctuation is observed in both $\theta-C s Z n$ and $\theta-R b Z n$, the features of $v_{2}$ of $\theta-C s Z n$ are very different from that of $\theta-\mathrm{RbZn}$. The $v_{2}$ mode of $\theta-\mathrm{CsZn}$ appears as a slightly broad single band at the frequency of $\rho \sim 0.5$, whereas the $v_{2}$ mode of $\theta-R b Z n$ appears as a much broader band at the frequency of the charge-rich site. The infrared-active mode $v_{27}$ also appears in the same way as $v_{2}$ [94]. This result means that the product of the correlation time and splitting width is smaller than unity, $\tau \Delta v_{2}<1$, in $\theta$-CsZn (See Figure $4 \mathrm{a}, \Delta=\Delta v_{2} / 2$ ), whereas $\tau \Delta v_{2}>1$ in $\theta-\mathrm{RbZn}$ as described in Section 4.2. Therefore, the correlation time and/or CO amplitude of $\theta-\mathrm{CsZn}$ is much smaller than that of $\theta-\mathrm{RbZn}$. If the fluctuation is almost static on the time scale of vibrational spectroscopy as suggested by NMR [104], $\Delta v_{2}$ is dominated by spatial inhomogeneity. The linewidth is about $10 \mathrm{~cm}^{-1}$ below $100 \mathrm{~K}$. If we assume a static Gaussian distribution for the site charge, the amplitude is roughly estimated as $\Delta \rho<0.08$. This small amplitude is inconsistent with the argument of ${ }^{13} \mathrm{C}-\mathrm{NMR}$ [104]. However, the small amplitude is consistent with the much weaker intensity (diffuse/Bragg $\sim 10^{-5}$ ) of the diffuse scattering in $\theta$-CsZn [100] compared with that (diffuse/Bragg $\sim 10^{-3}$ ) in $\theta-\mathrm{RbZn}$ [91]. The small amplitude seems to be attributed mainly to the strong frustration between the threefold and stripe fluctuation. Seo demonstrated that in such a case, both peaks in the charge correlation function $C(\boldsymbol{q}, \omega)$ corresponding to stripe and threefold fluctuation have higher excitation energy compared to the nonfrustrate system [41]. This result implies that the amplitude of CO cannot grow when they are competing.

The optical conductivity of $\theta-\mathrm{CsZn}$ is shown in Figure 17 [94]. Compared with the optical conductivity of $\theta-\operatorname{RbZn}[95]$, the spectral weight of $\theta-\mathrm{CsZn}$ is located in the low-frequency region, which is similar to the optical conductivity of $\beta^{\prime \prime}-(\mathrm{ET})(\mathrm{TCNQ})$ shown in Figure 8 . Since the conductivity should approach the dc conductivity shown in Figure 17, the peak which corresponds to $\sigma_{\mathrm{IQ}}$ is expected below $600 \mathrm{~cm}^{-1}$, and the Drude response is likely to be missing. This speculation is consistent with the optical conductivity reported by Wang [110], wherein a strong peak accompanied by several vibronic bands appears below $600 \mathrm{~cm}^{-1}$ without Drude response. The $V / t$ dependence of the optical conductivity similar to $\beta^{\prime \prime}$-(ET)(TCNQ) has been theoretically calculated for a triangular lattice [96]. The dips at $1300 \mathrm{~cm}^{-1}$ and $877 \mathrm{~cm}^{-1}$ are the vibronic modes respectively corresponding to $v_{3}$ and $v_{7}$, which arise from the EMV interaction. These vibronic modes are forbidden in the average 
structure of $\theta-\mathrm{CsZn}$. The appearance of these vibronic modes also strongly indicates structural fluctuations, which probably corresponds to the $3 a \times 3 c$ and $2 c$ short-range superlattices.

Figure 16. Temperature dependence of the Raman spectra of $\theta-(B E D T-T T F)_{2} I_{3}$ and $\theta$-(BEDT-TTF $)_{2} \mathrm{CsZn}(\mathrm{SCN})_{4}$ along with the electrical resistivity of $\theta$-(BEDT-TTF $)_{2}$ $\mathrm{CsZn}(\mathrm{SCN})_{4}$. The inset shows the temperature dependence of the linewidth of the $v_{2}$ mode. The Raman spectra of $\theta-(\mathrm{BEDT}-\mathrm{TTF})_{2} \mathrm{I}_{3}$ and $\theta-(\mathrm{BEDT}-\mathrm{TTF})_{2} \mathrm{CsZn}(\mathrm{SCN})_{4}$ are modified from Figure 2c of ref. [111] and Figure 3 of ref. [94], respectively.
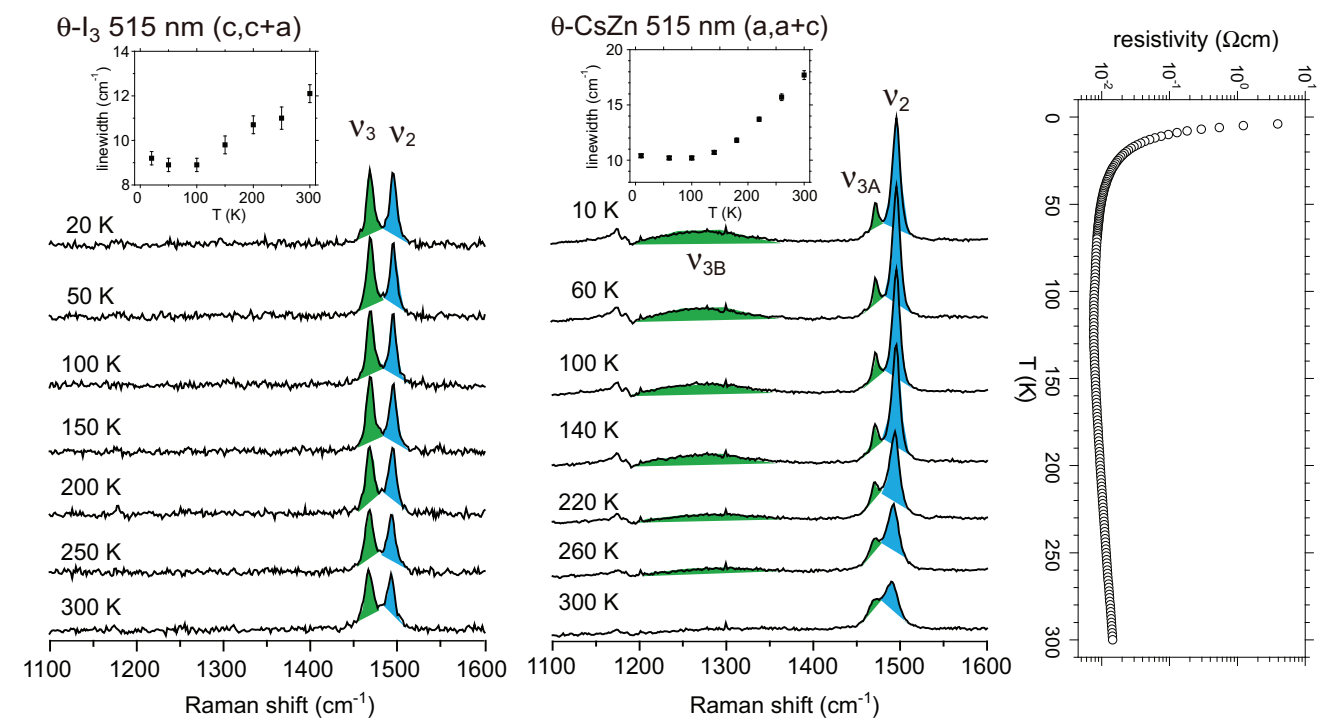

Figure 17. Optical conductivity of $\theta-(\mathrm{BEDT}-\mathrm{TTF})_{2} \mathrm{CsZn}(\mathrm{SCN})_{4}$. Blue colored circles in the bottom panel show the dc conductivity values. The arrows show the vibronic modes appearing as dips. The color of the E $\| c$ spectrum denotes the same temperature as that of the E $\| a$ spectrum.
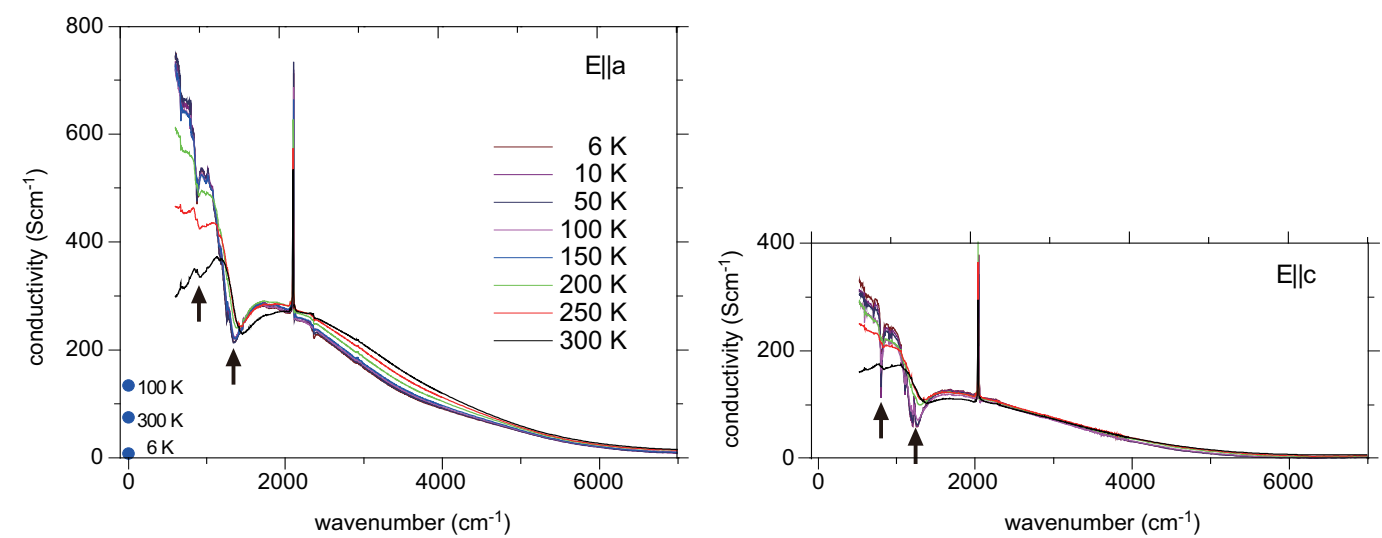

The dihedral angle of $\theta-\mathrm{I}_{3}$ is the largest among the $\theta$-type BEDT-TTF salts, and the fluctuation of $\mathrm{CO}$ is suppressed in $\theta-\mathrm{I}_{3}$ [78]. If we estimate the amplitude in the same way as for $\theta-\mathrm{CsZn}$, the amplitude is estimated as $\Delta \rho<0.07$. Actually the $v_{2}$ mode appears as a single band and the vibronic band is not found in the Raman spectrum of $\theta-\mathrm{I}_{3}$ as shown in the left panel of Figure 16. Takenaka et al., reported the optical conductivity in the frequency range of $80-48000 \mathrm{~cm}^{-1}$ [112]. As the bandwidth is widest among the $\theta$-type BEDT-TTF salts, the spectral weight of the optical conductivity in $\theta-\mathrm{I}_{3}$ is 
located in the frequency region lower than that of $\theta-\mathrm{CsZn}$. As is the case of $\beta^{\prime \prime}$-(BEDT-TTF)(TCNQ), the collapse of the coherent quasiparticle state accompanying the high-frequency shift of the spectral weight is observed upon increasing the temperature. The sublattice of BEDT-TTF in $\theta-\mathrm{I}_{3}$ is described by the orthorhombic cell which is the same as the average unit cell of $\theta$-CsZn [113]. However, a weak vibronic mode is found at $\sim 1410 \mathrm{~cm}^{-1}$ as a dip $[112,114]$. This vibronic band might suggest structural fluctuation perhaps associated with charge order.

\section{5. $\alpha$-(BEDT-TTF) $)_{2} \mathbf{I}_{3}$ and Related Compounds}

Among the various known organic conductors [115-118], $\alpha$-(BEDT-TTF $)_{2} \mathrm{I}_{3}$ (abbreviated as $\alpha-\mathrm{I}_{3}$ ) shows rich electronic properties such as charge ordering [119], superconductivity [120], zero-gap state (ZGS) [121,122], photo-induced phase transition [123], and nonlinear optical response [124].The crystal structure of $\alpha-\mathrm{I}_{3}$ consists of alternating layers of the anion and donor [125]. The donor layer has a herringbone arrangement of BEDT-TTF molecules. The unit cell accommodates two holes, which are distributed in four BEDT-TTFs named A, A', B, and $\mathrm{C}$. At room temperature, $\mathrm{A}$ and $\mathrm{A}^{\prime}$ are connected by inversion symmetry, and $\mathrm{B}$ and $\mathrm{C}$ are located on the inversion center. $\alpha-\mathrm{I}_{3}$ exhibits a first-order metal-insulator (MI) phase transition at $T_{\mathrm{MI}}=135 \mathrm{~K}$ [125]. Kino and Fukuyama examined the effect of on-site Coulomb interaction within the Hartree-Fock approximation, and they predicted that a charge localization with a vertical stripe is the cause of the MI transition [13,126]. Before the theoretical prediction, Moldenhauer et al., reported that the localization of electrons occurs at the B and $\mathrm{C}$ sites whereas the electrons at the $\mathrm{A}$ and $\mathrm{A}^{\prime}$ sites are delocalized below $\mathrm{T}_{\mathrm{MI}}[12]$. The ${ }^{13} \mathrm{C}-\mathrm{NMR}$ study suggested the existence of two differently charged molecules below $\mathrm{T}_{\mathrm{MI}}[119,127]$. Seo introduced intersite Coulomb interaction $V$, and he proposed that the localized charge would form a horizontal stripe [16]. These two models can be distinguished by the symmetry consideration, since the vertical stripe is compatible with the space group $P \overline{1}$, whereas the horizontal stripe requires $P 1$. Raman spectroscopy was used to examine the selection rule of the charge-sensitive mode and the symmetry reduction from $P \overline{1}$ to $P 1$ below $\mathrm{T}_{\mathrm{MI}}$ was claimed [111]. An x-ray diffraction study supported $P 1$, and showed a horizontal stripe structure [128]. Decisive evidence for the breaking of inversion symmetry was given by the experiment of second harmonic generation (SHG) [124]. Recently, Alemany et al., pointed out that the hydrogen bond between the ethylene groups of BEDTTTF and $\mathrm{I}_{3}{ }^{-}$plays an essential role in the CO transition of $\alpha-\mathrm{I}_{3}$ [129].

In the MI transition of $\alpha-I_{3}$, Raman [111] and infrared [130] spectra showed drastic changes as seen in Figure 18. The assignment of these bands along with vibronic bands was conducted with the aid of

${ }^{13} \mathrm{C}$ and deuterium substituted compounds. The $\mathrm{CO}$ and metallic phases will be discussed respectively in Sections 5.1 and 5.2 along with the detailed assignment. Unlike $\theta$-type BEDT-TTF salts, it has been reported for $\alpha-\mathrm{I}_{3}$ that the site-charge distribution is non-uniform in the metallic phase $[13,111,128]$, although the average hole number per site is 0.5 in the case of uniform distribution. The non-uniform site charge distribution of metallic $\alpha-\mathrm{I}_{3}$ will be discussed along with the isostructural metallic $\alpha$-(BEDT-TTF $)_{2} \mathrm{NH}_{4}(\mathrm{SCN})_{4}$ in Section 5.3. One of the most attractive properties of $\alpha-\mathrm{I}_{3}$ is the ferroelectric properties in the $\mathrm{CO}$ state. In this context, another herringbone type compound $\alpha^{\prime}-\mathrm{IBr}_{2}$ will discussed in Section 5.4. 
Figure 18. (a) Temperature dependence of the Raman spectrum of $\alpha$-(BEDT-TTF $)_{2} \mathrm{I}_{3}$. The $v_{2}$ and $v_{3}$ modes are shown as blue and green, respectively. A drastic spectral change is observed below $120 \mathrm{~K}$. The difference in the transition temperature comes from the laser heating effect; (b) Temperature dependence of the infrared spectrum of $\alpha$-(BEDT-TTF) $)_{2} \mathrm{I}_{3}$, which was obtained by the Kramer-Kronig transformation of the reflectivity polarized along the c-axis (perpendicular to the conducting plane). The $6 \mathrm{~K}$ spectrum drawn by the dashed line is the optical conductivity of the deuterium substituted compound, $\alpha-\left(\mathrm{d}_{8} \text {-BEDT-TTF }\right)_{2} \mathrm{I}_{3}$; (c) Electrical resistivity normalized by room temperature value. The optical conductivity is taken from Figure 1 of ref. [130].

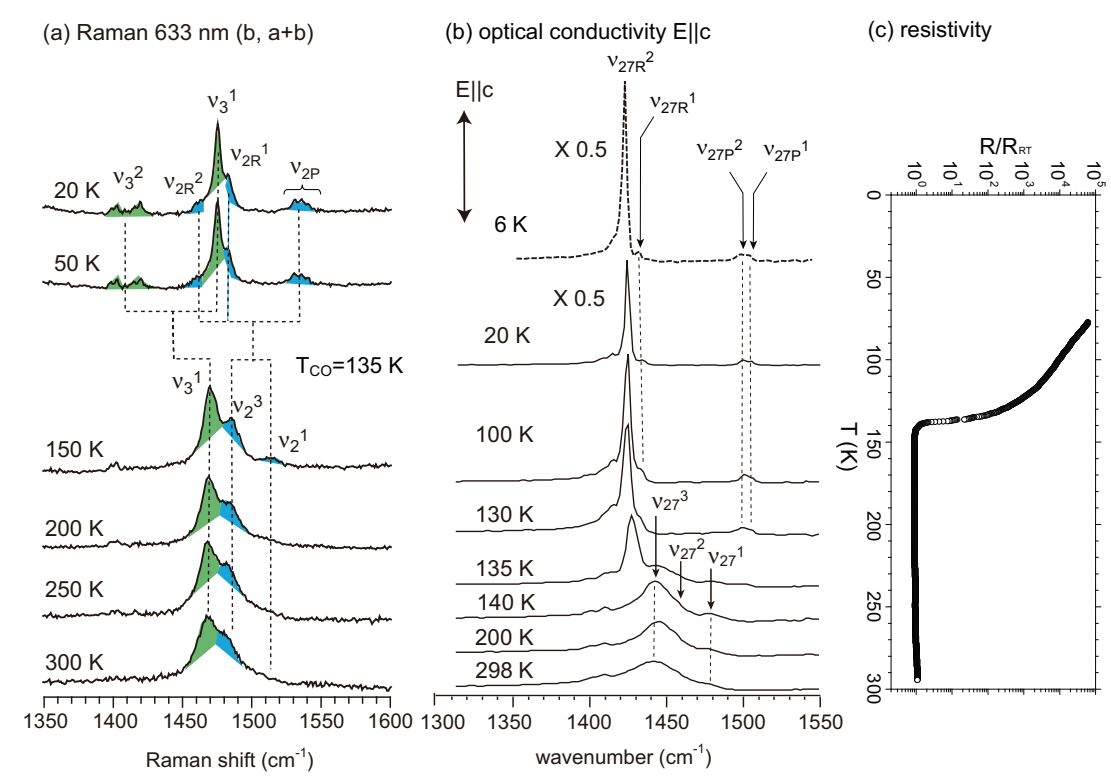

\subsection{Charge-Ordered Phase of $\alpha-(B E D T-T T F){ }_{2} I_{3}$}

Figure 19 shows infrared and Raman spectra of $\alpha-\mathrm{I}_{3}, \alpha-\left(\mathrm{d}_{8}-\mathrm{BEDT}-\mathrm{TTF}\right)_{2} \mathrm{I}_{3}$, and $\alpha-\left({ }^{13} \mathrm{C}-\mathrm{BEDT}-\mathrm{TTF}\right)_{2} \mathrm{I}_{3}$, all in the $\mathrm{CO}$ state. In this spectral region, BEDT-TTF has three $\mathrm{C}=\mathrm{C}$ stretching modes, $v_{2}, v_{3}$, and $v_{27}$ which are shown in Figure 1, and bending modes of ethylene groups. The assignment of the Raman-active modes is the same as in ref. [111], except for the Raman bands at $1476 \mathrm{~cm}^{-1}$ and $1462 \mathrm{~cm}^{-1}$, which were assigned to $v_{3}{ }^{1}$ and $v_{2 \mathrm{R}}{ }^{2}$, respectively. These are interchanged in Table 1 , as the isotope shift of $v_{2}$ and $v_{3}$ became closer to each other within each mode. Strictly speaking, however, this kind of assignment makes no sense, as these two modes are strongly mixed with each other. The vibronic modes in the conducting plane are assigned with the aid of isotope shift and comparison with the Raman spectrum. The very broad band, wherein the peak was different between the $\mathrm{E} \| a$ and $\mathrm{E} \| b$ spectra, was assigned to $v_{3}{ }^{4}$, since the frequency of the vibronic mode depended upon the electronic excitation spectrum. The vibrational $v_{27}$ mode is observable in the optical conductivity spectrum polarized along the $c$-axis. Some bending modes of ethylene group are overlapped in the region of the $v_{27 \mathrm{R}}$ modes (See Table 1 and Figure $\left.19 \mathrm{a}\right)$. Therefore, the spectrum of $\alpha-\left(\mathrm{d}_{8} \text {-BEDT-TTF }\right)_{2} \mathrm{I}_{3}$ is necessary for the assignment of $v_{27 R}$. The large dip at $\sim 2690 \mathrm{~cm}^{-1}$ appearing in the high-frequency region of the optical conductivity (not shown) is interpreted by Yamamoto as the Fano anti-resonance due to the 
vibronic overtone of $v_{3}$ [131]. Recently, Yamamoto et al., have discussed the relationship between the vibronic overtone and optical nonlinearity and ferroelectricity [132].

Figure 19. (a) Optical conductivity polarized along the $b$ and $a$ directions, Raman spectra of $\alpha$-(BEDT-TTF $)_{2} \mathrm{I}_{3}$, and optical conductivity polarized along the $c$ direction; (b) Optical conductivity polarized along the $b$ and $a$ directions and Raman spectra of $\alpha-\left({ }^{13} \mathrm{C}-\mathrm{BEDT}-\mathrm{TTF}\right)_{2} \mathrm{I}_{3}$.

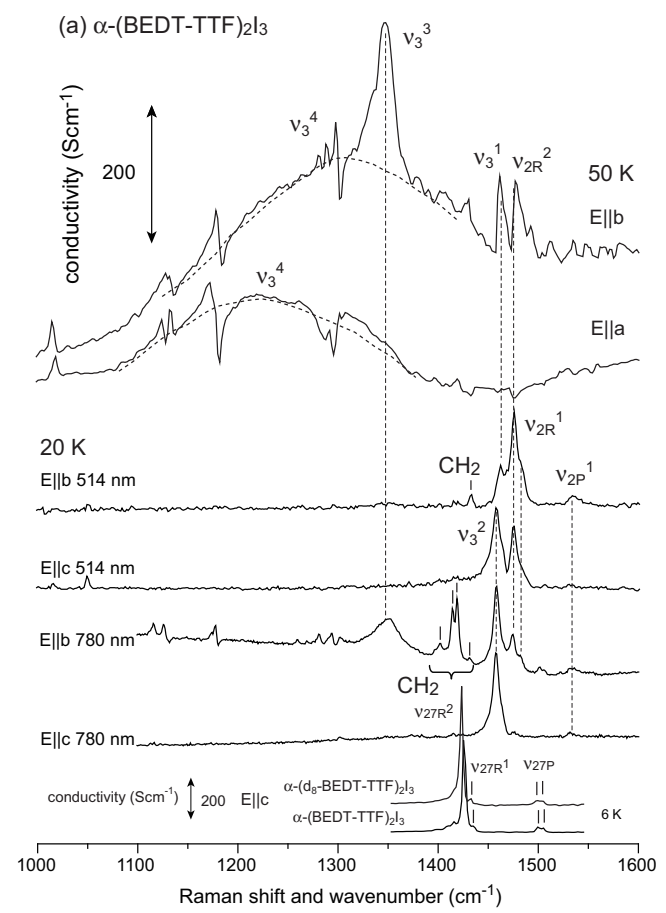

$$
\text { (b) } \alpha-\left({ }^{13} \mathrm{C}-\mathrm{BEDT}-\mathrm{TTF}\right)_{2} \mathrm{l}_{3}
$$

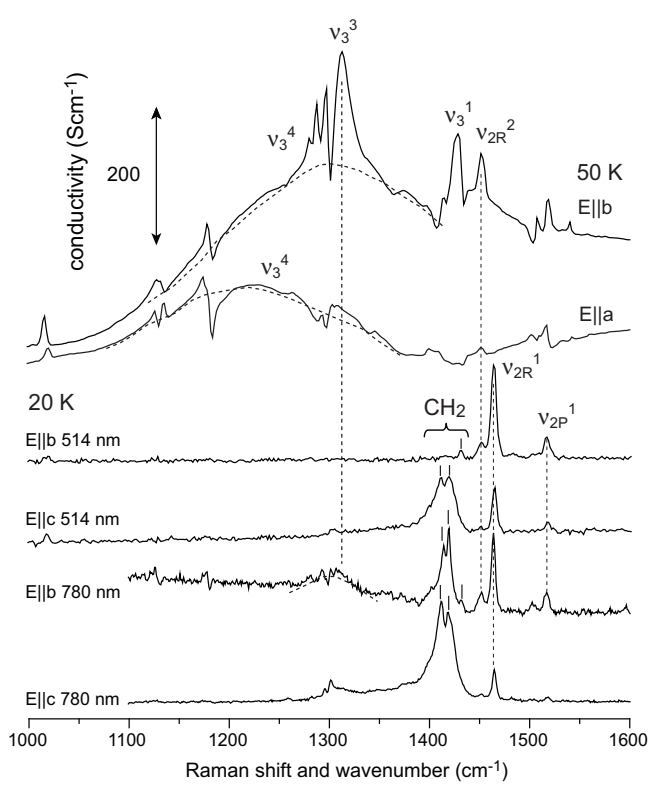

Table 1. Assignment of the infrared and Raman modes of $\alpha-(\text { BEDT-TTF })_{2} \mathrm{I}_{3}$. The $\Delta v$ values show the isotope shift, $\Delta v_{\mathrm{j}}=v_{\mathrm{j}}^{12}-v_{\mathrm{j}}^{13}$.

\begin{tabular}{|c|c|c|c|c|c|c|}
\hline & \multicolumn{3}{|c|}{ Raman (20 K) } & \multicolumn{3}{|c|}{ Infrared (50 K) } \\
\hline & $\alpha-I_{3}$ & $\alpha-\left({ }^{13} C\right)_{2} I_{3}$ & $\Delta v$ & $\alpha-I_{3}$ & $\alpha-\left({ }^{13} C\right)_{2} I_{3}$ & $\Delta v$ \\
\hline $\operatorname{dip}\left(2 v_{3}{ }^{3}\right)$ & - & - & - & 2688 & 2628 & 60 \\
\hline$v_{2 P}{ }^{1}$ & 1536 & 1517 & 19 & & (1518?) & - \\
\hline$v_{2 \mathrm{P}}^{2}$ & - & - & - & - & (1508?) & - \\
\hline$v_{2 R}{ }^{1}$ & 1483 & 1465 & 18 & - & - & - \\
\hline$v_{2 R}^{2}$ & 1476 & 1452 & 24 & 1477 & 1454 & 23 \\
\hline$v_{3}^{1}$ & 1462 & - & - & 1462 & 1431 & 31 \\
\hline$v_{3}^{2}$ & 1458 & - & - & - & - & - \\
\hline$v_{3}^{3}$ & 1349 & 1315 & 34 & 1346 & 1315 & 31 \\
\hline \multirow[t]{3}{*}{$v_{3}{ }^{4}$} & (2) & - & - & $\sim 1300(\mathrm{E} \| b)$ & $\sim 1300(\mathrm{E}|| b)$ & - \\
\hline & & & & $\sim 1230(\mathrm{E} \| a)$ & $\sim 1230(\mathrm{E} \| a)$ & \\
\hline & $\int 1431$ & 1431 & - & - & - & - \\
\hline $\mathrm{CH}_{2^{-}}$ & 1419 & 1420 & - & - & - & - \\
\hline \multirow[t]{2}{*}{ Bending } & 1414 & 1414 & - & - & - & - \\
\hline & 1402 & 1402 & - & - & - & - \\
\hline$v_{27 P}{ }^{1}$ & - & - & - & $1506(\mathrm{E} \| \mathrm{c})$ & $1505(\mathrm{E} \| \mathrm{c})$ & - \\
\hline$v_{27 P^{2}}$ & - & - & - & $1501(\mathrm{E} \| \mathrm{c})$ & $1500(\mathrm{E} \| \mathrm{c})$ & - \\
\hline$v_{27 R} 1$ & - & - & - & $1435(\mathrm{E} \| \mathrm{c})$ & $1432(\mathrm{E} \| \mathrm{c})$ & - \\
\hline$v_{27 R^{2}}$ & - & - & - & $1425(\mathrm{E} \| \mathrm{c})$ & $1423(\mathrm{E} \| \mathrm{c})$ & - \\
\hline
\end{tabular}


If the unit cell has inversion symmetry, Raman-active band cannot be observed in an infrared spectrum and vice versa. As shown in Table $1, v_{2 \mathrm{R}}{ }^{2}, v_{3}{ }^{1}$, and $v_{3}{ }^{3}$ are found in both the Raman and infrared spectra, which violate the mutual exclusion rule. As describe above, comparison of the Raman spectrum with the infrared spectrum is a good method to judge the breaking of inversion symmetry. Based on the assignment, it becomes clear that the $v_{2}$ and $v_{27}$ modes are split into two groups, $v_{2 \mathrm{P}}$ and $\left(v_{2 \mathrm{R} 1}, v_{2 \mathrm{R} 2}\right)$ in the Raman spectrum and $\left(v_{27 \mathrm{P}}{ }^{1}, v_{27 \mathrm{P}}{ }^{2}\right)$ and $\left(\mathrm{v}_{27 \mathrm{R}}{ }^{1}, v_{27 \mathrm{R}}{ }^{2}\right)$ in the infrared spectrum as shown in Figure 18. Applying the linear relationship [46] to the $v_{27}$ mode $(50 \mathrm{~K})$, the site charge distribution is estimated as $(0.8,0.7,0.3,0.2)$ [130], which are approximately equal to the values $(0.85,0.80,0.20$, $0.15)$ estimated by Ivek et al. [133]. The CO amplitude $\left(\Delta \rho_{\max }=0.6\right)$ is comparable to that of $\theta-\mathrm{RbZn}$. Kakiuchi et al., examined the breakdown of Friedel's law by anomalous scattering effect, and they concluded a symmetry reduction from $P \overline{1}$ to $P 1$ [128]. Using the space group $P 1$, they determined the molecular geometry of each site, and provided consistent result for the site charge distribution as $\rho_{\mathrm{A}}=0.82(9), \rho_{\mathrm{B}}=0.73(9), \rho_{\mathrm{A}},=0.29(9), \rho_{\mathrm{C}}=0.26(9)$ at $20 \mathrm{~K}$. Recently, Alemany et al., reported the site charges as $\rho_{\mathrm{A}}=0.638, \rho_{\mathrm{B}}=0.577, \rho_{\mathrm{A}^{\prime}}=0.438, \rho_{\mathrm{C}}=0.359$ using a numerical atomic orbital density functional theory (DFT) approach [129]. This CO amplitude $\rho_{\max }=0.28$ is significantly smaller than the experimentally estimated value of $\rho_{\max }=0.56-0.70$. The site charge distribution has been estimated from the ${ }^{13} \mathrm{C}$-NMR shift, which consists of Knight shift and chemical shift [15,134]. The chemical shift reflects the local charges, whereas the Knight shift is proportional to the local spin susceptibility which reflects the density of states at the Fermi level [135]. Therefore, the chemical shift should be analyzed to estimate the site charges. Kawai and Kawamoto conducted a ${ }^{13} \mathrm{C}-\mathrm{NMR}$ study using a single side ${ }^{13} \mathrm{C}$-enriched molecule [135]. To avoid the effect of the Knight shift and ring current within the molecule, they applied a magnetic field nearly parallel to the long axis of the molecule in the non-magnetic state (at $60 \mathrm{~K}$ ). They determined the chemical shift tensor of the four sites, and estimated the site charge to be $\rho_{\mathrm{R}} \sim 0.74$ and $\rho_{\mathrm{P}} \sim 0.19$. If we simply apply the relation between the chemical shift and site charge which they present, site charge distribution is estimated as $\rho_{\mathrm{A}}=0.74, \rho_{\mathrm{B}}=0.76$, $\rho_{\mathrm{A}}{ }^{\prime}=0.18$, and $\rho_{\mathrm{C}}=0.20$. All of these results studied by vibrational spectroscopy, $\mathrm{x}$-ray diffraction, and ${ }^{13} \mathrm{C}$-NMR approximately consistent with each other.

Yamamoto et al., found strong second harmonic generation (SHG) in the CO state, using 4 ps pulse laser with a wavelength of $1.4 \mu \mathrm{m}$ as the fundamental light $[124,136]$. The evolution of the second harmonic light just below $T_{\mathrm{CO}}$ is direct evidence for the breakdown of the inversion symmetry. They determined the nonlinear electric susceptibility $\chi^{(2)}{ }_{\mathrm{ij}}(2 \omega ; \omega, \omega)$, where $i$ and $j$ denote the polarization of the second harmonic light and fundamental light, respectively. The relative values against BBO ( $\beta$-barium borate), which is a representative crystal for nonlinear optics, are $21,8.5,44,31$ for the polarization $a a, a b, b a$, and $b b$. Such a strong susceptibility value suggests that the generated electric dipole in a unit cell is coherently extended over a macroscopic domain, that is, a ferroelectric domain. As in conventional ferroelectrics, the crystal is expected to form a multiple domain structure. Ferroelectric domains with opposite polarization are observed utilizing the experimental technique of SHG interferometry [137]. The domain size is found to be more than $0.2 \mathrm{~mm} \times 0.2 \mathrm{~mm}$, which is almost a single domain. Although polarization reversal by an external electric field has not been realized due to high conductivity and low breakdown field, this compound in the CO state is classified as an unconventional ferroelectrics called electronic ferroelectrics [138]. In conventional ferroelectrics, ionic polarization coming from displacement or ordering of ions plays a role in creating a polar 
domain. On the other hand, in electronic ferroelectrics, ions are replaced by electrons, and electronic polarization by charge order causes a polar domain. To show that the polar domain mainly originated from electrons, Yamamoto demonstrated fast photoresponse where the second harmonics diminish by $50 \%$ within $100 \mathrm{fs}$ and recovers within several tenths of a picosecond after the irradiation of a $100 \mathrm{fs}$ laser. This fast photoresponse was studied by Iwai et al., as a photoinduced insulator-to-metal phase transition. Using mid-infrared pump-probe spectroscopy, they observed the transient reflectivity change in the mid-infrared region $[123,139]$. The decay process depends on the intensity of the light pulse. In the case of weak excitation, one photon produces a microscopic metallic domain consisting of about 100 molecules, which decays quickly. In the case of strong excitation, on the other hand, the microscopic domains aggregate with each other forming a quasi-macroscopic metallic domain, which decays slowly. The recovery of the second harmonics corresponds to this slow decay. Recently, Kawakami et al., uncovered the early dynamic process of the photoinduced phase transition using a $12 \mathrm{fs}$ laser [140].

\subsection{Metallic Phase of $\alpha-(B E D T-T T F)_{2} I_{3}$ and Isostructural Metallic Compounds}

\subsubsection{Assignment in Metallic Phase}

We have shown that charge order is the origin of the splitting of the $v_{2}$ and $v_{27}$ modes. If a metallic compound involves crystallographically non-equivalent sites in the unit cell, the charge-sensitive modes split reflecting the non-equivalent sites. This splitting is also described by Equation (1). Figure 20 shows the Raman spectra of metallic $\alpha-\mathrm{I}_{3}, \alpha$-(BEDT-TTF) ${ }_{2} \mathrm{NH}_{4} \mathrm{Hg}(\mathrm{SCN})_{4}$, (abbreviated as $\alpha-\mathrm{NH}_{4} \mathrm{Hg}$ ) and $\theta-\mathrm{I}_{3}$. The first two compounds are isostructural with each other, while the latter one takes herringbone arrangement with higher symmetry. $\alpha-\mathrm{NH}_{4} \mathrm{Hg}$ is metallic down to low temperature, and shows superconductivity at $1 \mathrm{~K}$ [141]. Similar to $\alpha-\mathrm{I}_{3}$, the unit cell of $\alpha-\mathrm{NH}_{4} \mathrm{Hg}$ involves four ET molecules (see Figure 1 of ref. [142] for the definition of A, A', B, and C). As described in the last section, the unit of the $\alpha$-type compound involves three Raman-active modes and one infrared-active mode for $v_{2}$ and $v_{3}$. In the case of $\alpha-I_{3}$, one $v_{2}$ mode is missing, whereas all of the three appear to be present in ${ }^{13} \mathrm{C}$-substitued compound as shown in Figure 20a, $\mathrm{a}^{\prime}, \mathrm{b}, \mathrm{b}^{\prime}$. From the isotope shift, the three bands in Figure 20a can be safely assigned to $v_{2}{ }^{1}, v_{2}{ }^{2}$, and $v_{3}{ }^{3}$. In the case of $\alpha-\mathrm{NH}_{4} \mathrm{Hg}$, three $v_{2}$ and two $v_{3}$ modes are found and they are assigned based on the isotope shift shown in Figure 20c, $\mathrm{c}^{\prime}$.The remaining Raman-active $v_{3}$ mode is missing from this Raman spectrum. Similar splitting of $v_{27}$ is described in ref. [130]. The crystal of $\theta-\mathrm{I}_{3}$ belongs to the monoclinic system with space group of $P 2_{1} / \mathrm{c}$, and the unit cell contains four BEDT-TTFs. However, the arrangement of BEDT-TTF can be approximately described by the orthorhombic system with space group Pnma with the unit cell accommodating two crystallographically equivalent BEDT-TTFs $[113,143]$. Due to the high symmetry of the unit cell, $v_{2}$ and $v_{3}$ appear as a single band at $\rho \sim 0.5$ as shown in Figure 20d. The frequencies of the $v_{2}$ and $v_{3}$ modes of these compounds are shown in Table 2. 
Figure 20. Raman spectra of $\alpha-(\mathrm{BEDT}-\mathrm{TTF})_{2} \mathrm{I}_{3}$ and $\alpha$-(BEDT-TTF $)_{2} \mathrm{NH}_{4} \mathrm{Hg}(\mathrm{SCN})_{4}$, and the ${ }^{13} \mathrm{C}$-substitued compound, in which two carbon atoms of the central $\mathrm{C}=\mathrm{C}$ bond of BEDT-TTF are substituted by ${ }^{13} \mathrm{C}$. (a) and (b) $\alpha$-(BEDT-TTF $)_{2} \mathrm{I}_{3} ;\left(\mathbf{a}^{\prime}\right)$ and $\left(\mathbf{b}^{\prime}\right) \alpha-\left({ }^{13} \mathrm{C}-\mathrm{BEDT}-\mathrm{TTF}\right)_{2} \mathrm{I}_{3}$; (c) $\quad \alpha-(\mathrm{BEDT}-\mathrm{TTF})_{2} \mathrm{NH}_{4} \mathrm{Hg}(\mathrm{SCN})_{4} ; \quad\left(\mathbf{c}^{\prime}\right) \quad \alpha-\left({ }^{13} \mathrm{C}-\mathrm{BEDT}-\mathrm{TTF}\right)_{2} \mathrm{NH}_{4} \mathrm{Hg}-(\mathrm{SCN})_{4}, \quad$ and (d) $\theta-(\text { BEDT-TTF })_{2} \mathrm{I}_{3}$.

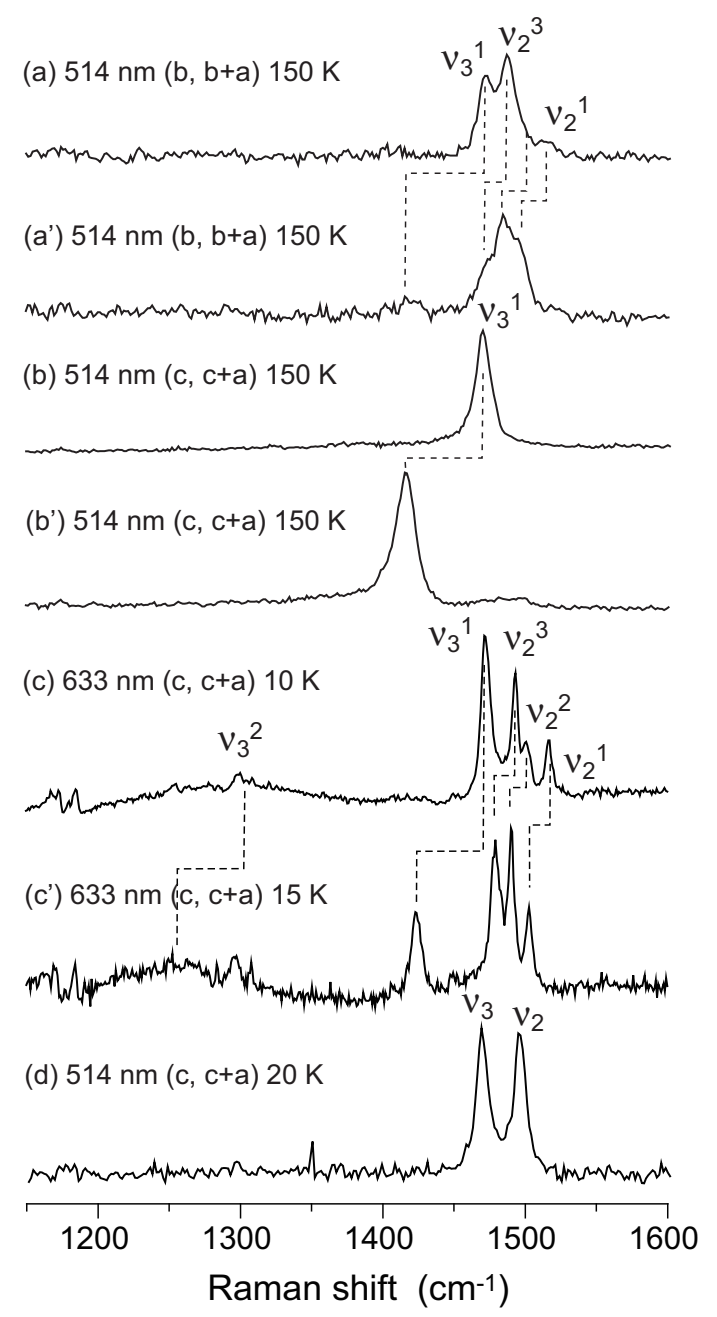

Table 2. Assignment of the Raman modes of $\alpha-(\mathrm{BEDT}-\mathrm{TTF})_{2} \mathrm{I}_{3}, \alpha-(\mathrm{BEDT}-\mathrm{TTF})_{2}$ $\mathrm{NH}_{4} \mathrm{Hg}(\mathrm{SCN})_{4}$, and $\theta-(\mathrm{BEDT}-\mathrm{TTF})_{2} \mathrm{I}_{3}$.

\begin{tabular}{|c|c|c|c|c|c|c|c|}
\hline & $\alpha-\left({ }^{12} \mathrm{C}-\mathrm{ET}\right)_{2} \mathbf{I}_{3}$ & $\left.\alpha-\left({ }^{13} \mathrm{C}-\mathrm{ET}\right)\right)_{2} \mathbf{I}_{3}$ & & $\begin{array}{c}\alpha-\left({ }^{12} \mathrm{C}-\mathrm{ET}\right)_{2} \\
\mathrm{NH}_{4} \mathrm{Hg}(\mathrm{SCN})_{4}\end{array}$ & $\begin{array}{c}\alpha-\left({ }^{13} \mathrm{C}-\mathrm{ET}\right)_{2} \\
\mathrm{NH}_{4} \mathrm{Hg}(\mathrm{SCN})_{4}\end{array}$ & & $\theta-(\mathbf{E T})_{2} \mathbf{I}_{3}$ \\
\hline & $\begin{array}{l}\text { Raman } \\
(150 \mathrm{~K})\end{array}$ & $\begin{array}{l}\text { Raman } \\
(150 \mathrm{~K})\end{array}$ & $\begin{array}{c}\text { Isotope } \\
\text { shift } \\
v^{12}-v^{13} \\
\end{array}$ & $\begin{array}{c}\text { Raman } \\
(10 \mathrm{~K})\end{array}$ & $\begin{array}{c}\text { Raman } \\
(15 \mathrm{~K})\end{array}$ & $\begin{array}{c}\text { Isotope } \\
\text { shift } \\
v^{12}-v^{13} \\
\end{array}$ & $\begin{array}{c}\text { Raman } \\
(20 \mathrm{~K})\end{array}$ \\
\hline$v_{2}{ }^{1}$ & 1516 & 1496 & $\sim 20$ & 1515 & 1503 & 13 & \\
\hline$v_{2}^{2}$ & - & $\sim 1484$ & - & 1500 & 1490 & 10 & 1496 \\
\hline$v_{2}^{3}$ & 1487 & 1470 & 17 & 1492 & 1479 & 14 & \\
\hline$v_{3}{ }^{1}$ & 1471 & $\sim 1415$ & $\sim 56$ & 1471 & 1423 & 48 & \\
\hline$v_{3}^{2}$ & - & - & - & $\sim 1300$ & $\sim 1250$ & $\sim 50$ & 1469 \\
\hline
\end{tabular}




\subsubsection{Fluctuation of Charge Order in Metallic Phase}

Although $v_{2}$ and $v_{27}$ of $\alpha-\mathrm{I}_{3}$ and $\alpha-\mathrm{NH}_{4} \mathrm{Hg}$ are split into three with similar split widths, the linewidth of each mode is very different. Yue et al., showed that the linewidth of metallic $\alpha-I_{3}$ is about two to three times as large as that of $\alpha-\mathrm{NH}_{4} \mathrm{Hg}$ [130]. As shown in Figure 21a, the linewidth of $v_{27}$ of $\alpha-\mathrm{I}_{3}$ is much broader than the charge-insensitive mode such as the $\mathrm{CH}_{2}$ bending mode. As the frequencies of $v_{27}$ and $v_{2}$ strongly depend upon the site charge $\left(140 \mathrm{~cm}^{-1} / e\right.$ for $v_{27}$ and $120 \mathrm{~cm}^{-1} / e$ for $\left.v_{2}\right)$ [46], such a broad linewidth should be associated with fluctuation of the site charge. Assuming that the site charge fluctuates stochastically as a Gaussian process, Yue et al., analyzed the line shape of the somewhat isolated $v_{2}{ }^{1}$ mode, and obtained a width of $\Delta v \sim 9-17 \mathrm{~cm}^{-1}$, which corresponds to the CO amplitude of $\Delta \rho \approx 0.08-0.14$. Comparing with the theoretical curve, they estimated the parameter to be $\tau \Delta v \sim 0.7-10$ and the correlation time for the fluctuation to be $\tau^{-1} \sim 1-25 \mathrm{~cm}^{-1}$. This fluctuation rate is much faster than that of $\theta-\mathrm{RbZn}$, but it is sufficiently slow for the electron dynamics. This slow fluctuation rate suggests collective motion of site charge. In contrast to the $\theta$-type BEDT-TTF salts, $\mathrm{x}$-ray diffuse scattering has not been reported. Tanaka and Yonemitsu calculated the finite temperature free energy for the horizontal, vertical, and diagonal stripes, and threefold $\mathrm{CO}$ of $\alpha-\mathrm{I}_{3}$ within the framework of the mean-field approximation, using realistic parameters and taking electron-lattice coupling into account [87]. At low temperatures, these CO states and the paramagnetic metallic state are distributed within a narrow energy range. Therefore, the free energy in the metallic phase above $T_{\mathrm{MI}}$ is approximately multiply degenerate. Yue et al., speculated that the metallic state of $\alpha-\mathrm{I}_{3}$ above $T_{\mathrm{MI}}$ involves short-range correlation of these multiple stripes to increase entropy. This situation at finite temperature resembles the case of $\theta-\mathrm{CsZn}$ in which stripe and threefold $\mathrm{CO}$ are competing. The slow fluctuation of the site charge is regarded as dynamical fluctuation of various types of $\mathrm{CO}$ domains.

Such CO fluctuation modulates the charge density at each site and broadens the linewidth of the charge-sensitive mode, when the fluctuation rate is sufficiently slow.

Figure 21. The linewidth of $v_{27}$ and $v_{2}^{3}$ of $\alpha$-(BEDT-TTF $)_{2} I_{3}$. (a) Temperature dependence of the linewidth of infrared-active $v_{27}$, which becomes very broad in the metallic phase; (b) Pressure dependence of the linewidth of Raman active $v_{2}{ }^{3}$, which decreases on increasing pressure. These figures are modified from parts of Figures 2,4 of ref. [130].
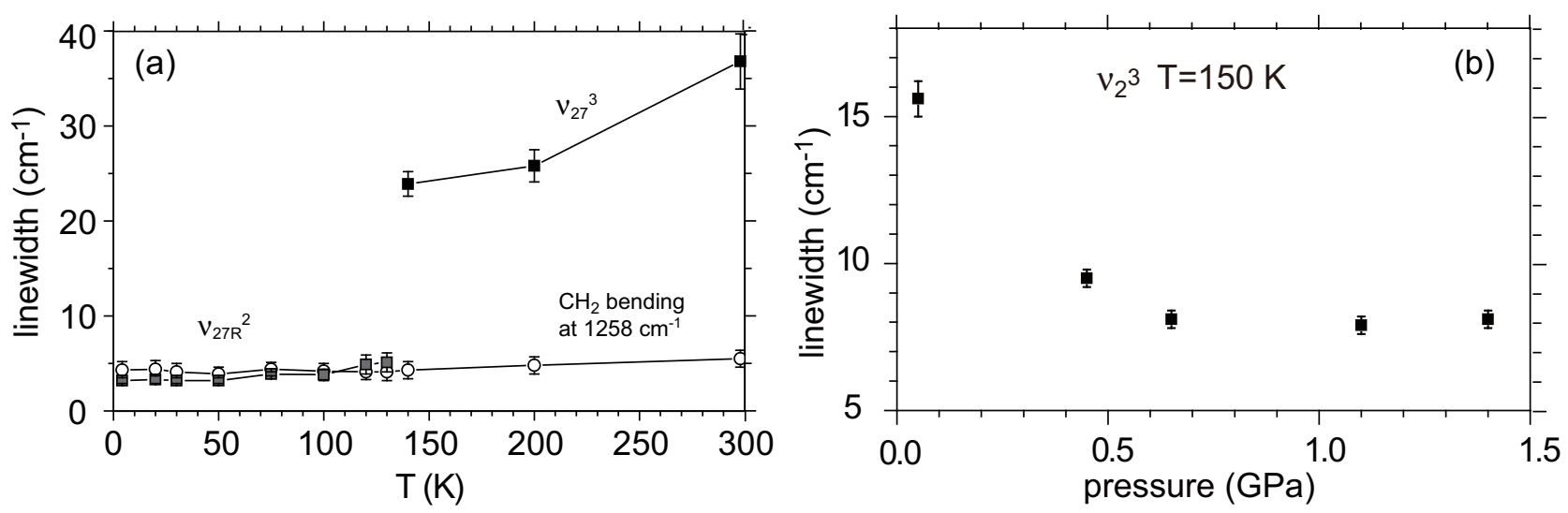
As shown in Figure 21b, the linewidth of $v_{2}{ }^{3}$ of $\alpha-\mathrm{I}_{3}$ decreases by almost half at $0.65 \mathrm{GPa}$ and it becomes comparable to the linewidth of $v_{2}^{3}$ of $\alpha-\mathrm{NH}_{4} \mathrm{Hg}$ at $100 \mathrm{~K}$. It is known that the application of hydrostatic pressure suppresses the long-range CO state [144]. This result implies that the paramagnetic metal is stabilized more than other stripe COs, and thus the energy difference between them is enlarged under hydrostatic pressure. Therefore, the fluctuation of stripe COs is suppressed under hydrostatic pressure, and the amplitude and correlation length may be reduced, leading to a higher fluctuation rate. This fast modulation narrows the linewidth of the charge-sensitive mode in addition to the small amplitude under hydrostatic pressure. The same speculation is applicable to stable metal $\alpha-\mathrm{NH}_{4} \mathrm{Hg}$. The narrow linewidth of the charge-sensitive mode of $\alpha-\mathrm{NH}_{4} \mathrm{Hg}$ is consistent with this speculation.

The existence of $\mathrm{CO}$ fluctuation in $\alpha-\mathrm{NH}_{4} \mathrm{Hg}$ is indicated by the vibronic band $v_{3}^{2}$ in the Raman spectrum (See Figure 20c, $\mathrm{c}^{\prime}$ ) as in the case of $\theta-\mathrm{CsZn}$. On the other hand, the vibronic band is not found in the Raman spectrum of the isostructural $\alpha-\mathrm{KHg}, \alpha-\mathrm{RbHg}$ and $\alpha-\mathrm{TlHg}$ salts. This results suggests that the fluctuation in $\alpha-\mathrm{NH}_{4} \mathrm{Hg}$ is largest in the group of $\alpha-\mathrm{MHg}\left(\mathrm{M}=\mathrm{NH}_{4}, \mathrm{~K}, \mathrm{Rb}, \mathrm{Tl}\right)$. Hiejima et al., observed the infrared-active $v_{27}$ modes on the side face of the crystal with the polarization perpendicular to the conducting plane [145]. In addition to the three $v_{27}$ modes, they found a weak new band growing below $200 \mathrm{~K}$ in $\alpha-\mathrm{MHg}(\mathrm{M}=\mathrm{K}, \mathrm{Rb}, \mathrm{Tl})$. They interpreted this band as the forbidden mode among the four $v_{27}$ modes, which means breaking of inversion symmetry. They speculated that fluctuation of stripe $\mathrm{CO}$ locally breaks the inversion symmetry as in $\alpha-\mathrm{I}_{3}$. If this is the case, the $\mathrm{CO}$ fluctuation in $\alpha-\mathrm{NH}_{4} \mathrm{Hg}$ is negligibly small, since the weak band is not found in $\alpha-\mathrm{NH}_{4} \mathrm{Hg}$.

To reconcile these contradictory arguments, the charge density wave (CDW) should be reconsidered. Foury-Leylekian reported an incommensurate CDW for $\alpha-\mathrm{MHg}(\mathrm{M}=\mathrm{K}, \mathrm{Rb})$ using x-ray diffraction [146]. The satellite reflections are already detected at high temperature (250-300 K). Recently, they pointed out the anion sublattice instability leading to deformation of the SCN tetrahedral coordination of $\mathrm{Hg}^{2+}$ and SCN tetragonal antiprismatic coordination of $\mathrm{K}^{+}$in $\alpha-\mathrm{KHg}$ [147]. They claimed that the Fermi-surface-nesting instability of the BEDT-TTF layer is coupled with the instability of the anion sublattice. This sublattice instability is common in $\mathrm{K}, \mathrm{Rb}$, and $\mathrm{Tl}$. As this deformation breaks inversion symmetry, the deformation of the anion sublattice brings about the non-centrosymmetric displacement of BEDT-TTF. This mechanism seems to be more reasonable to account for the breaking of the selection rule of $v_{27}$. Since this CDW is incommensurate, the $v_{27}$ modes will become broad and unresolved, if the amplitude of CDW is large enough $\Delta \rho>0.05$ [148]. Actually, the $v_{27}$ modes of $\alpha-\mathrm{NH}_{4} \mathrm{Hg}$ are more resolved than those of $\alpha-\mathrm{MHg}(\mathrm{M}=\mathrm{K}, \mathrm{Rb}, \mathrm{Tl})$ [145]. As the $v_{27}$ modes of the latter compounds are barely resolved, the CDW amplitude is considered to be $\Delta \rho<0.05$. The above discussion leads to the conclusion that the fluctuation of $\mathrm{CO}$ is indicated only in $\alpha-\mathrm{NH}_{4} \mathrm{Hg}$ which shows superconductivity.

\subsection{Non-Uniform Site-Charge Distribution in Metallic Phase}

Non-uniform site charges in $\alpha-\mathrm{I}_{3}$ have been reported using Raman spectroscopy [111] and ${ }^{13}$ C-NMR [134] experiments. Wojciechowski et al., interpreted them simply by non-uniform (anisotropic) network of transfer integrals in the unit cell using Equation (1) [111]. Yamamoto et al., conducted a systematic study of $\beta^{\prime \prime}$-type BEDT-TTF salts [149]. They qualitatively interpreted the 
site-charge distribution from non-uniform (anisotropic) intersite Coulomb interaction especially along the stacking direction. Band calculation that takes Coulomb interaction into account will incorporate the effect of both anisotropic networks on some level. Moroto et al., claimed that the non-uniform site charges are a precursor of charge order [134]. Bangura et al., attributed the splitting of $v_{2}$ in metallic $\beta^{\prime \prime}$-(BEDT-TTF $)_{4}\left[\left(\mathrm{H}_{3} \mathrm{O}\right) \mathrm{Ga}\left(\mathrm{C}_{2} \mathrm{O}_{4}\right)\right] \cdot \mathrm{C}_{6} \mathrm{H}_{5} \mathrm{NO}_{2}$ to the signature of charge order [150]. Recently, Kaiser et al., reported the splitting of $v_{27}$ in metallic $\beta^{\prime \prime}$-(BEDT-TTF) $)_{2} \mathrm{SF}_{5} \mathrm{CH}_{2} \mathrm{CF}_{2} \mathrm{SO}_{3}$, and they interpreted the growth of splitting as a fluctuation of $\mathrm{CO}$ [31]. All of these compounds involve non-equivalent sites in the unit cell: three sites in $\alpha-\mathrm{I}_{3}$ and two sites in the latter two compounds. Therefore, the splitting of the charge-sensitive mode in a metallic state is not always caused by charge order. The splitting of the charge-sensitive mode in the metallic phase should be carefully interpreted, considering the splitting width, line broadening, indication of symmetry breaking, etc. In this section, site-charges in metallic phase are more precisely discussed to compare the band calculation.

\subsubsection{Relation between Frequency and Site Charge Near $\rho=0.5$}

The relationship between the frequency of $v_{2}$ and the valence of the molecule, $\rho$, was examined by Yamamoto [46]. They assumed a linear relationship in the range of $0 \leq \rho \leq 0.8$ [151]. The $\rho$ dependence of the frequency of $v_{2}$ mode is ascribed to the $\rho$ dependent force constant $F(\rho)$. That is, the linear relationship requires $(d F / d \rho)_{\rho=0}=(d F / d \rho)_{\rho=1}$ [152]. However, the frequency of BEDT-TTF ${ }^{0.5+}$ significantly deviates from the linear relationship toward the low-frequency side, which implies the relation $\left|(d F / d \rho)_{\rho=0}\right|>\left|(d F / d \rho)_{\rho=1}\right|$. To estimate the site charge more reliably at $\rho \sim 0.5$, the empirical equation, $v_{2}(\rho)=1570-180 \rho+57 \rho^{2}(\rho \sim 0.5)$ is proposed, using $v_{2}(0)=1570 \mathrm{~cm}^{-1}$ for the calculated frequency of the flat neutral BEDT-TTF molecule, [46] $v_{2}(0.5)=1494 \mathrm{~cm}^{-1}, 1494 \mathrm{~cm}^{-1}, 1495 \mathrm{~cm}^{-1}$ respectively for $\beta^{\prime}-(\mathrm{BEDT}-\mathrm{TTF})_{2} \mathrm{ICl}_{2}$, $\kappa-(\mathrm{BEDT}-\mathrm{TTF})_{2} \mathrm{Cu}_{2}(\mathrm{CN})_{3}$, and $\kappa-(\mathrm{BEDT}-\mathrm{TTF})_{2} \mathrm{Cu}\left[\mathrm{N}(\mathrm{CN})_{2}\right] \mathrm{Cl}$ at room temperature, and $v_{2}(1)=1447 \mathrm{~cm}^{-1}$ for $(\mathrm{BEDT}-\mathrm{TTF}) \mathrm{ClO}_{4}$ and $(\mathrm{BEDT}-\mathrm{TTF}) \mathrm{AuBr}_{2} \mathrm{Cl}_{2}$ at room temperature [153]. All of the compounds with $\rho=0.5$ shows a frequency upshift by $\sim 6 \mathrm{~cm}^{-1}$ on cooling from room temperature to $10 \mathrm{~K}$ due to hardening of the crystal lattice. The frequency shift due to lattice expansion is described by the equation, $\frac{1}{\omega} \frac{d \omega}{d T}=-\gamma \beta(T)$, where $\gamma$ is the optical Gruneisen parameter and $\beta(T)$ is the coefficient of volume thermal expansion. Therefore, this temperature dependence is given by the following equation, $v_{2}(0.5, T)=\omega_{0} \exp \left\{-\gamma \int_{0}^{T} \beta(T) d T\right\}=\omega_{0} \exp \left\{-\gamma\left(\beta_{0} T+\beta_{1} T^{2} / 2\right)\right\}$, if $\beta(T)$ is approximated by the equation, $\beta(T)=\beta_{0}+\beta_{1} \mathrm{~T}$ [154]. The examples of for the $v_{2}$ modes of $\kappa-\mathrm{Cu}\left[\mathrm{N}(\mathrm{CN})_{2}\right] \mathrm{Cl}$ and $\beta^{\prime}-\mathrm{ICl}_{2}$ are shown in Figure 22. Although the function form of $\beta(\mathrm{T})$ is too crude to express actual one, the frequency shift is well reproduced. If we assume that $v_{2}(0)$ and $v_{2}(1)$ also show the same temperature dependence, the quadratic expression can be corrected as

$$
v_{2}(\rho)=1502 \exp \left(-2.8 \times 10^{-6} T-4.3 \times 10^{-8} T^{2}\right)-180 \rho+57 \rho^{2}
$$

for $T<300 \mathrm{~K}$. However, the temperature dependence of molecular vibration varies depending on the compound. For example, the $v_{2}$ mode of $\theta-\mathrm{I}_{3}$ shifts less than $3 \mathrm{~cm}^{-1}$ at $10 \mathrm{~K}$. Therefore the estimated site charge has an ambiguity of about \pm 0.03 . 
Figure 22. Temperature dependence of the frequency of $v_{2}$ of $\kappa-(\mathrm{BEDT}-\mathrm{TTF})_{2}-\mathrm{Cu}\left[\mathrm{N}(\mathrm{CN})_{2}\right] \mathrm{Cl}$ and $\beta^{\prime}-(\mathrm{BEDT}-\mathrm{TTF})_{2} \mathrm{ICl}_{2}$. The red line is the best fit equation, $\omega=A \exp \left(-B T-C T^{2} / 2\right)$, where $A=\omega_{0}, B=\gamma \beta_{0}$, and $C=\gamma \beta_{1}$.
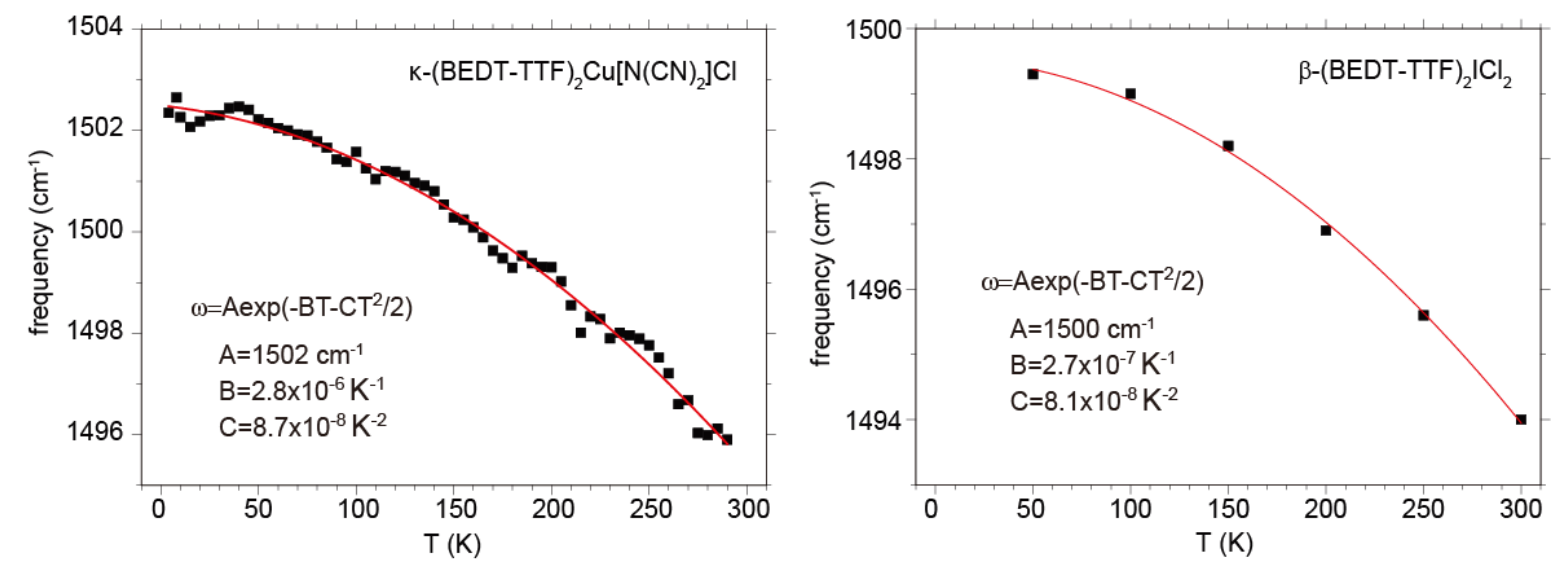

\subsubsection{Site Charge Distribution in Metallic Phase at Ambient Pressure}

The second column of Table 3 shows the site charge of $\alpha-I_{3}$ in the metallic phase estimated from the frequency of $v_{2}$ using Equation (7). According to an x-ray diffraction study, the site charge follows the order, $\rho_{\mathrm{B}}>\rho_{\mathrm{A}}=\rho_{\mathrm{A}^{\prime}}>\rho_{\mathrm{C}}$, in the metallic state [128]. $\rho_{\mathrm{A}}=\rho_{\mathrm{A}^{\prime}}$ shown in parentheses in Table 3 is calculated assuming this order. This assumption is consistent with the assignment that $v_{2}^{2}$ is missing in $\alpha-\mathrm{I}_{3}$ (See Figure 20a, $\mathrm{a}^{\prime}$ ). These hole numbers are compared with the corresponding values estimated from the infrared-active $v_{27}$ mode [130], assuming a linear relationship [155]. The site charge was calculated by Wojciechowski et al., neglecting Coulomb interaction [111]. Katayama et al., examined the effect of the anion potential, and they showed that anion potential has a small influence on the site charge [156]. Kobayashi et al., calculated the temperature dependence of the site hole number within the mean-field approximation for extended Hubbard model [157]. Ishibashi et al., made Mulliken charge analyses in their ab initio calculation of the band structure of $\alpha-\mathrm{I}_{3}$ [158]. Recently, the site hole numbers were reported by Alemany using the DFT approach considering only the valence electrons [129]. In Table 3, their results are compared with the experimental results. The mean-field calculation overestimates the site-charge difference between the sites B and C. The simplified Coulomb energy parameters may be one of the reasons for the overestimation. In the ab initio and DFT calculations, this site-charge difference is more suppressed, and the agreement with experimental values is good taking the experimental errors into account.

Table 3. Site hole numbers of metallic $\alpha$-(BEDT-TTF $)_{2} \mathrm{I}_{3}$. MF denotes mean-field calculation, and DFT denotes density functional theory.

\begin{tabular}{cccccccc}
\hline & Raman & Infrared [130] & X-ray & MF [157] & ab initio [158] & DFT [129] \\
& $\mathbf{( 1 5 0 ~ K )}$ & $\mathbf{( 1 4 0 ~ K )}$ & $\mathbf{[ 1 2 8 ]}(\mathbf{1 5 0}$ & $\mathbf{( 1 5 0 ~ K )}$ & $\mathbf{( 2 9 8 ~ K )}$ & $\mathbf{( 8 ~ K )}$ & (RT) \\
& & & $\mathbf{K})$ & & & & \\
\hline$\rho_{\mathrm{A}}=\rho_{\mathrm{A}^{\prime}}$ & $(0.52(3))$ & $\sim 0.6$ & $0.59(3)$ & 0.52 & 0.53 & 0.54 & 0.521 \\
$\rho_{\mathrm{B}}$ & $0.60(3)$ & 0.68 & $0.67(2)$ & 0.71 & 0.55 & 0.57 & 0.546 \\
$\rho_{\mathrm{C}}$ & $0.37(3)$ & 0.44 & $0.42(2)$ & 0.25 & 0.40 & 0.37 & 0.382 \\
\hline
\end{tabular}


In the same way, the site charges of metallic $\alpha-\mathrm{NH}_{4} \mathrm{Hg}$ at $10 \mathrm{~K}$ are estimated to be $0.57(3), 0.50(3)$, and 0.39(3) using the frequencies of $v_{2}$ shown in Table 2. Although the corresponding site cannot be decided by vibrational spectroscopy, Hiejima et al., assigned the three infrared-active $v_{27}$ modes with the aid of ${ }^{13} \mathrm{C}-\mathrm{NMR}$, and they interpreted the site A to be most charge-poor [145]. Apart from the result of ${ }^{13} \mathrm{C}$-NMR, the following argument also supports the above interpretation. In Section 5.2.2. we discussed the breaking of inversion symmetry in $\alpha-\mathrm{MHg}(\mathrm{M}=\mathrm{K}, \mathrm{Rb}, \mathrm{Tl})$, based on the growth of the forbidden mode. The forbidden mode is the out-of-phase mode between $v_{27 \mathrm{~A}}$ and $v_{27 \mathrm{~A}^{\prime}}$ which are coupled through dipole-dipole interaction [145]. As the dipole is induced parallel to the long axis of molecule, the frequency of the out-of-phase mode is lower than that of the in-phase mode. The out-ofphase mode grows at a slightly lower frequency of the highest frequency mode among the three $v_{27}$ modes, in-phase mode, $v_{27 \mathrm{~B}}$ and $v_{27 \mathrm{C}}$. This means that the highest frequency mode is the in-phase mode of $v_{27 \mathrm{~A}}$, that is, the A site is the most charge-poor. As the other sites cannot be decided, the assignment of $\rho_{B}$ and $\rho_{C}$ in Table 4 is interchangeable. These hole numbers are compared with the corresponding values estimated from the infrared-active $v_{27}$ mode [130], assuming a linear relationship [155]. Table 4 shows the site-charge distribution of $\alpha-\mathrm{NH}_{4} \mathrm{Hg}$ calculated by the mean-field approximation with the transfer integrals given by Mori et al. [142] and Coulomb interaction parameters, $U=0.4, V_{c}=0.17$, $V_{p}=0.05 \mathrm{eV}$ [157]. Foury-Leylekian et al., calculated the band structure of isostructural $\alpha-\mathrm{KHg}$ at $104 \mathrm{~K}$ using a numerical atomic orbital DFT approach, and presented the site charges [147]. Their values are shown in Table 4 along with the hole numbers of isostructural compounds, $\alpha-\mathrm{MHg}(\mathrm{M}=\mathrm{K}, \mathrm{Rb}, \mathrm{Rl})$. The mean-field calculation underestimates the non-uniformity among site charges, while the firstprinciples calculation enhances the non-uniformity and approaches the experimental values. However, the most charge-poor site is the B site in both theoretical calculations. This result is different from the interpretation of the experimental result of the $v_{27}$ mode (See Figure 1 of ref. [145]).

Table 4. Site hole numbers of $\alpha$-(BEDT-TTF $)_{2} \mathrm{MHg}(\mathrm{SCN})_{4}\left(\mathrm{M}=\mathrm{NH}_{4}, \mathrm{~K}, \mathrm{Rb}, \mathrm{Tl}\right) . \mathrm{MF}$ denotes mean-field calculation.

\begin{tabular}{|c|c|c|c|c|c|c|c|}
\hline & \multicolumn{3}{|c|}{$\alpha-\mathrm{NH}_{4} \mathrm{Hg}$} & \multicolumn{2}{|c|}{$\alpha-\mathrm{KHg}$} & \multirow{2}{*}{$\begin{array}{c}\alpha-\mathrm{RbHg} \\
\text { Raman } \\
(10 \mathrm{~K})\end{array}$} & \multirow{2}{*}{$\begin{array}{c}\alpha \text {-TlHg } \\
\text { Raman } \\
(10 \mathrm{~K})\end{array}$} \\
\hline & $\begin{array}{c}\text { Raman } \\
(10 \mathrm{~K})\end{array}$ & $\begin{array}{c}\text { Infrared } \\
{[130](150 \mathrm{~K})}\end{array}$ & $\begin{array}{c}\text { MF } \\
(10 \mathrm{~K})\end{array}$ & $\begin{array}{c}\text { Raman } \\
(10 \mathrm{~K})\end{array}$ & $\begin{array}{c}a b \text { initio } \\
{[147](104 \mathrm{~K})}\end{array}$ & & \\
\hline$\rho_{\mathrm{A}}=\rho_{\mathrm{A}}$ & $0.39(3)$ & 0.40 & 0.51 & $0.42(3)$ & 0.520 & $0.42(3)$ & $0.42(3)$ \\
\hline$\rho_{\mathrm{B}}$ & {$[0.50(3)]$} & {$[0.56]$} & 0.47 & {$[0.55(3)]$} & 0.424 & {$[0.55(3)]$} & {$[0.55(3)]$} \\
\hline$\rho_{\mathrm{C}}$ & {$[0.57(3)]$} & 0.62 & 0.50 & $\lfloor 0.61(3)\rfloor$ & 0.527 & $0.61(3)$ & {$[0.61(3)\rfloor$} \\
\hline
\end{tabular}

From the symmetry of the unit cell of $\theta-I_{3}$, every site is approximately equivalent. Therefore, the site charge should be $\rho=0.5$. Applying the empirical Equation (7), the site charge is calculated as $\rho=0.51(3)$ at $298 \mathrm{~K}\left(1493 \mathrm{~cm}^{-1}\right)$ and $\rho=0.54(3)\left(1496 \mathrm{~cm}^{-1}\right)$ at $20 \mathrm{~K}$. The deviation from 0.5 at $20 \mathrm{~K}$ comes from the temperature dependence of the $v_{2}$ mode that is different from that for $\kappa$-type ET salts which are used to obtain the first term of Equation (7).

\subsubsection{Site-Charge Distribution under Hydrostatic Pressure}

Tamura et al., showed that metallic $\theta-\mathrm{I}_{3}$ undergoes a first order phase transition showing a resistivity jump at around $0.5 \mathrm{GPa}$ [159]. Tajima et al., further investigated the transport properties 
under hydrostatic pressure [160]. They found that the electrical resistance under $1 \mathrm{GPa}$ behaves similar to that of $\alpha-\mathrm{I}_{3}$ at the same pressure. In addition, the Hall coefficient showed the same temperature dependence as that of $\alpha-\mathrm{I}_{3}$ at the same pressure. These two transport properties suggest a zero-gap state (ZGS) in $\theta-\mathrm{I}_{3}$ as well as in $\alpha-\mathrm{I}_{3}[120,121]$. Recently, Miyagawa et al., investigated $\theta-\mathrm{I}_{3}$ under hydrostatic pressure using ${ }^{13} \mathrm{C}-\mathrm{NMR}$ [161]. The behavior of the spin shift and spin-lattice relaxation rate is also consistent with ZGS. They suggested a structural transformation to the $\alpha$-type structure at $0.5 \mathrm{GPa}$. Figure 23a shows the pressure dependence of the Raman spectrum of $v_{2}$ and $v_{3}$ measured at $50 \mathrm{~K}$. The Raman spectrum shows a frequency upshift under hydrostatic pressure. However, no distinct change was observed, and the spectrum is different from that of $\alpha-\mathrm{I}_{3}$ under hydrostatic pressure (See Figure 23a). This result suggests that high symmetry of the unit cell of $\theta-\mathrm{I}_{3}$ is preserved at least approximately up to $3.1 \mathrm{GPa}$. If this compound is in a ZGS above $0.5 \mathrm{GPa}$, there should be a contact point at the Fermi level. However, it is concluded from Figure 23a that the band structure of $\theta-\mathrm{I}_{3}$ at 0.5 $\mathrm{GPa}$ is quite different from that of $\alpha-\mathrm{I}_{3}$. The conservation of the pseudo-symmetry provides a restriction when we speculate the crystal and band structure under hydrostatic pressure.

Figure 23. (a) Pressure dependence of the Raman spectrum of $\theta-(B E D T-T T F){ }_{2} I_{3}$ measured at $50 \mathrm{~K}$; (b) Pressure dependence of the frequency of $v_{2}$ in $\theta-(\mathrm{BEDT}-\mathrm{TTF})_{2} \mathrm{I}_{3}$.

(a)

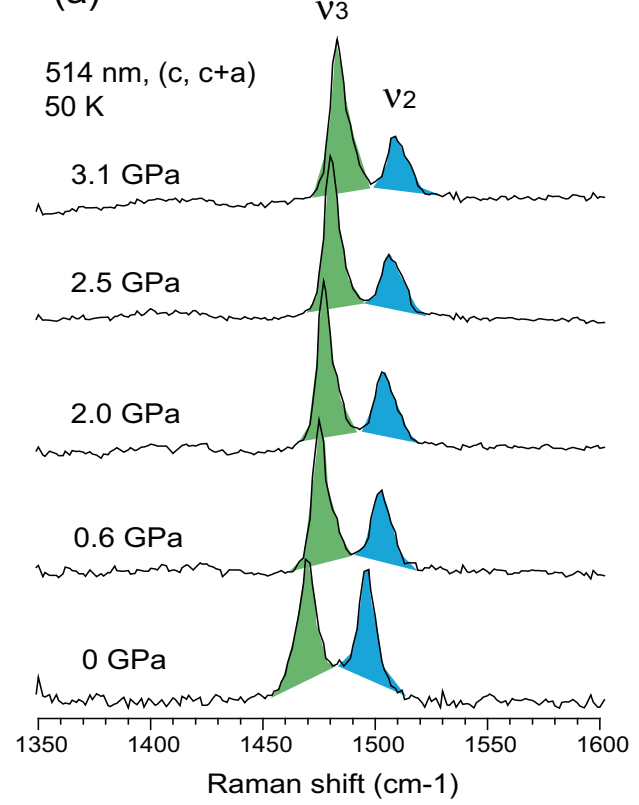

(b)

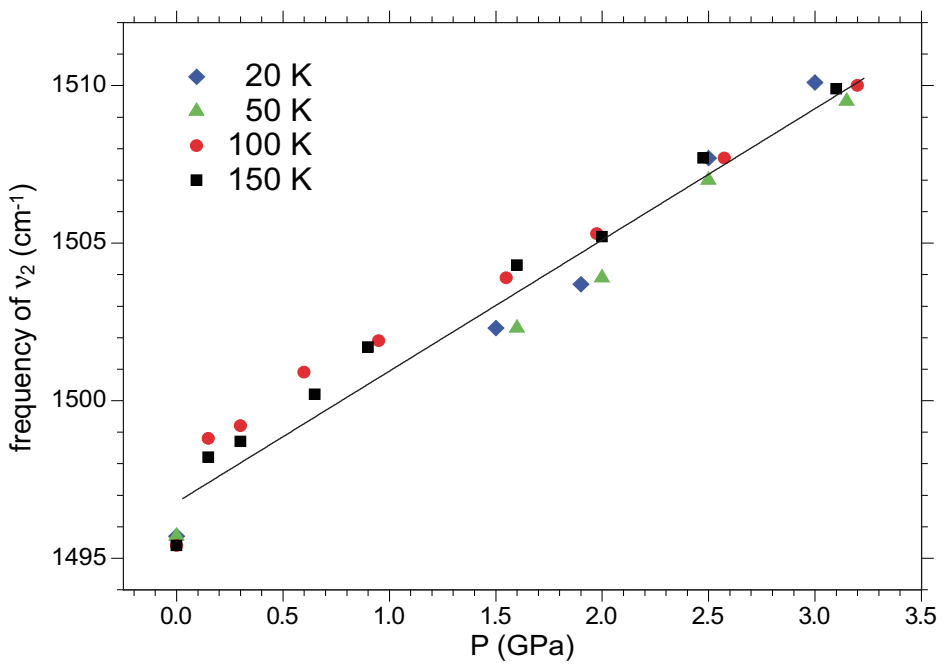

When hydrostatic pressure is applied, the molecular vibration shows a frequency upshift $(\omega+\Delta \omega)$ due to hardening of the lattice. In addition to the lattice phonon mode, the frequency shift against pressure is described by $\Delta \omega / \Delta \mathrm{P}=\omega \gamma \kappa$, where $\gamma$ and $\kappa$ are the Gruneisen constant and compressibility, respectively. Therefore, the pressure dependent frequency is given by the equation, $\omega(P)=\omega(1+\gamma \kappa P)$. It is found that $\gamma \kappa$ is approximately temperature independent within an error of $10 \%$ in the temperature range from $20 \mathrm{~K}$ to $150 \mathrm{~K}$ as shown in Figure 23b. Since the hydrostatic pressure induces no structural change in this compound, the parameter was estimated to be $\gamma \kappa \approx 3 \times 10^{-3} \mathrm{GPa}^{-1}$ from Figure $23 \mathrm{~b}$.

Figure 24 shows the pressure dependence of the Raman spectrum of $\alpha-\mathrm{I}_{3}$ at $150 \mathrm{~K}, 100 \mathrm{~K}$, and $20 \mathrm{~K}$. At $150 \mathrm{~K}$, the compound is metallic at ambient pressure. On increasing pressure, the Raman spectrum 
does not show a drastic change. At $100 \mathrm{~K}$ and $20 \mathrm{~K}$, the compound is in a CO phase at ambient pressure. In this case, remarkable spectral changes are found to occur between $0.1 \mathrm{GPa}$ and $0.45 \mathrm{GPa}$ at $100 \mathrm{~K}$ and between $1.2 \mathrm{GP}$ and $1.5 \mathrm{GPa}$ at $20 \mathrm{~K}$. These spectral changes correspond to the phase transition from the $\mathrm{CO}$ phase to the metallic phase, which is consistent with the suppression of the MI transition by hydrostatic pressure. In the metallic phase, the $v_{2}{ }^{1}$ and $v_{2}{ }^{2}$ modes do not show a parallel shift, rather they tend to merge on increasing the pressure. This trend is much more remarkable in the $\mathrm{CO}$ phase as shown in Figure 24c. Hydrostatic pressure not only hardens the lattice but also modulates the transfer integrals non-uniformly, which changes the site-charge distribution. After eliminating the lattice hardening effect assuming $\gamma \kappa \approx 3 \times 10^{-3} \mathrm{GPa}^{-1}$ for $\alpha-\mathrm{I}_{3}$, the site charge is estimated using Equation (7). The results are shown in the bottom panel of each figure. In a CO phase, the amplitude of charge order decreases on increasing pressure [162]. This trend is probably associated with the increase of transfer integrals under hydrostatic pressure.

Figure 24. Pressure dependence of the Raman spectra and site charges of $\alpha$-(BEDT-TTF $)_{2} \mathrm{I}_{3}$ analyzed at (a) $150 \mathrm{~K}$; (b) $100 \mathrm{~K}$; and (c) $20 \mathrm{~K}$. Ambiguity of the hole numbers at charge-rich site is very large, because $v_{2 R}$ is mixed with $v_{3}$ in the $\mathrm{CO}$ state. The symbols, $\boldsymbol{\Delta}$ and $\mathbf{m}$, in parentheses show the nominal site charge calculated by Equation (7). Figure 24 is modified from Figure 5 of ref. [111].

(a) $514 \mathrm{~nm}(\mathrm{~b}, \mathrm{~b}+\mathrm{a}) 150 \mathrm{~K}$
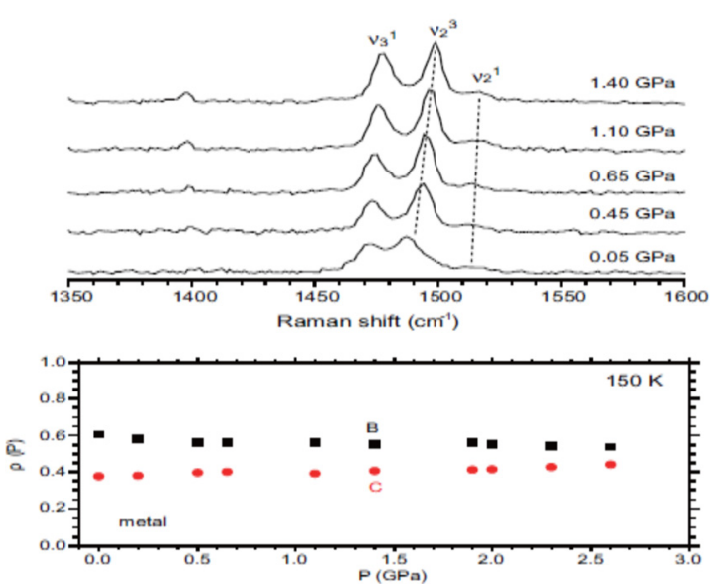

(b) $514 \mathrm{~nm}(\mathrm{~b}, \mathrm{~b}+\mathrm{a}) 100 \mathrm{~K}$
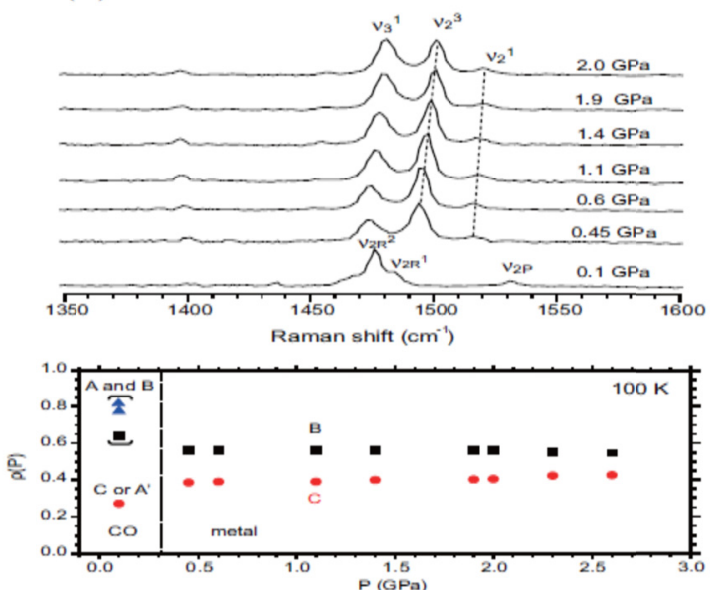
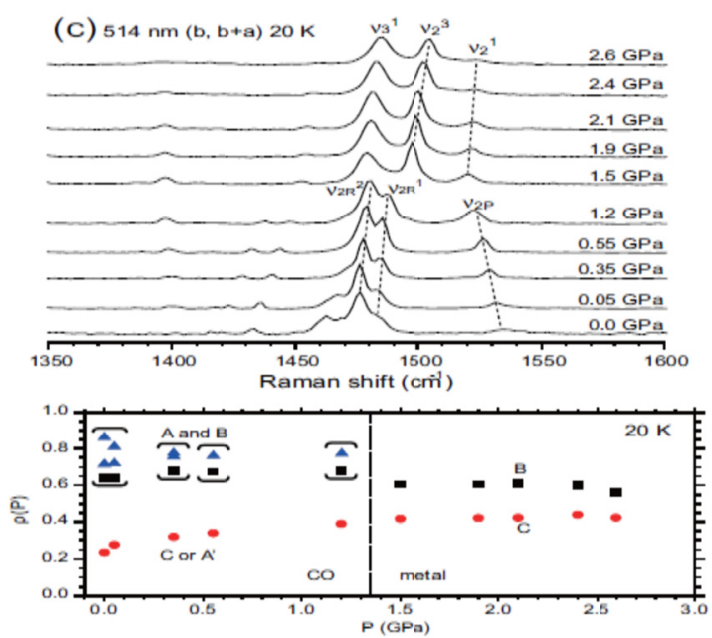
In a metallic phase, the site charges approach uniform charge distribution as in $\theta-I_{3}$ due to a non-uniform increase in transfer integrals on increasing hydrostatic pressure. This result suggests that the crystal structure of the zero-gap state of $\alpha-\mathrm{I}_{3}$ is slightly modulated so as to equalize sites B and C. However, there still remains a site-charge difference in ZGS. Alemany et al., reported the band calculation of $\alpha-\mathrm{I}_{3}$ [129] using the atomic parameters at $1.76 \mathrm{GPa}$ that is determined by Kondo et al. [163]. They used the generalized gradient approximation to DFT, and found the contact point at the Fermi level. According to their calculation, the site hole numbers changes from $\rho_{\mathrm{A}}=\rho_{\mathrm{A}^{\prime}}=0.521, \rho_{\mathrm{B}}=0.546$, $\rho_{\mathrm{C}}=0.382$ at ambient pressure to $\rho_{\mathrm{A}}=\rho_{\mathrm{A}^{\prime}}=0.576, \rho_{\mathrm{B}}=0.457, \rho_{\mathrm{C}}=0.361$ at $1.76 \mathrm{GPa}$, where the most charge-rich site is interchanged between $A=A^{\prime}$ and $B$ sites and the maximum amplitude is enlarged from $\Delta \rho_{\max }=0.164$ at ambient pressure to $\Delta \rho_{\max }=0.215$. This change is opposite to the above mentioned trend shown in Figure 24a.

\subsection{Optical Conductivity of $\alpha-(B E D T-T T F)_{2} I_{3}$}

The optical conductivity of $\alpha-\mathrm{I}_{3}$ has been published by several groups. Yakushi et al., reported optical conductivity from $720 \mathrm{~cm}^{-1}$ to $25000 \mathrm{~cm}^{-1}$ [164]. They found a drastic change in reflectivity at $\mathrm{T}_{\mathrm{MI}}$, and presented a calculation of the optical conductivity in the metallic phase based on a tight-binding approximation neglecting Coulomb interaction. The polarization (anisotropic) ratio of the optical weight was qualitatively reproduced. Zelezny et al., reported temperature dependent reflectivity in the range of $10-700 \mathrm{~cm}^{-1}$, and transmittance in the $10-33 \mathrm{~cm}^{-1}$ range [165]. They found a gap-like decrease in optical conductivity below $100 \mathrm{~cm}^{-1}$ in the $\mathrm{CO}$ phase, and they found a lattice phonon at $31.7 \mathrm{~cm}^{-1}$ in the $\mathrm{E} \| b$ spectrum at $20 \mathrm{~K}$. Dressel et al., reported optical conductivity in the range of $15-5500 \mathrm{~cm}^{-1}$ along with temperature-dependent microwave conductivity $(10,12,35,60$, and $100 \mathrm{GHz}$ ) [166]. They determined the optical gap to be $400 \mathrm{~cm}^{-1}$, and found a strong phonon at $35 \mathrm{~cm}^{-1}$ below $\mathrm{T}_{\mathrm{MI}}$. The similarity to the CDW system was pointed out from the experimental findings of non-linear transport, high dielectric permittivity, and $35 \mathrm{~cm}^{-1}$ band, although $\alpha-\mathrm{I}_{3}$ is not a CDW system. More recently, Clauss et al., reported the optical conductivity in the range of $10-10000 \mathrm{~cm}^{-1}[133,167]$. They found no Drude response above $\mathrm{T}_{\mathrm{MI}}$, and they determined the CO gap to be $600 \mathrm{~cm}^{-1}$ below $\mathrm{T}_{\mathrm{MI}}$. They estimated the effective transfer integrals comparing with theoretical calculation of the extended Hubbard model for a quarter-filled square lattice [40]. Figure 25 shows the reflectivity and optical conductivity of $\alpha-\mathrm{I}_{3}$ that is reported by Yue et al. [130]. The optical conductivity agrees well with that reported by Clauss et al. [133,167]. According to the theoretical model by Merino et al. [40], the spectral weight of the electronic excitation at $\sim 6 t$ ( $t$ is the transfer integral of square lattice) in the $\mathrm{CO}$ phase shifts to $\sim 2 t-3 t$ with Drude response in the metallic phase. The corresponding peak shift is not clear in the $\mathrm{E} \| b$ spectrum. As Clauss et al., pointed out, the spectral weight in the infrared region shifts to the gap region in the metallic phase. This behavior is typically found in the E\| $a$ spectrum. No Drude response was observed in the metallic phase. It is difficult to specify the cause of non-Drude behavior, because the metallic phase exists only at high temperature, at which thermal excitations collapse the quasi-particle band as described for the optical conductivity of $\theta-\mathrm{I}_{3}$ in Section 4.3, and/or they blur the small semimetallic Fermi surface. Incidentally, a well-defined Drude response is observed in $\alpha-\mathrm{MHg}$ $\left(\mathrm{M}=\mathrm{NH}_{4}, \mathrm{~K}, \mathrm{Rb}, \mathrm{Tl}\right)$, because these compounds are a metallic down to low temperature [29,30]. 
The collective excitation in the $\mathrm{CO}$ state has been investigated in analogy with $\mathrm{CDW}$. The experimental findings of large permittivity in the range of $50-50000 \mathrm{~Hz}$ and nonlinear conductivity of $\alpha-\mathrm{I}_{3}$ in the CO phase recalled the sliding of CDW [166]. The observation of the voltage oscillation in the $7-28 \mathrm{kHz}$ region suggests collective transport [168]. A recent permittivity experiment from $1 \mathrm{~Hz}$ to $1 \mathrm{MHz}$ indicates the existence of a large dielectric relaxation mode [133,169]. Ivek et al., interpreted this low-frequency dielectric relaxation mode to be a phason-like behavior. Dressel et al., reported an attempt to find a collective $\mathrm{CO}$ mode in the low-frequency region, and they assigned the strong 35 $\mathrm{cm}^{-1}$ band to a collective $\mathrm{CO}$ excitation [170]. However, the corresponding strong band is not indicated in ref. $[133,167]$. Yue et al., reported the transmittance of the single crystal of $\alpha-\mathrm{I}_{3}$.down to $28 \mathrm{~cm}^{-1}$ [130]. They confirmed the $31 \mathrm{~cm}^{-1}$ band which Zelezny et al., reported [165] but did not find such a strong band at $35 \mathrm{~cm}^{-1}$. More extensive experimental and theoretical studies will be necessary to establish collective $\mathrm{CO}$ excitation.

Figure 25. Temperature dependent reflectivity and optical conductivity of $\alpha$-(BEDT-TTF $)_{2} \mathrm{I}_{3}$. The optical conductivities are taken from ref. [130].
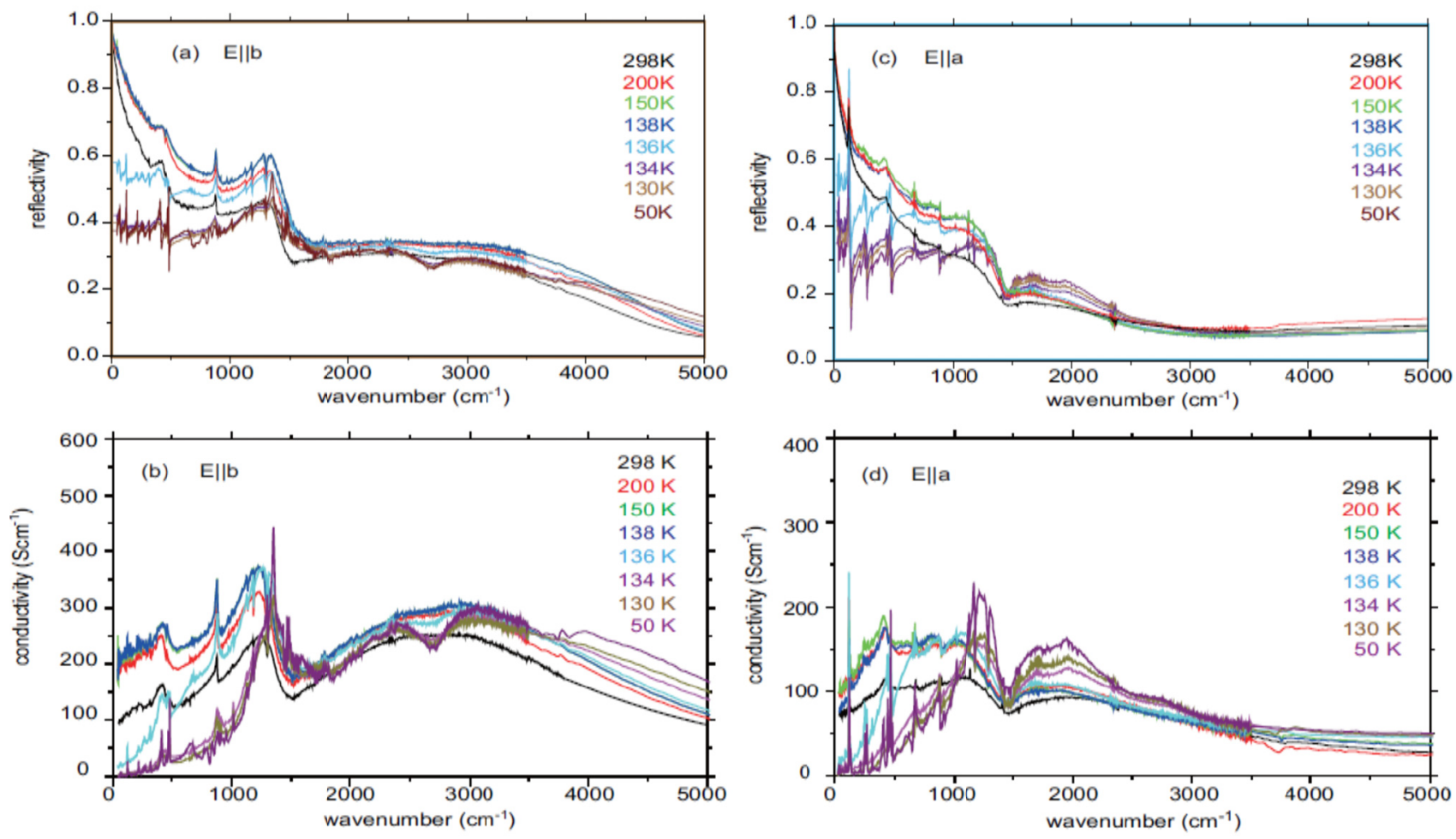

\section{5. $\alpha^{\prime}-(B E D T-T T F)_{2} \mathrm{IBr}_{2}$}

$\alpha^{\prime}$-(BEDT-TTF) $)_{2} \mathrm{IBr}_{2}$ (abbreviated as $\alpha^{\prime}-\mathrm{IBr}_{2}$ ) has a layered structure similar to $\alpha-\mathrm{I}_{3}$, but it is not isostructural with the $\alpha$-type BEDT-TTF salts [171]. Similar to $\alpha$ - $\mathrm{I}_{3}$, the unit cell contains four molecules with a herringbone arrangement. Unlike $\alpha-\mathrm{I}_{3}$, on the other hand, the unit cell contains two crystallographically independent groups $\left(\mathrm{A}, \mathrm{A}^{\prime}\right)$ and $\left(\mathrm{B}, \mathrm{B}^{\prime}\right)$, in which $\mathrm{A}$ and $\mathrm{A}^{\prime}\left(\mathrm{B}\right.$ and $\left.\mathrm{B}^{\prime}\right)$ are connected by inversion symmetry within each group. The band structure is calculated to be semimetallic, and the bandwidth of $\alpha^{\prime}-\mathrm{IBr}_{2}$ is the narrowest among the $\alpha$-type BEDT-TTF salts [171]. Reflecting the narrow bandwidth, $\alpha^{\prime}-\mathrm{IBr}_{2}$ is semiconducting ( $\rho_{\mathrm{RT}} \sim 0.3 \Omega \mathrm{cm}$ ) with an activation energy 
of $\sim 0.16 \mathrm{eV}$ at around room temperature. The electrical resistivity increases steeply by an order of magnitude at around $200 \mathrm{~K}$, and this compound moves into a high-resistivity state with the same activation energy [172]. The phase transition of $\alpha^{\prime}-\mathrm{IBr}_{2}$ was investigated by means of NMR [173] and X-ray diffraction $[171,174]$ experiments. The NMR study suggested that the thermal motion of the ethylene groups of BEDT-TTF begins to freeze gradually at around $250 \mathrm{~K}$ [173]. X-ray diffraction study confirmed this suggestion analyzing the temperature factors of the carbon atoms in one of the ethylene groups [171]. The ordering of the ethylene group occurs continuously from room temperature down to $70 \mathrm{~K}$. Except for this ordering, no distinct structural change was reported above and below $200 \mathrm{~K}$. Neither superlattice spots nor diffuse x-ray scattering has been found down to $20 \mathrm{~K}$ [171]. The magnetic susceptibility also shows no clear anomaly at around $200 \mathrm{~K}$ [172]. Inokuchi et al., reported the optical conductivity and Raman spectrum of this compound, and they suggested that the unit cell involves two non-equivalent molecules with different valence [175]. X-ray diffraction study suggested that the electron-phonon interaction is extremely weak in this system.

Yue et al., investigated the infrared and Raman spectra of $\alpha^{\prime}-\mathrm{IBr}_{2}$, and assigned the Raman spectrum with the aid of polarization dependence and ${ }^{13} \mathrm{C}$-substituted compounds [176]. Based on the assignment, they showed clear evidence that the low-temperature high-resistivity phase is a charge-ordered state. Combined with the $v_{27}$ mode, they estimated the site charge distribution to be $(0.9,0.8,0.2,0.1)$, and thus the maximum $\mathrm{CO}$ amplitude is very large, $\Delta \rho_{\max }=0.8$. Although the side band of $v_{27 \mathrm{R}^{2}}{ }^{2}$ (indicated by an arrow in Figure 26) is assigned to $\mathrm{CH}_{2}$ bending mode, it may be assigned to $v_{27 \mathrm{R}}{ }^{1}$ instead of another side band, because the low-frequency side band is observed in the deuteriumsubstituted compound. In this case, the site charge distribution and $\Delta \rho_{\max }$ are estimated to be (1.0, 0.8 , $0.2,0.1)$ and $\Delta \rho_{\max }=0.9$. Furthermore, they found that the inversion symmetry is broken in the $\mathrm{CO}$ state. If the unit cell has inversion symmetry, the $v_{2}$ mode is classified into two Raman-active (inphase) modes and two infrared-active (out-of-phase) modes. In the CO phase, they found four $v_{2}$ modes in the Raman spectrum of the ${ }^{13} \mathrm{C}$-substituted $\alpha-\mathrm{IBr}_{2}$. They took notice of the stretching vibration of $\mathrm{IBr}_{2}{ }^{-}$as a marker band for the inversion symmetry. However, the splitting of this band seems not to be decisive to judge the breaking of inversion symmetry, as the frequency of this mode is comparable with the lattice phonon. Since $\mathrm{IBr}_{2}{ }^{-}$is not located on the center of symmetry in the unit cell, the intramolecular vibration is influenced by the non-centrosymmetric crystal field. The $v_{2}$ or $v_{3}$ mode is much safer as a marker band. Taking the symmetry into account, they proposed two possible arrangements, diagonal stripes along the $a-b$ and $b$ axes, as the CO pattern. These two stripes are probably competing and generate frustration in this compound. 
Figure 26. Temperature dependences of the (a) Infrared-active $v_{27}$ mode; (b) Raman-active $v_{2}$ and $v_{3}$ modes; and (c) electrical resistivity in $\alpha^{\prime}-(\mathrm{BEDT}-\mathrm{TTF})_{2} \mathrm{IBr}_{2}$. See ref. [176] for the argument of assignment. Figure 26a,c are taken from ref. [58].
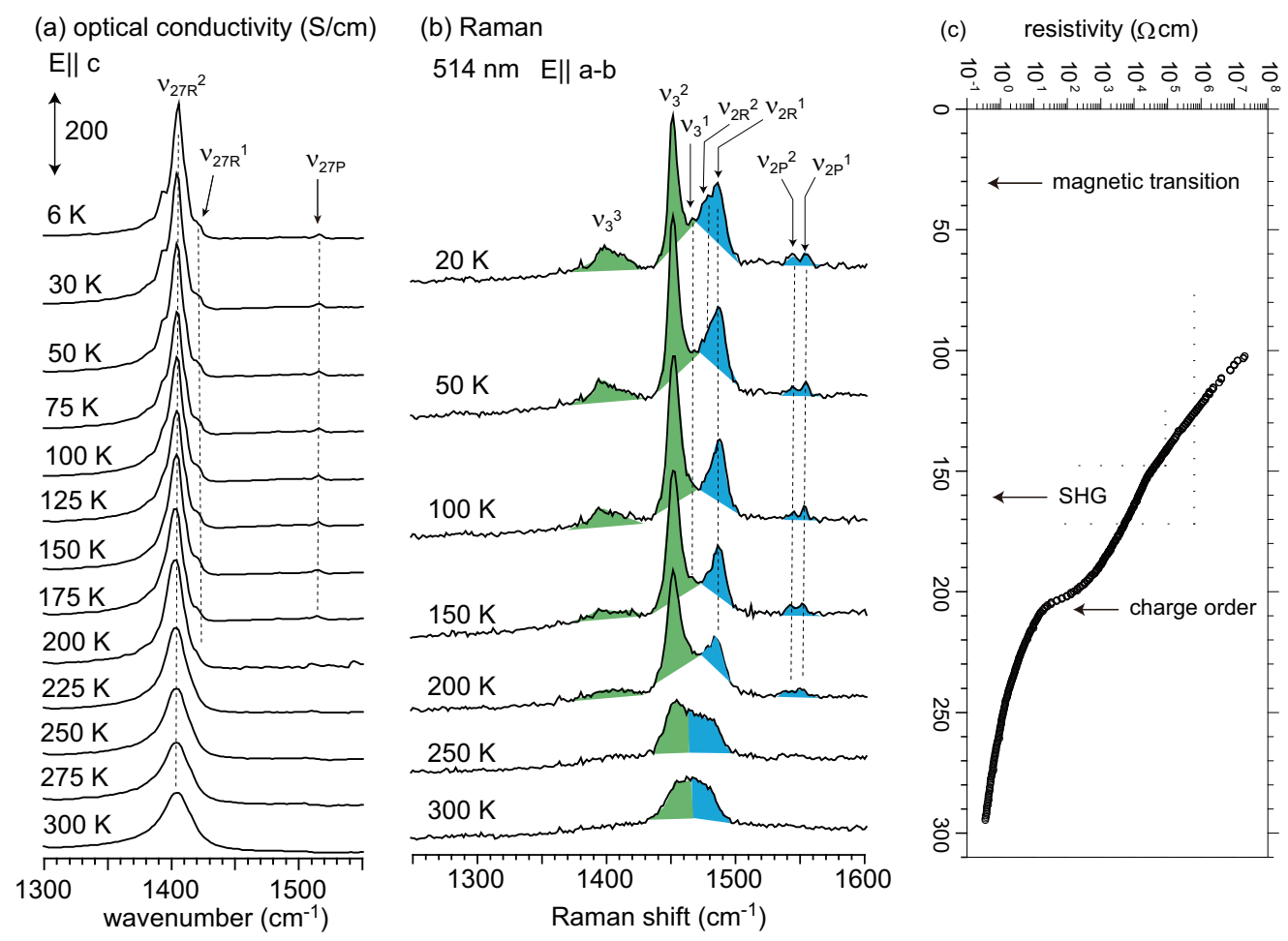

Yue et al., analyzed the resistivity and magnetic susceptibility [58]. They found that the resistivity curve is approximately expressed by a single exponential curve below $\sim 210 \mathrm{~K}$, whereas it cannot be expressed by a single exponential curve above $\sim 210 \mathrm{~K}$, where the activation energy exponentially decreases against $1 / T$. The magnetic susceptibility is expressed by an alternating Heisenberg model down to $30 \mathrm{~K}$. At around $30 \mathrm{~K}$ this compound abruptly loses susceptibility and becomes non-magnetic with hysteresis [177]. They pointed out that every crystal cracked when the crystal was cooled across this transition temperature. As the deuterium substituted $\alpha^{\prime}-\mathrm{IBr}_{2}$ does not show the $30 \mathrm{~K}$ phase transition, the interaction with $\mathrm{IBr}_{2}^{-}$may be associated with this magnetic phase transition. Yamamoto et al., found an onset of SHG at $160 \mathrm{~K}$ using an SHG microscope, and they argued for the growth of ferroelectric polarization below $160 \mathrm{~K}[136,178]$. Therefore, this compound has three phase transitions at $\sim 210 \mathrm{~K}, \sim 160 \mathrm{~K}$, and $\sim 30 \mathrm{~K}$. This behaviour is different from that of $\alpha-\mathrm{I}_{3}$, in which all of these transitions occur at the same temperature $(\sim 135 \mathrm{~K})$.

Figure 26 shows the infrared-active $v_{27}$ mode and Raman-active $v_{2}$ and $v_{3}$ modes along with the electrical resistivity [58]. The charge-sensitive $v_{27}$ and $v_{2}$ modes are already split at room temperature, and the spectral change against temperature is continuous as in $\theta-\mathrm{Cu}_{2}(\mathrm{CN})\left[\mathrm{N}(\mathrm{CN})_{2}\right]_{2}$ and $\theta_{\mathrm{m}}-\mathrm{TlZn}$. It is therefore obvious that the line shapes of the $v_{27}$ and $v_{2}$ modes reflect the case of $\tau \Delta v_{\gg} 1$ over the whole temperature range. On increasing temperature, as shown in Figure 26b, the $v_{3}$ mode is weakened toward the transition temperature at $\sim 210 \mathrm{~K}$. This spectral change suggests shortening of the coherence length $\zeta$ of the $\mathrm{CO}$ domain [58]. The optical conductivity has a clear gap below $200 \mathrm{~K}$, whereas it has finite density of states above $210 \mathrm{~K}$. The temperature dependence is continuous as for $\theta_{\mathrm{m}}-\mathrm{TlZn}$. In the limit of $\zeta=0$, the individual holes, perhaps polaron, are randomly hopping at high temperature. 
Assuming this extreme case, Yue et al., interpreted the phase transition at $210 \mathrm{~K}$ as an order-disorder transition [58]. They qualitatively explained the optical conductivity change based on the order-disorder model of the atomic limit. According to this simple model, the finite density of states above $210 \mathrm{~K}$ is generated by the disordered configurations of localized holes, the energy of which is given by the coordination number around the hole $[58,177]$. The electrical and magnetic properties and spectroscopic feature closely resemble those of $\theta-\mathrm{Cu}_{2}(\mathrm{CN})\left[\mathrm{N}(\mathrm{CN})_{2}\right]_{2}$, hence the high-temperature phase might be understood in the same way as $\theta-\mathrm{Cu}_{2}(\mathrm{CN})\left[\mathrm{N}(\mathrm{CN})_{2}\right]_{2}$, in which the short-range $\mathrm{CO}$ domain grows extensively. However, strong frustration perhaps suppresses the growth of short-range order in this compound.

This simple model cannot explain the difference in temperature between the order-disorder $(\sim 210 \mathrm{~K})$ and ferroelectric $(\sim 160 \mathrm{~K})$ transitions. In the atomic limit, the Coulomb repulsion along two diagonal stripes is nearly the same. These nearly degenerate short-range $\mathrm{CO}$ domains compete with each other and generate frustration, when the coherence length of $\mathrm{CO}$ increases. This frustration may cause the above successive ferroelectric transition. Recently, weak superlattice spots for $a \times b \times 2 c$ were found in an x-ray study conducted at BL-8A beamline of the Photon Factory Facility, KEK [179]. Based on the structural modulation, an antiferroelectric state is proposed as the intermediate phase $(160-210 \mathrm{~K})$ before the ferroelectric transition.

Yue et al., estimated the kinetic energy from the integration of optical conductivity [177], which is $0.1,0.3$, and $0.4 \mathrm{eV}$ per unit cell for $\alpha^{\prime}-\mathrm{IBr}_{2}, \alpha-\mathrm{I}_{3}$, and $\alpha-\mathrm{NH}_{4} \mathrm{Hg}$, respectively. This result indicates that the transfer integrals of $\alpha^{\prime}-\mathrm{IBr}_{2}$ are very small compared with those of $\alpha-\mathrm{I}_{3}$, and $\alpha-\mathrm{NH}_{4} \mathrm{Hg}$. Therefore, in the case of $\alpha^{\prime}-\mathrm{IBr}_{2}$, the spin degree of freedom survives after the $\mathrm{CO}$ transition due to the small spin gap $(\sim 82 \mathrm{~K})$ coming from the small exchange energy along the stripe. On the other hand, in $\alpha-\mathrm{I}_{3}$, the exchange energy is larger and the dimerization along the stripe is larger than that in $\alpha^{\prime}-\mathrm{IBr}_{2}$, and furthermore, the dimerization is enhanced below the phase transition of $\alpha-\mathrm{I}_{3}[128,171]$. These two factors result in a large spin gap $(440 \mathrm{~K})$ as reported by Sugano et al. [180]. The difference in the magnitude of spin gap is the cause of the simultaneous transition of charge order and non-magnetic state. The difference in ferroelectric transition is not clear at the moment. Possibly, the frustration plays a role in the successive transition to the ferroelectric state in $\alpha^{\prime}-\mathrm{IBr}_{2}$.

\section{Summary}

The charge-ordering phase transition arises from the competition between the kinetic energy gain and the cost of on-site and nearest-neighbor inter-site Coulomb energy. In some compounds such as $\theta$-type BEDT-TTF salts, geometrical frustration stabilizes the metallic state, and lattice distortion sometimes plays a dominant role for dissolving the frustration. Even in such a case, the transfer integral plays an essential role for the properties of the materials [78]. Transfer integrals depend considerably on the counter anions and temperature, whereas Coulomb interaction is almost insensitive to such a modification. The rough values of the kinetic energy per unit formula (=hole) are shown in Figure 27, which are estimated from the room-temperature optical conductivity [181]. The kinetic energy is a stabilization energy due to the delocalization of the valence electron over the crystal. The kinetic energy is associated with the bandwidth: the wider the bandwidth, the larger the kinetic energy. 
The kinetic energy including the correlation effect is experimentally estimated from the optical conductivity [182]. The kinetic energy $-\mathrm{E}_{\mathrm{K}}$ was calculated in the same way as reported by Yue et al. [177].

The kinetic energy of $\beta^{\prime \prime}$-(BEDT-TTF)(TCNQ) is comparable with those of $\theta_{\mathrm{m}}$-TlZn and $\theta_{\mathrm{o}}-\mathrm{TlZn}$, which undergo a metal-insulator transition while maintaining a large $C O$ amplitude $(\Delta \rho \sim 0.6)$ over the whole temperature range. In contrast to the $\theta$-type salts, this compound continuously changes the spectral feature from a large $\mathrm{CO}$ amplitude $(\Delta \rho \sim 0.6)$ to a small amplitude $(\Delta \rho \sim 0)$ on lowering the temperature. Experimentally, the split charge-sensitive modes merge into a single mode in the low-temperature region. The transport properties below $20 \mathrm{~K}$ indicate Fermi-liquid behavior. It is speculated from the optical conductivity and charge-sensitive modes that the coherent state below 20 $\mathrm{K}$ collapses above $20 \mathrm{~K}$ and continuously changes into a charge-ordered metal with a pseudogap. As the hydrostatic pressure reproduces the same spectral change for the Raman-active mode, the variation of the transfer integral due to lattice contraction has a big influence on the electronic state of this compound.

Figure 27. Kinetic energy per a formula unit (a) plotted as a function of the lattice parameter ratio. In the case of the $\theta$-type crystal, the crystal axis along the stacking direction is doubled. The inset shows the schematic molecular arrangement and crystal axes. In the compounds framed by the green rectangle, the CO amplitude is small $(\Delta \rho \sim 0.1)$ over the whole temperature range. In the compounds framed by the yellow rounded rectangle, the $\mathrm{CO}$ amplitude is large $(\Delta \rho \sim 0.6)$ over the whole temperature range. In the compound framed by the triangle, the $\mathrm{CO}$ amplitude changes at the phase transition, small amplitude $(\Delta \rho \sim 0.2)$ at high temperature and large amplitude $\left(\Delta \rho_{\max } \sim 0.6\right)$ at low temperature. In the compound framed by the inverted triangle, the amplitude changes continuously, large amplitude at high temperature $(\Delta \rho \sim 0.6)$ and small amplitude $(\Delta \rho \sim 0)$ at low temperature.

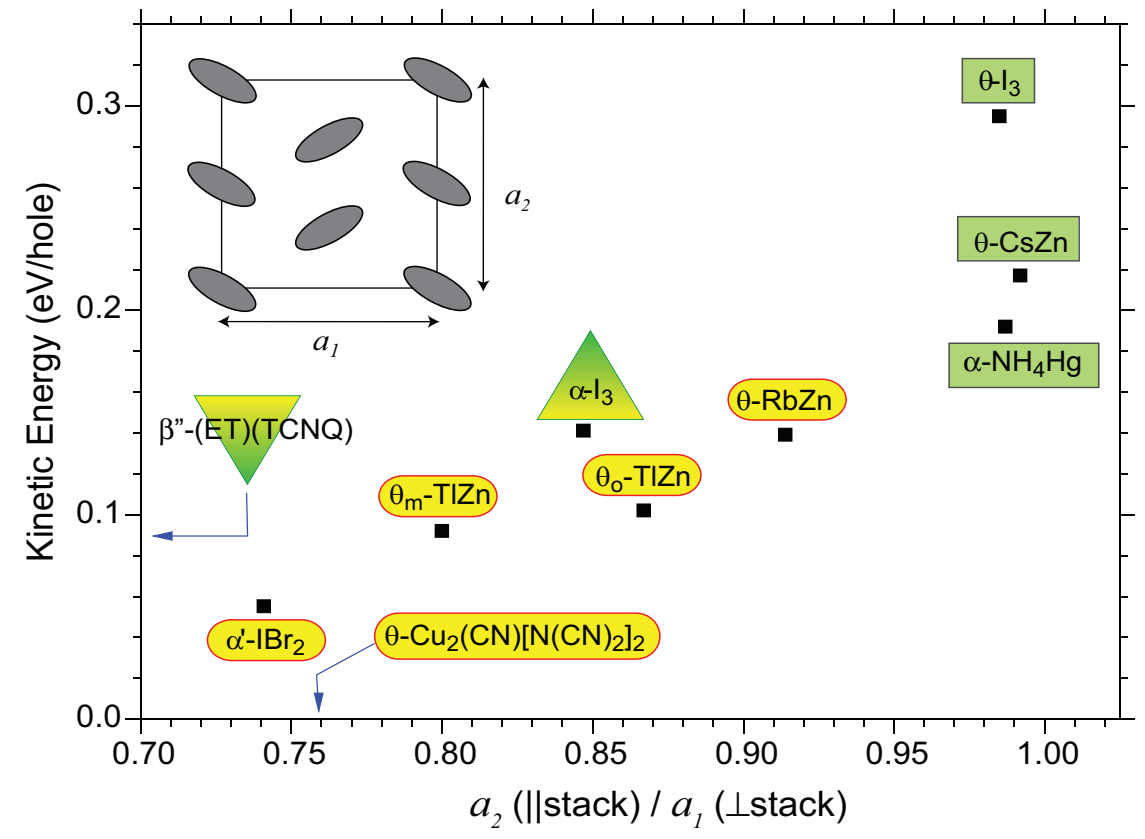


The four $\theta$-type BEDT-TTF salts, $\theta-\mathrm{RbZn}, \theta_{\mathrm{o}}-\mathrm{TlZn}, \theta_{\mathrm{m}}-\mathrm{TlZn}$, and $\theta-\mathrm{Cu}_{2}(\mathrm{CN})\left[\mathrm{N}(\mathrm{CN})_{2}\right]_{2}$, (yellow color in Figure 27) show an MI transition. However, they maintain a large CO amplitude $(\Delta \rho \sim 0.6)$ in both metallic and insulating phases. In the CO phase of the $\theta$-type BEDT-TTF salts, the CO pattern is a horizontal stripe in $\theta-\mathrm{RbZn}, \theta_{\mathrm{o}}-\mathrm{TlZn}$, and $\theta-\mathrm{Cu}_{2}(\mathrm{CN})\left[\mathrm{N}(\mathrm{CN})_{2}\right]_{2}$, as concluded by the selection rule of the $v_{3}$ modes. On the other hand, the diagonal stripe is claimed in $\theta_{\mathrm{m}}-\mathrm{TlZn}$. In contrast to the $\alpha$-type BEDT-TTF salts, the $\theta$-type compounds except $\theta_{\mathrm{m}}$-TlZn have a non-polar space group $P 2_{1} 2_{1} 2_{1}$ in the CO phase. Probably, the $\theta$-type compounds except for $\theta_{\mathrm{m}}$-TlZn do not have ferroelectric polarization in the $\mathrm{CO}$ phase in spite of the non-centrosymmetric space group. As the $\mathrm{CO}$ amplitude is large even in the metallic phase, this phase is regarded as a $\mathrm{CO}$ metal. However, the compound can be classified into two groups, $\left[\theta-\mathrm{RbZn}, \theta_{\mathrm{o}}-\mathrm{TlZn}\right]$ and $\left[\theta_{\mathrm{m}}-\mathrm{TlZn}, \theta-\mathrm{Cu}_{2}(\mathrm{CN})\left[\mathrm{N}(\mathrm{CN})_{2}\right]_{2}\right]$. The phase transition in the former is discontinuous, whereas it is continuous in the latter. The activation energy of the resistivity in the latter is significantly larger, and the precursor $v_{3}$ bands are found in the latter. In the metallic phase of the latter group, short-range stripe $\mathrm{CO}$ probably grows more extensively than in the former group.

The kinetic energy of $\theta-\mathrm{CsZn}$ and $\theta-\mathrm{I}_{3}$ is significantly larger than the others among the $\theta$-type BEDT-TTF salts (green color in Figure 27). The CO amplitude of these compounds is much smaller than in the others. The amplitude of the former is $\Delta \rho<0.08$, and $\Delta \rho<0.07$ in the latter. An indication of $\mathrm{CO}$ fluctuation is found in the Raman spectrum and optical conductivity of $\theta$-CsZn, in which the frustration between the stripe and threefold COs is dominant. $\theta-\mathrm{I}_{3}$ has a zero-energy (Drude) peak in the optical conductivity at $4 \mathrm{~K}$, while the coherent state collapses at $100 \mathrm{~K}$.

The kinetic energy of $\alpha-\mathrm{I}_{3}$ is comparable with that of $\theta-\mathrm{RbZn}$. The amplitude of CO discontinuously changes from a small amplitude (at high temperature) to a large amplitude (at low temperature) at the MI transition temperature. Below the MI transition temperature, the localized charge is arranged in a horizontal stripe involving two charge-rich sites and two charge-poor sites with an amplitude of $\Delta \rho \approx 0.4-0.6$. In the CO phase, the inversion symmetry is broken, and strong SHG is found. Observation of a macroscopic polar (ferroelectric) domain is demonstrated by SHG interferometry. A fast optical response is also reported. This unconventional ferroelectricity is called electronic ferroelectricity. In the metallic phase, the broad linewidth of the charge-sensitive mode indicates fluctuation of the $\mathrm{CO}$. This fluctuation is well suppressed by hydrostatic pressure.

The kinetic energy of $\alpha-\mathrm{NH}_{4} \mathrm{Hg}$ is significantly larger than that of $\alpha-\mathrm{I}_{3}$. The linewidth of the charge-sensitive mode is narrow in $\alpha-\mathrm{MHg}\left(\mathrm{M}=\mathrm{NH}_{4}, \mathrm{~K}, \mathrm{Rb}, \mathrm{Tl}\right)$. Among these four compounds, the inversion symmetry is locally broken in $\alpha-\mathrm{MHg}(\mathrm{M}=\mathrm{K}, \mathrm{Rb}, \mathrm{Tl})$. This phenomenon is attributed to the anion-sublattice coupled incommensurate CDW. The amplitude of the CDW is estimated to be $\Delta \rho<0.05$. On the other hand, the fluctuation of $\mathrm{CO}$ is indicated in the Raman spectrum of $\alpha-\mathrm{NH}_{4} \mathrm{Hg}$ which undergoes superconducting state at $1 \mathrm{~K}$.

$\alpha-\mathrm{I}_{3}$ in the metallic phase and $\alpha-\mathrm{NH}_{4} \mathrm{Hg}$ involve three non-equivalent sites in the unit cell. Reflecting the non-uniform site, the charge-sensitive mode split into three. The maximum site-charge difference is estimated to be $\Delta \rho_{\max } \sim 0.2$ both in metallic $\alpha-\mathrm{I}_{3}$ and $\alpha-\mathrm{NH}_{4} \mathrm{Hg}$. The most charge-poor site is the $\mathrm{C}$ site in $\alpha-\mathrm{I}_{3}$ and the A site in $\alpha-\mathrm{NH}_{4} \mathrm{Hg}$. First principle calculation agrees with the experimental results for $\alpha-\mathrm{I}_{3}$. In $\alpha-\mathrm{NH}_{4} \mathrm{Hg}$, however, there is disagreement in the most charge-poor site, between the band calculation and the interpretation of the charge-sensitive mode. The site charge distribution under hydrostatic pressure is estimated for $\alpha-\mathrm{I}_{3}$ and $\theta-\mathrm{I}_{3}$. In the metallic phase, the site charge distribution approaches a uniform charge distribution on increasing pressure. However, a non-uniform site charge 
distribution remains in the zero-gap state of $\alpha-\mathrm{I}_{3}$. The zero-gap state of $\theta-\mathrm{I}_{3}$ under hydrostatic pressure keeps uniform charge distribution unlike in $\alpha-\mathrm{I}_{3}$.

The kinetic energy of $\alpha^{\prime}-\mathrm{IBr}_{2}$ is smallest among the compounds discussed in this paper, and probably comparable with that of $\theta-\mathrm{Cu}_{2}(\mathrm{CN})\left[\mathrm{N}(\mathrm{CN})_{2}\right]_{2}$. The electrical and magnetic properties and spectroscopic feature closely resemble each other. The ground state is a non-magnetic $\mathrm{CO}$ state with large amplitude $(\Delta \rho \approx 0.6-0.8) \cdot \alpha^{\prime}-\mathrm{IBr}_{2}$ shows successive transitions at $\sim 210 \mathrm{~K}$ and $\sim 160 \mathrm{~K}$ and magnetic transition at $\sim 30 \mathrm{~K}$. The large $\mathrm{CO}$ amplitude is maintained over the whole temperature range. The ordering of charge sets in at $\sim 210 \mathrm{~K}$, the ferroelectric domain begins to grow at $\sim 160 \mathrm{~K}$, and spin singlet state is formed at $\sim 30 \mathrm{~K}$ accompanied by structural distortion.

\section{Acknowledgments}

The author deeply thanks all the collaborators for their invaluable contribution to this work. In particular, collaborations and discussions with K. Yamamoto, M. Uruichi, and T. Yamamoto of Institute for molecular science, A. Kawamoto of Hokkaido University, and H. M. Yamamoto and R. Kato of RIKEN are highly appreciated. This work was partly supported by a Grant-in-Aid for Scientific Research (Grant No. 19350074) from MEXT Japan.

\section{References and Notes}

1. Girlando, A.; Bozio, R.; Pecile, C. Infrared and Raman spectroscopic evidence of ground state charge densities at TCNQ sites in crystalline $\mathrm{Cs}_{2} \mathrm{TCNQ}_{3}$. Chem. Phys. Lett. 1974, 25, 409-412.

2. Bozio, R.; Girlando, A.; Pecile, C. Vibrational analysis of spectra of quinoid molecular ions. J. Chem. Soc. Faraday Trans. 1975, 71, 1237-1254.

3. Matsuzaki, S.; Kuwata, R.; Toyoda, K. Raman spectra of conducting TCNQ salts; estimation of the degree of charge transfer from vibrational frequencies. Solid State Commun. 1980, 33, 403-405.

4. Chappell, J.S.; Bloch, A.N.; Bryden, W.A.; Maxfield, M.; Poehler, T.O.; Cowan, D.O. Degree of charge transfer in organic conductors by infrared absorption spectroscopy. J. Am. Chem. Soc. 1980, 103, 2442-2443.

5. Kagoshima, S.; Ishiguro, T.; Anzai, H. X-ray scattering study of phonon anomalies and superstructures in TTF-TCNQ. J. Phys. Soc. Jpn. 1976, 41, 2061-2071.

6. Kondo, J.; Yamaji, K. Density correlation of classical 1-d electron gas with reference to the $4 \kappa_{\mathrm{F}}$ anomaly in TTF-TCNQ. J. Phys. Soc. Jpn. 1977, 43, 424-436.

7. Torrance, J.B. Spin waves, scattering at $4 \kappa_{\mathrm{F}}$, and spin-Peierls fluctuations in organic metal: Tetrathiafulvalene-Tetracyanoquinodimethane (TTF-TCNQ). Phys. Rev. 1978, 17, 3099-3103.

8. Hubbard, J. Generalized wigner lattices in one dimension and some applications to tetracyanoquinodimethane (TCNQ) salts. Phys. Rev. 1978, 17, 494-505.

9. Hiraki, K.; Kanoda, K. Wigner crystal type of charge ordring in an organic conductor with a quarter-filled band: (DI-DCNQI) 2 Ag. Phys. Rev. Lett. 1998, 80, 4737-4740.

10. Seo, H.; Fukuyama, H. Antiferromagntic phases of one-dimensional quarter-filled organic conductors. J. Phys. Soc. Jpn. 1997, 66, 1249-1252. 
11. Chow, D.S.; Zamborszky, F.; Alavi, B.; Tantillo, D.J.; Baur, A.; Merlic, C.A.; Brown, S.E. Charge ordering in the TMTTF family of molecular conductors. Phys. Rev. Lett. 2000, 85, 1698-1701.

12. Moldenhauer, J.; Horn, C.; Pokhondnia, K.I.; Schweitzer, D.; Heinen, I.; Keller, H.J. FT-IR absorption spectroscopy of BEDT-TTF radical salts: Charge transfer and donor-anion interaction. Synth. Metal. 1993, 60, 31-38.

13. Kino, H.; Fukuyama, H. On the phase transition of $\alpha-(\mathrm{ET})_{2} \mathrm{I}_{3}$. J. Phys. Soc. Jpn. 1995, 64, 1877-1880.

14. Takano, Y.; Hiraki, K.; Yamamoto, H.M.; Nakamura, T.; Takahashi, T. Charge disproportionation in the organic conductor, $\alpha-(B E D T-T T F)_{2} \mathrm{I}_{3}$. J. Phys. Chem. Solid 2001, 62, 393-395.

15. Miyagawa, K.; Kawamoto, A.; Kanoda, K. Charge ordering in a quasi-two-dimensional organic conductor. Phys. Rev. 2000, 62, R7679-R7682.

16. Seo, H. Charge ordering in organic ET compounds. J. Phys. Soc. Jpn. 2000, 69, 805-820.

17. McKenzie, R.H.; Merino, J.; Marston, J.B.; Sushkov, O.P. Charge ordering and antiferromagnetic exchange in layered molecular crystals of the $\theta$-type. Phys. Rev. 2001, 64, 085109:1-085109:11.

18. Kuroki, K. Theoretical aspects of charge correlations in $\theta-(\mathrm{BEDT}-\mathrm{TTF})_{2} \mathrm{X}$. Sci. Technol. Adv. Mater. 2009, 10, 024312:1-024312:11.

19. Mazumdar, S.; Clay, R.T.; Campbell, D.K. Bond-order and charge-density waves in the isotropic interacting two-dimensional quarter-filled band and the insulating state proximate to organic superconductivity. Phys. Rev. 2000, 62, 13400-13425.

20. Clay, R.T.; Mazumdar, S.; Campbell, D.K. Charge ordering in $\theta$-(BEDT-TTF) 2 X materials. $J$. Phys. Soc. Jpn. 2002, 71, 1816-1819.

21. Dayal, S.; Clay, R.T.; Li, H.; Mazumdar, S. Paired-electron crystal: Order from frustration in the quarter-filled band. Phys. Rev. 2011, 83, 245106:1-245106:12.

22. Merino, J.; McKenzie, R.H. Superconductivity mediated by charge fluctuations in layered molecular crystals. Phys. Rev. Lett. 2001, 87, 237002:1-237002:4.

23. Cano-Cortes, L.; Merino, J.; Fratini, S. Quantum critical behavior of electrons at the edge of charge order. Phys. Rev. Lett. 2010, 105, 036405:1-036405:4.

24. Takahashi, T.; Nogami, Y.; Yakushi, K. Charge ordering in organic conductors. J. Phys. Soc. Jpn. 2006, 75, 051008:1-051008:17.

25. Seo, H.; Merino, J.; Yoshioka, H.; Ogata, M. Theoretical aspects of charge ordering in molecular conductors. J. Phys. Soc. Jpn. 2006, 75, 051009:1-051009:20.

26. Dressel, M.; Drichko, N. Optical properties of two-dimensional organic conductors: Signatures of charge ordering and correlation effects. Chem. Rev. 2004, 104, 5689-5715.

27. Dressel, M. Quantum criticality in organic conductors? Fermi liquid versus non-Fermi-liquid behavior. J. Phys. Condens. Matter 2011, 23, 293201:1-293201:21.

28. Nad, F.; Monceau, P. Dielectric response of the charge ordered state in the quasi-onedimensional conductors. J. Phys. Soc. Jpn. 2006, 75, 051005:1-051005:12.

29. Dressel, M.; Drichko, N.; Schlueter, J.; Merino, J. Proximity of the layered organic conductors $\alpha$-(BEDT-TTF $)_{2} M \mathrm{Hg}(\mathrm{SCN})_{4}\left(M=\mathrm{K}, \mathrm{NH}_{4}\right)$ to a charge-ordering transition. Phys. Rev. Lett. 2003, 90, 167002:1-167002:4. 
30. Drichko, N.; Dressel, M.; Kuntscher, C.A.; Pashkin, A.; Greco, A.; Merino, J.; Schlueter, J. Electronic properties of correlated metals in the vicinity of a charge-order transition: Optical spectroscopy of $\alpha$-(BEDT-TTF $)_{2} M \mathrm{Hg}(\mathrm{SCN})_{4}\left(M=\mathrm{NH}_{4}, \mathrm{Rb}, \mathrm{Tl}\right)$. Phys. Rev. 2006, 74, 235121:1-235121:11.

31. Kaiser, S.; Dressel, M.; Sun, Y.; Greco, A.; Schlueter, J.A.; Gard, G.L.; Drichko, N. Bandwidth tuning triggers interplay of charge order and superconductivity in two-dimensional organic materials. Phys. Rev. Lett. 2010, 105, 206402:1-206402:4.

32. Tanaka, M.; Yamamoto, K.; Uruichi, M.; Yamamoto, T.; Yakushi, K.; Kimura, S.; Mori, H. Infrared and Raman study of the charge-ordered state in the vicinity of the superconducting state in the organic conductor $\beta$-(meso-DMBEDT-TTF $)_{2} \mathrm{PF}_{6}$. J. Phys. Soc. Jpn. 2008, 77, 024714:1-024714:8.

33. Morinaka, N.; Takahashi, K.; Chiba, R.; Yoshikane, F.; Niizeki, S.; Tanaka, M.; Yakushi, K.; Koeda, M.; Hedo, K.; Fujiwara, T.; et al. Superconductivity competitive with checkerboard-type charge ordering in the organic conductor $\beta$-(meso-DMBEDT-TTF $)_{2} \mathrm{PF}_{6}$. Phys. Rev. 2009, 80, 092508:1-092508:4.

34. Girlando, A.; Masino, M.; Kaiser, S.; Sun, Y.; Drichiko, N.; Dressel, M.; Mori, H. Spectroscopic characterization of charge order fluctuations in BEDT-TTF metals and superconductors. Phys. Status Solidi 2012, 249, 953-956.

35. Kobayashi, A.; Katayama, S.; Suzumura, Y. Superconductivity in charge ordered metal for quasi-two-dimensional organic conductor. J. Phys. Soc. Jpn. 2005, 74, 2897-2900.

36. Pouget, J.-P. Bond and charge ordering in low-dimensional organic conductors. Physica B 2012, 407, 1762-1770.

37. Pouget, J.-P.; Foury-Leylekian, P.; Alemany, P.; Canadell, E. Charge ordering in low dimensional organic conductors: Structural aspects. Phys. Status Solidi 2012, 249, 937-942.

38. Ouyang, J.; Yakushi, K.; Misaki, Y.; Tanaka, K. Raman spectroscopic evidence for the charge disproportionation in a quasi-two-dimensional organic conductor $\theta$-(BDT-TTP $)_{2} \mathrm{Cu}(\mathrm{NCS})_{2}$. Phys. Rev. 2001, 63, 054301:1-054301:6.

39. Yakushi, K.; Yamamoto, K.; Simonyan, M.; Ouyang, J.; Nakano, C.; Misaki, Y.; Tanaka, K. Charge-ordering and magnetic phase transitions in $\theta-(\mathrm{BDT}-\mathrm{TTP})_{2} \mathrm{Cu}(\mathrm{NCS})_{2}$. Phys. Rev. 2002, 66, 235102:1-235102:5.

40. Merino, J.; Greco, A.; McKenzie, R.H.; Calandra, M. Dynamical properties of a strongly correlated model for quarter-filled layered organic molecular crystals. Phys. Rev. 2003, 68, 245121:1-245121:15.

41. Seo, H.; Tsutsumi, K.; Ogata, M.; Merino, J. Charge fluctuation in geometrically frustrated charge ordering system. J. Phys. Soc. Jpn. 2006, 75, 114707:1-114707:8.

42. Kozlov, M.E.; Pokhondnia, K.I.; Yurchenko, A.A. The assignment of fundamental vibrations of

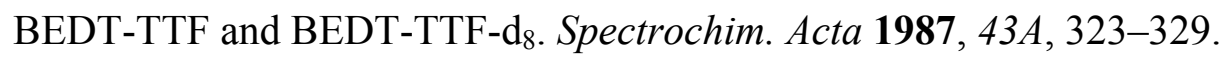

43. Kozlov, M.E.; Pokhondnia, K.I.; Yurchenko, A.A. Electron molecular vibration coupling in vibrational spectra of BEDT-TTF based radical cation salts. Spectrochim. Acta 1989, 45A, $437-444$. 
44. Eldridge, J.E.; Homes, C.C.; Williams, J.M.; Kini, A.M.; Wang, H.H. The assignment of the normal modes of the BEDT-TTF electron-donor molecule using the infrared and Raman spectra of several isotopic analogs. Spectrochim. Acta 1995, 51A, 947-960.

45. Wang, H.H.; Ferraro, J.R.; Williams, J.M.; Geiser, U.; Schlueter, J.A. Rapid Raman spectroscopic determination of the stoichiometry of microscopic quantities of BEDT-TTF-based organic conducors and superconductors. J. Chem. Soc. Chem. Commun. 1994, 1893-1894, doi:10.1039/C39940001893.

46. Yamamoto, T.; Uruichi, M.; Yamamoto, K.; Yakushi, K.; Kawamoto, A.; Taniguchi, H. Examination of the charge-sensitive vibrational modes in bis(ethylenedithio)tetrathiafulvalene. J. Phys. Chem. 2005, 109, 15226-15235.

47. Although frequency is proportional to the square root of force constant, the frequency practically linearly depends upon the force constant in a narrow frequency range, $\Delta \omega=\omega(0)-\omega(1)$.

48. Rice, M.J.; Lipari, N.O.; Strassler, S. Dimerized organic linear-chain conductors and the unambiguous experimental determination of electron-molecular-vibration coupling constants. Phys. Rev. Lett. 1977, 21, 1357-1362.

49. Rice, M.J. Towards the experimental determiation of the fundamental microscopic parameters of organic ion-radical compounds. Solid State Commun. 1979, 31, 93-98.

50. Girlando, A.; Bozio, R.; Pecile, C.; Torrance, J.B. Discovery of vibronic effects in the Raman spectra of mixed-stack charge-transfer crystals. Phys. Rev. 1982, 26, 2306-2309.

51. Painelli, A.; Girlando, A. Electron-molecular vibration (e-mv) coupling in charge-transfer compounds and its consequences on the optical spectra: A theoretical framework. J. Chem. Phys. 1986, 84, 5655-5671.

52. Yamamoto, K.; Yakushi, K. Electron-molecular vibration coupling effect on the Raman spectrum of organic charge transfer salts. J. Phys. (France) 2004, 114, 153-155.

53. Visentini, G.; Masino, M.; Bellitto, C.; Girlando, A. Experimental determination of BEDT-TTF electron-molecular vibration coupling constants through optical microreflectance. Phys. Rev. 1998, 58, 9460-9467.

54. Yartsev, V.M.; Graja, A. Electron-intramolecular vibration coupling in charge-transfer salts studied by infrared spectroscopy. Int. J. Mod. Phys. 1998, 12, 1643-1672.

55. Faltermeier, D.; Barz, J.; Dumm, M.; Dressel, M.; Drichko, N.; Petrov, B.; Semkin, V.; Vlasova, R.; Meziere, C.; Batil, P. Bandwidth-controlled Mott transition in $\kappa-(\mathrm{BEDT}-\mathrm{TTF})_{2} \mathrm{Cu}\left[\mathrm{N}(\mathrm{CN})_{2}\right]-\mathrm{Br}_{x} \mathrm{Cl}_{1-x}$ : Optical studies of localized charge excitations. Phys. Rev. 2007, 76, 165113:1-165113:12.

56. Girlando, A. Charge sensitive vibrations and electron-molecular vibration coupling in bis(ethylenethio)-thetrathiafulvalene (BEDT-TTF). J. Phys. Chem. 2011, 115, 19371-19378.

57. Yamamoto, T.; Yakushi, K.; Shimizu, Y.; Saito, G. Infrared and Raman study of the charge-ordered state of $\theta-(\mathrm{ET})_{2} \mathrm{Cu}(\mathrm{CN})\left[\mathrm{N}(\mathrm{CN})_{2}\right]_{2}$. J. Phys. Soc. Jpn. 2004, 73, 2326-2332.

58. Yue, Y.; Nakano, C.; Yamamoto, K.; Uruichi, M.; Wojciechowski, R.; Inokuchi, M.; Yakushi, K.; Kawamoto, A. Charge order-disorder phase transition in $\alpha^{\prime}$-(Bis(ethylenedithio) tetrathiafulvalene $)_{2} \mathrm{IBr}_{2}\left[\alpha^{\prime}-(\mathrm{BEDT}-\mathrm{TTF})_{2} \mathrm{IBr}_{2}\right]$. J. Phys. Soc. Jpn. 2009, 78, 044701:1-044701:10.

59. Kubo, R. A stochastic theory of line shape. Adv. Chem. Phys. 1969, 15, 101-127.

60. Sue, J.; Yan, Y.J.; Mukamel, S. Raman excitation profiles of polyatomic molecules in condensed phases. A stochastic theory. J. Chem. Phys. 1986, 85, 462-474. 
61. Uruichi, M.; Yakushi, K.; Yamamoto, H.M.; Kato, R. Infrared and Raman studies of the charge-ordering phase transition at $\sim 170 \mathrm{~K}$ in the quarter-filled organic conductor, $\beta^{\prime \prime}$-(ET) (TCNQ). J. Phys. Soc. Jpn. 2006, 75, 074720:1-074720:10.

62. Yamamoto, H.M.; Hagiwara, M.; Kato, R. New phase of (BEDT-TTF)(TCNQ). Synth. Metal. 2003, 133, 449-451.

63. Nogami, Y. Okayama University, Private communication, 2009.

64. Yamamoto, H.M.; Tajima, N.; Hagiwara, M.; Kato, R.; Yamaura, J.I. Strange electric magnetic behaviour of new (BEDT-TTF)(TCNQ). Synth. Metal. 2003, 135, 623-624.

65. Yasuzuka, S.; Terakura, C.; Terashima, T.; Yakabe, T.; Terai, Y.; Yamamoto, H.M.; Kato, R.; Uji, S. Fermi surface and resistance anomalies in ET-TCNQ. Synth. Metal. 2003, 135, 647-648.

66. Yasuzuka, S.; Graf, D.; Choi, E.S.; Brooks, J.S.; Terashima, T.; Konoike, T.; Enomoto, K.; Nishimura, M.; Yamamoto, H.M.; Kato, R.; et al. Pressure-induced Fermi surface change in quasi-one-dimensional conductor $\beta "$ "-(ET)(TCNQ). J. Phys. IV Fr. 2004, 114, 157-158.

67. Yasuzuka, S.; Uji, S.; Enomoto, K.; Konoike, T.; Nishimura, M.; Terashima, T.; Graf, D.; Choi, E.S.; Brooks, J.S.; Yamamoto, H.M.; et al. Pressure effect on fermi surface in $\beta^{\prime \prime}-(\mathrm{ET})(\mathrm{TCNQ})$. Synth. Metal. 2005, 152, 437-440.

68. Kimata, M.; Ohta, H.; Koyama, K.; Oshima, Y.; Motokawa, M.; Yamamoto, H.M.; Kato, R. Development of the high field magneto-optical measurement system with a rotational cavity for the study of organic conductors. Int. J. Mod. Phys. 2004, 18, 3803-3806.

69. Kimata, M.; Oshima, Y.; Ohta, H.; Koyama, K.; Motokawa, M.; Yamamoto, H.M.; Kato, R. Magnetooptical measurements of $\beta^{\prime \prime}$-(BEDT-TTF)(TCNQ). Physica B 2004, 346, 382-386.

70. Yakushi, K.; Uruichi, M.; Yamamoto, H.M.; Kato, R. Dynamical fluctuation of the site-charge density in metallic $\beta "$ "-(BEDT-TTF)(TCNQ). J. Phys. IV Fr. 2004, 114, 149-151.

71. Drichko, N.; Kaiser, S.; Sun, Y.; Clauss, C.; Dressel, M.; Mori, H.; Schlueter, J.; Zhyliaeva, E.I.; Torunova, S.A.; Lyubovskaya, R.N. Evidence for charge order in organic superconductors obtained by vibrational spectroscopy. Physica B 2009, 404, 490-493.

72. Yamamoto, K.; Yakushi, K.; Miyagawa, K.; Kanota, K.; Kawamoto, A. Charge ordering in $\theta$-(BEDT-TTF $)_{2} \operatorname{RbZn}(\mathrm{SCN})_{4}$ studied by vibrational spectroscopy. Phys. Rev. 2002, 65, 085110:1-085110:8.

73. There is another incoherent optical excitation at $\sim U$ with very weak intensity, which corresponds to the optical transition from the lower Hubbard to the upper Hubbard band.

74. Merino, J. Nonlocal Coulomb Correlation in metals close to a charge order insulator transition. Phys. Rev. Lett. 2007, 99, 036404:1-036404:4.

75. Kondo, R.; Higa, M.; Kagoshima, S.; Hoshino, H.; Mori, T.; Mori, H. Electrical and structural properties of $\theta$-type BEDT-TTF organic conductors under uniaxial strain. J. Phys. Soc. Jpn. 2006, 75, 044716:1-044716:7.

76. Kuroki, K. The origin of the charge ordering and its relevance to superconductivity in $\theta$-(BEDT-TTF $)_{2} \mathrm{X}$ : The effect of the fermi surface nesting and the distant electron-electron interactions. J. Phys. Soc. Jpn. 2006, 75, 114716:1-114716:14.

77. Hotta, C. Classification of quasi-two dimensional organic conductors based on a new minimal model. J. Phys. Soc. Jpn. 2003, 72, 840-853. 
78. Watanabe, H.; Ogata, M. Novel charge order and superconductivity in two-dimensional frustrated lattice at quarter filling. J. Phys. Soc. Jpn. 2006, 75, 063702:1-063702:4.

79. Mori, H.; Tanaka, S.; Mori, T. Systematic study of the electronic state of $\theta$-type BEDT-TTF organic conductors by changing electronic correlation. Phys. Rev. 1998, 57, 12023-12029.

80. Kobayashi, H.; Kato, R.; Kobayashi, A.; Nishio, Y.; Kajita, K.; Sasaki, W. New molecular superconductor (BEDT-TTF $)_{2}\left(\mathrm{I}_{3}\right)_{x}\left(\mathrm{AuI}_{2}\right)_{1-x}(x<0.02)$. Chem. Lett. 1986, 789-792.

81. Mori, H.; Tanaka, S.; Mori, T.; Kobayashi, A.; Kobayashi, H. Crystal structure and physical properties of $\mathrm{M}=\mathrm{Rb}$ and $\mathrm{Tl}$ salts of (BEDT-TTF $)_{2} \mathrm{MM}^{\prime}(\mathrm{SCN})_{4},\left[\mathrm{M}^{\prime}=\mathrm{Co}, \mathrm{Zn}\right]$. Bull. Chem. Soc. Jpn. 1998, 71, 797-806.

82. Komatsu, T.; Sato, H.; Nakamura, T.; Matsukawa, N.; Yamochi, H.; Saito, G.; Kusunoki, M.; Sakagushi, K.; Kagoshima, S. The electrical and magnetic properties of a novel two-dimensional antiferromagnet based on BEDT-TTF, $\theta-(\mathrm{BEDT}-\mathrm{TTF})_{2} \mathrm{Cu}(\mathrm{CN})\left[\mathrm{N}(\mathrm{CN})_{2}\right]_{2}$. Bull Chem. Soc. Jpn. 1995, 68, 2233-2244.

83. The doublet of $v_{2 \mathrm{P}}$ is interpreted as the Fermi resonance [72].

84. Watanabe, M.; Noda, Y.; Nogami, Y.; Mori, H. Transfer integrals and spatial pattern of charge ordering in $\theta$-(BEDT-TTF $)_{2} \mathrm{RbZn}(\mathrm{SCN})_{4}$ at 90 K. J. Phys. Soc. Jpn. 2004, 73, 116-122.

85. Suzuki, K.; Yamamoto, K.; Yakushi, K. Charge-ordering transition in orthorhombic and monoclinic single crystals of $\theta$-(BEDT-TTF $)_{2} \mathrm{TlZn}(\mathrm{SCN})_{4}$ studied by vibrational spectroscopy. Phys. Rev. 2004, 69, 085114:1-085114:11.

86. Tajima, H.; Kyoden, S.; Mori, H.; Tanaka, S. Estimation of charge-ordering patterns in $\theta-\mathrm{ET}_{2} M M^{\prime}(\mathrm{SCN})_{4}\left(M M^{\prime}=\mathrm{RbCo}, \mathrm{RbZn}, \mathrm{CsZn}\right)$ by reflection spectroscopy. Phys. Rev. 2000, 62, 9378-9385.

87. Tanaka, Y.; Yonemitsu, K. Effects of electron-lattice coupling on charge order in $\theta-(\mathrm{ET})_{2} \mathrm{X}$. J. Phys. Soc. Jpn. 2008, 77, 034708:1-034708:9.

88. Chiba, R.; Hiraki, K.; Takahashi, T.; Yamamoto, H.M.; Nakamura, T. Extremely slow charge fluctuations in the metallic state of the two-dimensional molecular conductor $\theta$-(BEDT-TTF $)_{2}$ RbZn(SCN)4. Phys. Rev. Lett. 2004, 93, 216405:1-216405:4.

89. Nakamura, T.; Miyagawa, W.; Kinami, R.; Konishi, Y.; Takahashi, T. Low-temperature electronic states in $\theta$-(BEDT-TTF $)_{2} \mathrm{RbZn}(\mathrm{SCN})_{4}$ : Competition of different ground states. Synth. Metal. 1999, 103, 1898-1899.

90. Kanoda, K.; Ohnou, K.; Kodama, M.; Miyagawa, K.; Itou, T.; Hiraki, K. Charge ordering and frustration in organic conductors. J. Phys. IV Fr.2005, 131, 21-26.

91. Watanabe, M.; Noda, Y.; Nogami, Y.; Mori, H. Investigation of X-ray diffuse scattering in $\theta$-(BEDT-TTF) ${ }_{2} \mathrm{RbM}^{\prime}(\mathrm{SCN})_{4}$. Synth. Metal. 2003, 135, 665-666.

92. Nogami, Y.; Hanasaki, N.; Watanabe, M.; Yamamoto, K.; Ito, T.; Ikeda, N.; Ohsumi, H.; Tokawa, H.; Noda, Y.; Terasaki, I.; et al. Charge order competition leading to nonlinearity in organic thyristor family. J. Phys. Soc. Jpn. 2010, 79, doi:10.1143/JPSJ.79.044606.

93. A weak broad $v_{3}$ band is probably involved in the low-frequency region of the broad $v_{2}$ band. The assignment of the broad $v_{2}$ band with the aid of isotope shift is given in ref. [94].

94. Suzuki, K.; Yamamoto, K.; Yakushi, K.; Kawamoto, A. Infrared and Raman studies of $\theta$-(BEDT-TTF $)_{2} \mathrm{CsZn}(\mathrm{SCN})_{4}$ : Comparison with the frozen state of $\theta$-(BEDT-TTF $)_{2} \mathrm{RbZn}(\mathrm{SCN})_{4}$. J. Phys. Soc. Jpn. 2005, 74, 2631-2639. 
95. Wang, N.L.; Mori, H.; Tanaka, S.; Dong, J.; Clayman, B.P. Far infrared study of insulator-metal transition in $\theta$-(BEDT-TTF $)_{2} \operatorname{RbZn}(\mathrm{SCN})_{4}$. J. Phys. Condens. Matter 2001, 13, 5463-5470.

96. Nishimoto, S.; Shingai, M.; Ohta, Y. Coexistence of distinct charge fluctuations in $\theta$-(BEDTTTF $)_{2}$ X. Phys. Rev. 2008, 78, 035113:1-035113:9.

97. The reason why the $v_{2}$ mode of metallic $I_{3}$ salt is significantly deviated from the center of $v_{2 P}$ and $v_{2 R}$ is ascribed to the interaction between $v_{2 R}$ and the neighbor $v_{3 A} \cdot[46]$.

98. Mori, T. Non-Stripe charge order in the $\theta$-phase organic conductors. J. Phys. Soc. Jpn. 2003, 72, $1469-1475$.

99. Mori, T. Estimation of off-site coulomb integrals and phase diagrams of charge ordered states in the $\theta$-phase organic conductor. Bull. Chem. Soc. Jpn. 2000, 73, 2243-2253.

100. Watanabe, M.; Nogami, Y.; Oshima, K.; Mori, H.; Tanaka, S. Novel pressure-induced $2 \kappa_{\mathrm{F}}$ CDW state in organic low-dimensional compound $\theta-(\mathrm{BEDT}-\mathrm{TTF})_{2} \mathrm{CsCo}(\mathrm{SCN})_{4}$. J. Phys. Soc. Jpn. 1999, 68, 2654-2663.

101. Watanabe, M.; Yamamoto, K.; Ito, T.; Nakashima, Y.; Tanabe, M.; Hanasaki, N.; Ikeda, N.; Nogami, Y.; Ohsumi, H.; Toyokawa, H.; et al. Non-thermal evidence for current-induced melting of charge order in $\theta$-(BEDT-TTF $)_{2}$ CsZn(SCN) 4 . J. Phys. Soc. Jpn. 2008, 77, 065004:1-065004:2.

102. Inagaki, K.; Terasaki, I.; Mori, H.; Mori, T. Large dielectric constant and giant nonlinear conduction in organic conductor $\theta-(\mathrm{BEDT}-\mathrm{TTF})_{2} \mathrm{CsZn}(\mathrm{SCN})_{4}$. J. Phys. Soc. Jpn. 2004, 73, 3364-3369.

103. Sawano, F.; Terasaki, I.; Mori, H.; Mori, T.; Watanabe, M.; Ikeda, N.; Nogami, Y.; Noda, Y. An organic thyristor. Nature 2005, 437, 522-524.

104. Chiba, R.; Hiraki, K.; Takahashi, T.; Yamamoto, H.M.; Nakamura, T. Charge disproportionation and dynamics in $\theta$-(BEDT-TTF $)_{2} \operatorname{CsZn}(\mathrm{SCN})_{4}$. Phys. Rev. 2008, 77, 115113:1-115113:10.

105. Nad, F.; Monceau, P.; Yamamoto, H.M. A possible glass-like state in $\theta$-(BEDT-TTF) $)_{2}$ $\mathrm{CsZn}(\mathrm{SCN})_{4}$ at low temperature. J. Phys. Condens. Matter 2008, 20, 485211:1-485211:6.

106. Udagawa, M.; Motome, Y. Charge ordering and coexistence of charge fluctuations in quasi-two-dimensional organic conductors $\theta$-(BEDT-TTF $)_{2}$ X. Phys. Rev. Lett. 2007, 98, 206405:1-206405:4.

107. Kajita, K.; Nishio, Y.; Moriyama, S.; Sasaki, W.; Kato, R.; Kobayashi, H.; Kobayashi, A. New organic suprconductors $\kappa-$ and $\theta$-(BEDT-TTF $)_{2} \mathrm{I}_{3}$; Transport property. Solid State Commun. 1987, $64,1279-1284$.

108. Salameh, B.; Nothardt, A.; Balthes, E.; Schmidt, W.; Schweitzer, D.; Strempfer, J.; Hinrichsen, B.; Jansen, M.; Maude, D.K. Electronic properties of the organic metals $\theta$-(BEDT-TTF) $)_{2} \mathrm{I}_{3}$ and $\theta_{\mathrm{T}}$-(BEDT-TTF) ${ }_{2} \mathrm{I}_{3}$. Phys. Rev. 2007, 75, 054509:1-054509:13.

109. The symmetry of $3 a \times 3 c$ superlattice has not been clarified.

110. Wang, N.L.; Feng, T.; Chen, Z.J.; Mori, H. Infrared properties of $\theta$-type ET charge-transfer salts: $\theta-(\mathrm{ET})_{2} \mathrm{RbZn}(\mathrm{SCN})_{4}$ vs. $\theta-(\mathrm{ET})_{2} \mathrm{CsZn}(\mathrm{SCN})_{4}$. Synth. Metal. 2003, 135, 701-702.

111. Wojciechowski, R.; Yamamoto, K.; Yakushi, K.; Inokuchi, M.; Kawamoto, A. High-pressure Raman study of the charge ordering in $\alpha$-(BEDT-TTF $)_{2} \mathrm{I}_{3}$. Phys. Rev. 2003, 67, 224105:1224105:11.

112. Takenaka, K.; Tamura, M.; Tajima, N. Collapse of coherence quasiparticle states in $\theta$-(bedtttf) $)_{2} \mathrm{i}_{3}$ observed by optical spectroscopy. Phys. Rev. Lett. 2005, 95, 227801:1-227801:4. 
113. Kobayashi, A.; Kato, R.; Kobayashi, H.; Moriyama, S.; Nishio, Y.; Kajita, K.; Sasaki, W. Anion arrangement in a new molecular superconductor, $\theta$-(BEDT-TTF $)_{2}\left(\mathrm{I}_{3}\right)_{1-x}\left(\mathrm{AuI}_{2}\right)_{x},(x<0.02)$. Chem. Lett. 1986, 2017-2020.

114. Tamura, M.; Yakushi, K.; Kuroda, H. Temperature dependence of the polarized reflectance spectra of the $\theta$-Type of bis(ethylenedithio)tetrathiafulvalenium) triiodide $\theta$-(BEDT-TTF $)_{2} \mathrm{I}_{3}$ : Estimation of band parameters. J. Phys. Soc. Jpn. 1988, 57, 3239-3247.

115. ACS. Molecular Conductors: Thematic Issue (Special issue). ACS Publication: Washington, DC, USA, 2004; pp. 4887-5781.

116. Mori, T. Structural genealogy of BEDT-TTF-based organic conductors I. Parallel molecules: $\beta$ and $\beta^{\prime \prime}$ phases. Bull. Chem. Soc. Jpn. 1998, 71, 2509-2526.

117. Mori, T. Structural genealogy of BEDT-TTF-based organic conductors II. Inclined molecules: $\theta$ and $\alpha$-, and $\kappa$-phases. Bull. Chem. Soc. Jpn. 1999, 72, 179-197.

118. Mori, T. Structural genealogy of BEDT-TTF-based organic conductors III. Twisted molecules: $\delta$ - and $\alpha^{\prime}$-phases. Bull. Chem. Soc. Jpn. 1998, 72, 2011-2027.

119. Takano, Y.; Hiraki, K.; Yamamoto, H.M.; Nakamura, T.; Takahashi, T. Charge ordering in $\alpha$-(BEDT-TTF $)_{2} \mathrm{I}_{3}$. Synth. Metal. 2001, 120, 1081-1082.

120. Tajima, N.; Ebina-Tajima, A.; Tamura, M.; Nishio, Y.; Kajita, K. Effects of uniaxial strain on transport properties of organic conductor $\alpha-(\mathrm{BEDT}-\mathrm{TTF})_{2} \mathrm{I}_{3}$ and discovery of superconductivity. J. Phys. Soc. Jpn. 2002, 71, 1832-1835.

121. Tajima, N.; Kajita, K. Experimental study organic zero-gap conductor $\alpha$-(BEDT-TTF) $)_{2} \mathrm{I}_{3}$. Sci. Technol. Adv. Mater. 2009, 10, 024308:1-024308:7.

122. Kobayashi, A.; Katayama, S.; Suzumura, Y. Theoretical study of the zero-gap organic conductor $\alpha$-(BEDT-TTF) $)_{2} \mathrm{I}_{3}$. Sci. Technol. Adv. Mater. 2009, 10, 024309:1-024309:15.

123. Iwai, S.; Yamamoto, K.; Kashiwazaki, A.; Hiramatsu, F.; Nakaya, H.; Kawakami, Y.; Yakushi, K.; Okamoto, H.; Mori, H.; Nishio, Y. Photoinduced melting of a stripe-type charge-order and metallic domain formation in a layered BEDT-TTF-based organic salt. Phys. Rev. Lett. 2007, 98, 097402:1-097402:4.

124. Yamamoto, K.; Iwai, S.; Boyko, S.; Kashiwazaki, A.; Hiramatsu, F.; Okabe, C.; Nishi, N.; Yakushi, K. Strong optical nonlinearity and its ultrafast response associated with electron ferroelectricity in an organic conductor. J. Phys. Soc. Jpn. 2008, 77, 074709:1-074709:6.

125. Bender, K.; Henning, I.; Schweitzer, D.; Dietz, K.; Endres, H.; Keller, H.J. Synthesis, structure, and physical properties of a two-dimensional organic metal, di[bis(ethylenedithiolo)tetrathio-fulvalene] triiodide, (BEDT-TTF $)_{2}{ }^{+}{ }_{3}{ }^{-}$. Mol. Cryst. Liq. Cryst. 1984, 108, 359-371.

126. Kino, H.; Fukuyama, H. Phase diagram of two-dimensional organic conductors: (BEDT-TTF) 2 X. J. Phys. Soc. Jpn. 1996, 65, 2158-2169.

127. Takahashi, T.; Hiraki, K.; Moroto, S.; Tajima, N.; Takano, Y.; Kubo, Y.; Satsukawa, H.; Chiba, R.; Yamamoto, H.M.; Kato, R.; et al. Charge disproportionation, everywhere! J. Phys. IV Fr. 2004, $114,3-8$.

128. Kakiuchi, T.; Wakabayashi, Y.; Sawa, H.; Takahashi, T.; Nakamura, T. Charge ordering in $\alpha$-(BEDT-TTF) $)_{2} \mathrm{I}_{3}$ by synchrotron x-ray diffraction. J. Phys. Soc. Jpn. 2007, 76, 113702:1-113702:4.

129. Alemany, P.; Pouget, J.-P.; Canadell, E. Essential role of anions in the charge ordering transition of $\alpha$-(BEDT-TTF $)_{2} \mathrm{I}_{3}$. Phys. Rev. B 2012, 85, 195118:1-195118:10. 
130. Yue, Y.; Yamamoto, K.; Uruichi, M.; Nakano, C.; Yakushi, K.; Yamada, S.; Hiejima, T.; Kawamoto, A. Nonuniform site-charge distribution and fluctuations of charge order in the metallic state of $\alpha$-(BEDT-TTF) ${ }_{2} \mathrm{I}_{3}$. Phys. Rev. 2010, 82, 075134:1-075134:8.

131. Yamamoto, K.; Kowalska, A.A.; Yue, Y.; Yakushi, K. Vibronic activation of molecular vibrational overtones in the infrared spectra of charge-ordered organic conductors. Phys. Rev. 2011, 84, 064306:1-064306:13.

132. Yamamoto, K.; Kowalska, A.A.; Yue, Y.; Yakushi, K. E-mv coupling of vibrational overtone in organic conductors: Relationship to optical nonlinearity and ferroelectricity. Physica B 2012, 407, $1775-1778$.

133. Ivek, T.; Korin-Hamzic, N.; Milat, O.; Tomic, S.; Clauss, C.; Drichko, N.; Schweitzer, D.; Dressel, M. Electrodynamic response of the charge ordering phase: Dielectric and optical studies of $\alpha$-(BEDT-TTF) ${ }_{2} \mathrm{I}_{3}$. Phys. Rev. 2011, 83, 165128:1-165128:13.

134. Moroto, S.; Hiraki, K.-I.; Takano, Y.; Kubo, Y.; Takahashi, T.; Yamamoto, H.M.; Nakmura, T. Charge disproportionation in the metallic state of $\alpha$-(BEDT-TTF $)_{2} \mathrm{I}_{3}$. J. Phys. IV Fr. 2004, 114, 339-340.

135. Kawai, T.; Kawamoto, A. ${ }^{13} \mathrm{C}-\mathrm{NMR}$ study of charge ordering state in the organic conductor, $\alpha$-(BEDT-TTF $)_{2} \mathrm{I}_{3}$. J. Phys. Soc. Jpn. 2009, 78, 074711:1-074711:6.

136. Yamamoto, K.; Yakushi, K. Second-Harmonic Generation Study Of Ferroelectric Organic Conductors $\alpha$-(BEDT-TTF $)_{2} \mathrm{X}\left(\mathrm{X}=\mathrm{I}_{3}\right.$ and $\left.\mathrm{I}_{2} \mathrm{Br}\right)$. Molecular Electronic and Related MaterialsControl and Probe with Light; Naito, T., Ed.; Transworld Research Network: Kerala, Indian, 2010; pp. 185-201.

137. Yamamoto, K.; Kowalska, A.A.; Yakushi, K. Direct observation of ferroelectric domains by Wigner crystallization of electrons in $\alpha$-[bis(ethylenedithio)tetetrathiafulvalene $]_{2} \mathrm{I}_{3}$. App. Phys. Lett. 2010, 96, 122901:1-122901:3.

138. Ikeda, N.; Ohsumi, H.; Ohwada, K.; Ishii, K.; Inami, T.; Kakura, K.; Murakami, Y.; Yoshii, K.; Mori, S.; Horibe, Y.; et al. Ferroelectricity from iron valence ordering in the charge-frustrated system $\mathrm{LuFe}_{2} \mathrm{O}_{4}$. Nature 2005, 436, 1136-1138.

139. Iwai, S.; Yamamoto, K.; Hiramatsu, F.; Nakaya, H.; Kawakami, Y.; Yakushi, K. Hydrostatic pressure effect on photoinduced insulator-to-metal transition in the layered organic salt $\alpha$-(BEDT-TTF) $)_{2} \mathrm{I}_{3}$. Phys. Rev. 2008, 77, 125131:1-125131:5.

140. Kawakami, Y.; Fukatsu, T.; Sakurai, Y.; Unno, H.; Itoh, H.; Iwai, S.; Sasaki, T.; Yamamoto, K.; Yakushi, K.; Yonemitsu, K. Early-State dynamics of light-matter interaction leading to the insulator-to-metal transition in a charge ordered organic crystal. Phys. Rev. Lett. 2010, 105, 246402:1-246402:4.

141. Mori, H.; Tanaka, S.; Oshima, K.; Saito, G.; Mori, T.; Maruyama, Y.; Inokuchi, H. Electrical properties and crystal structure of mercury (II) thiocyanate salts based upon BEDT-TTF with $\mathrm{Li}^{+}$, $\mathrm{K}^{+}, \mathrm{NH}_{4}{ }^{+}, \mathrm{Rb}^{+}$, and $\mathrm{Cs}^{+}$. Synth. Metal. 1991, 42, 2013-2018.

142. Mori, H.; Tanaka, S.; Oshima, M.; Saito, G.; Mori, T.; Maruyama, Y.; Inokuchi, H. Crystal and electronic structures of $(\mathrm{BEDT}-\mathrm{TTF})_{2}\left[\mathrm{MHg}(\mathrm{SCN})_{4}\right]\left(\mathrm{M}=\mathrm{K}\right.$ and $\left.\mathrm{NH}_{4}\right)$. Bull. Chem. Soc. Jpn. 1990, 63, 2183-2190. 
143. Kobayashi, H.; Kato, R.; Kobayashi, A.; Nishio, Y.; Kajita, K.; Sasaki, W. Crystal and electronic structures of layered molecular superconductor, $\theta-(\mathrm{BEDT}-\mathrm{TTF})_{2}\left(\mathrm{I}_{3}\right)_{x}\left(\mathrm{AuI}_{2}\right)_{1-x}(x<0.02)$. Chem. Lett. 1986, 833-836.

144. Tajima, N.; Tamura, M.; Nishio, Y.; Kajita, K.; Iye, Y. Transport property of an organic conductor $\alpha$-(BEDT-TTF $)_{2} \mathrm{I}_{3}$ under hydrostatic pressure-Discovery of a novel type of conductor J. Phys. Soc. Jpn. 2000, 69, 543-551.

145. Hiejima, T.; Yamada, S.; Uruichi, M.; Yakushi, K. Infrared and Raman studies of $\alpha$-(BEDT$\mathrm{TTF})_{2} \mathrm{MHg}(\mathrm{SCN})_{4}$ with $\mathrm{M}=\mathrm{NH}_{4}$ and $\mathrm{K}$ at low temperature: Breaking of inversion symmetry due to charge-ordering fluctuation. Physica B 2010, 405, 5153-5156.

146. Foury-Leylekian, P.; Ravy, S.; Pouget, J.-P.; Muller, H. X-ray study of the density wave instability of $\alpha$-(BEDT-TTF $)_{2} \mathrm{MHg}(\mathrm{SCN})_{4}$ with $\mathrm{M}=\mathrm{K}$ and Rb. Synth. Metal. 2003, 137, 1271-1272.

147. Foury-Leylekian, P.; Pouget, J.-P.; Lee, Y.-J.; Nieminen, R.M.; Ordejon, P.; Canadell, E. Density-wave instability in $\alpha$-(BEDT-TTF $)_{2} \mathrm{KHg}(\mathrm{SCN})_{4}$ studied by $\mathrm{x}$-ray diffuse scattering and by first-principle calculations. Phys. Rev. 2010, 82, 134116:1-134116:14.

148. This value was estimated from the separation of $v_{27}{ }^{j}$ mode, $\left(\sim 7 \mathrm{~cm}^{-1}\right) /\left(140 \mathrm{~cm}^{-1} / e\right)$.

149. Yamamoto, T.; Yamamoto, H.M.; Kato, R.; Uruichi, M.; Yakushi, K.; Akutsu, H.; Sato-Akutsu, A.; Kawamoto, A.; Turner, S.S.; Day, P. Inhomogeneous site charges at the boundary between the insulating, superconducting, and metallic phases of $\beta^{\prime \prime}$-type bis-ethylenedithio-tetrathiafulvalene molecular charge-transfer salts. Phys. Rev. 2008, 77, 205120:1-205120:14.

150. Bangura, A.F.; Coldea, A.I.; Singleton, J.; Ardavan, A.; Akutsu-Sato, A.; Akutsu, H.; Turner, S.S.; Day, P.; Yamamoto, T.; Yakushi, K. Robust superconducting state in the low-quasiparticle-density due to proximity to a charge-ordered state. Phys. Rev. 2005, 72, 014543:1-014543:13.

151. See ref. [46] about the reason why this equation is applicable in the range of $0 \leq \rho \leq 0.8$.

152. Although frequency is proportional to the square root of force constant, the frequency practically linearly depends upon the force constant in a narrow frequency range, $\Delta \omega=\omega(0)-\omega(1)$.

153. The $\kappa$ - and $\beta^{\prime}$-ET salts with $\rho=0.5$ have a dimer unit with large transfer integral. However, the blue shift of $v_{2}$ is not attributed to the emv (electron-molecular-vibration) coupling, because similar blue shift is found in non-dimeric $\theta-(\mathrm{ET})_{2} \mathrm{I}_{3}$ which will be shown in the next subsection.

154. Hess, L.A.; Prasad, P.N. Vibrational dephasing in organic solids: Temperature dependence of Raman active internal mode of naphthalene. J. Phys. Chem. 1980, 72, 573-579.

155. The frequency of the infrared-active $v_{27}$ mode at $\rho=0.5$ is also deviated from the linear relationship. The examination similar to $v_{2}$ is necessary including temperature dependence.

156. Kayatama, S.; Kobayashi, A.; Suzumura, Y. Effect of anion potential on the zero-gap state in the two-dimensional organic conductor $\alpha$-(BEDT-TTF $)_{2} \mathrm{I}_{3}$. J. Phys. Conf. Ser. 2008, 132, 012003:1-012003:7.

157. Kobayashi, A.; Katayama, S.; Suzumura, Y.; Fukuyama, H. Massless fermions in organic conductor. J. Phys. Soc. Jpn. 2007, 76, 034711:1-034711:6.

158. Ishibashi, S.; Tamura, T.; Kohyama, M.; Terakura, K. Ab initio electronic-structure calculation. J. Phys. Soc. Jpn. 2006, 75, 015005:1-015005:2.

159. Tamura, M.; Matsuzaki, F.; Tajima, N.; Nishio, Y.; Kajita, K. Transport study of an organic conductor, $\theta$-(BEDT-TTF) $)_{2} \mathrm{I}_{3}$. Synth. Metal. 1997, 86, 2007-2008. 
160. Tajima, N.; Tajima, A.; Tamura, M.; Kato, R.; Nishio, Y.; Kajita, K. Pressure control of transport property of organic conductors; $\alpha-, \theta$-(BEDT-TTF $)_{2} \mathrm{I}_{3}$ and $\theta$-(DIETS $)_{2}\left[\mathrm{Au}(\mathrm{CN})_{4}\right] . J$. Phys. IV Fr. 2004, 114, 263-267.

161. Miyagawa, K.; Hirayama, M.; Tamura, M.; Kanoda, K. ${ }^{13} \mathrm{C}$ NMR study on zero-gap state in the organic conductor $\theta-(\mathrm{BEDT}-\mathrm{TTF})_{2} \mathrm{I}_{3}$ under pressure. J. Phys. Soc. Jpn. 2010, 79, 063703:1-063703:3.

162. As $v_{2 \mathrm{R}}$ is mixed with $v_{3}{ }^{1}$, the hole numbers shown in the $\mathrm{CO}$ state shown in Figure $5 \mathrm{~b}, \mathrm{c}$ are inaccurate.

163. Kondo, R.; Kagoshima, S.; Tajima, N.; Kato, R. Crystal and electronic structures of the quasi-two-dimensional organic conductor $\alpha$-(BEDT-TTF $)_{2} \mathrm{I}_{3}$ and its selenium analogue $\alpha$-(BEDT-TSeF $)_{2} \mathrm{I}_{3}$ under hydrostatic pressure at room temperature. J. Phys. Soc. Jpn. 2009, 78, 114714:1-114714:7.

164. Yakushi, K.; Kanbara, H.; Tajima, H.; Kuroda, H.; Saito, G.; Mori, T. Temperature dependence of the reflectance spectra of the single crystals of bis(ethylenedithio)tetrathiafulvalene salts. $\alpha-(\mathrm{BEDT}-\mathrm{TTF})_{3}\left(\mathrm{ReO}_{4}\right)_{2}$ and $\alpha-(\mathrm{BEDT}-\mathrm{TTF})_{2} \mathrm{I}_{3}$. Bull. Chem. Soc. Jpn. 1987, 60, 4251-4257.

165. Zelezny, V.; Petzelt, J.; Swietlik, R.; Gorshunov, B.P.; Volkov, A.A.; Kozlov, G.V.; Schweitzer, D.; Keller, H.J. Far infrared response of $\alpha$ - and $\alpha_{\mathrm{t}}$-(BEDT-TTF) ${ }_{2} \mathrm{I}_{3}$. J. Phys. Fr. 1990, 51, 869-881.

166. Dressel, M.; Gruner, G.; Pouget, J.P.; Breining, A.; Schweitzer, D. Field and frequency dependent transport in the two-dimensional organic conductor $\alpha$-(BEDT-TTF) $)_{2} \mathrm{I}_{3}$. J. Phys. I Fr. 1994, 4, 579-594.

167. Clauss, C.; Drichko, N.; Schweitzer, D.; Dressel, M. Charge-order gap in $\alpha-(B E D T-T T F){ }_{2} \mathrm{I}_{3}$. Physica B 2010, 405, S144-S146.

168. Tamura, K.; Ozawa, T.; Bando, Y.; Kawamoto, T.; Mori, T. Voltage oscillation associated with nonlinear conductivity in the organic conductor $\alpha$-(BEDT-TTF $)_{2} \mathrm{I}_{3}$. J. Appl. Phys. 2010, 107, 103716:1-103716:5.

169. Ivek, T.; Korin-Hamzic, B.; Milat, O.; Tomic, S.; Clauss, C.; Drichko, N.; Schweitzer, D.; Dressel, M. Collective excitation in the charge-ordered phase of $\alpha$-(BEDT-TTF $)_{2} \mathrm{I}_{3}$. Phys. Rev. Lett. 2010, 104, 206406:1-206406:4.

170. Dressel, M.; Drichko, N.; Kaiser, S. Collective charge-order excitations. Physica C 2010, 470, S589-S591.

171. Watanabe, M.; Nishikawa, M.; Nogami, Y.; Oshima, K.; Saito, G. Low temperature structure of the $\alpha^{\prime}-(\mathrm{BEDT}-\mathrm{TTF})_{2} \mathrm{IBr}_{2}$ organic magnetic semiconductor. J. Korean Phys. Soc. 1997, 31, 95-98.

172. Tokumoto, M.; Anzai, H.; Ishiguro, T.; Saito, G.; Kobayashi, H.; Kato, R.; Kobayashi, A. Electrical and magnetic properties of organic semiconductors, (BEDT-TTF) ${ }_{2} \mathrm{X}\left(\mathrm{X}=\mathrm{IBr}_{2}, \mathrm{IBrCl}\right.$, and $\mathrm{ICl}_{2}$ ). Synth. Metal. 1987, 19, 215-220.

173. Maniwa, Y.; Takahashi, T.; Saito, G. ${ }^{1} \mathrm{H}$ NMR in organic superconductor $\beta$-(BEDT-TTF) ${ }_{2} \mathrm{I}_{3}$. J. Phys. Soc. Jpn. 1986, 55, 47-50.

174. Nogami, Y.; Kagoshima, S.; Sugano, T.; Saito, G. X-ray evidence for structural changes in the organic conductors, $\alpha-(\mathrm{BEDT}-\mathrm{TTF})_{2} \mathrm{I}_{3}, \alpha-(\mathrm{BEDT}-\mathrm{TTF})_{2} \mathrm{IBr}_{2}$, and $\beta-(\mathrm{BEDT}-\mathrm{TTF})_{2} \mathrm{I}_{3}$. Synth. Metal. 1986, 16, 367-377.

175. Inokuchi, M.; Yakushi, K.; Kinoshita, M.; Saito, G. Optical properties of $\alpha^{\prime}-(\mathrm{BEDT}-\mathrm{TTF})_{2} \mathrm{IBr}_{2}$. Synth. Metal. 1992, 103, 2102-2103. 
176. Yue, Y.; Nakano, C.; Yamamoto, K.; Uruichi, M.; Yakushi, K.; Kawamoto, A. Charge-ordering phase transition in $\alpha^{\prime}-(B E D T-T T F)_{2} \mathrm{IBr}_{2}$. J. Phys. Conf. Ser. 2008, 132, 012007:1-012007:7.

177. Yue, Y.; Yamamoto, K.; Nakano, C.; Uruichi, M.; Yakushi, K.; Inokuchi, M.; Hiejima, T.; Kawamoto, A. Order-disorder type of charge-ordering phase transition in narrow-bandwidth compound, $\alpha^{\prime}$-(BEDT-TTF) ${ }_{2} \mathrm{IBr}_{2}$. Physica B 2010, 405, S232-S236.

178. Kowalska, A.A.; Yamamoto, K.; Yakushi, K. Ferroelectric polarization in $\alpha^{\prime}-(\mathrm{ET})_{2} \mathrm{IBr}_{2}$ studied by second-harmonic generation microscopy. J. Phys. Conf. Ser. 2008, 132, 012006:1-012006:5.

179. Yamashita, A.; Watanabe, M.; Kobayashi, K.; Kumai, R.; Yamamoto, K.; Yakushi, K.; Noda, Y. SR-based study of successive phase transition of $\alpha^{\prime}$-(BEDT-TTF $)_{2} \mathrm{IBr}_{2}$ II. Meeting Abstr. Phys. Soc. Jpn. 2012, 67, 907.

180. Sugano, T.; Saito, G.; Kinoshita, M. Conduction-electron-spin resonance in organic conductors: $\mathrm{A}$ and $\mathrm{b}$ phases of di[bis(ethylenedithiolo)tetrathiafulvalene]triiodide [(BEDT-TTF $\left.)_{2} \mathrm{I}_{3}\right]$. Phys. Rev. 1986, 34, 117-125.

181. The optical conductivities from $50-6000 \mathrm{~cm}^{-1}$ of $\alpha^{\prime}-\mathrm{IBr}_{2}, \alpha-\mathrm{I}_{3}$ and $\alpha-\mathrm{NH}_{4} \mathrm{Hg}$ were obtained in our research group. The low-frequency region from $0-600 \mathrm{~cm}^{-1}$ of $\beta^{\prime \prime}$-(BEDT-TTF)(TCNQ), $\theta_{\mathrm{m}}-\mathrm{RbZn}, \theta_{\mathrm{o}}-\mathrm{RbZn}$, and $\theta-\mathrm{CsZn}$ were extrapolated to the de conductivity value by straight line. The optical conductivities of $\theta-\mathrm{RbZn}$ and $\theta-\mathrm{I}_{3}$ were taken from the papers reported by Wang [95] and Takenaka [112], respectively.

182. Van der Marel, D. Optical Signatures of Electron Correlations in the Cuprates. In Strong Interactions in Low Dimensions; Baeriswyl, D., Degiorgi, L., Eds.; Kluwer Academic Publishers: Dordrecht, The Netherlands, 2004; pp. 237-276.

(C) 2012 by the authors; licensee MDPI, Basel, Switzerland. This article is an open access article distributed under the terms and conditions of the Creative Commons Attribution license (http://creativecommons.org/licenses/by/3.0/). 